\title{
Characterization of nuclear effects in muon-neutrino scattering on hydrocarbon with a measurement of final-state kinematics and correlations in charged-current pionless interactions at T2K
}

K. Abe,${ }^{46}$ J. Amey, ${ }^{16}$ C. Andreopoulos, ${ }^{44,26}$ L. Anthony, ${ }^{26}$ M. Antonova, ${ }^{15}$ S. Aoki,${ }^{23}$ A. Ariga, ${ }^{2}$ Y. Ashida,${ }^{24}$ Y. Azuma, ${ }^{33}$ S. Ban, ${ }^{24}$ M. Barbi,${ }^{38}$ G. J. Barker, ${ }^{55}$ G. Barr, ${ }^{34}$ C. Barry,${ }^{26}$ M. Batkiewicz, ${ }^{12}$ V. Berardi, ${ }^{17}$ S. Berkman, ${ }^{4,51}$ R. M. Berner, ${ }^{2}$ L. Berns, ${ }^{48}$ S. Bhadra, ${ }^{5}$ S. Bienstock, ${ }^{35}$ A. Blondel, ${ }^{11}$ S. Bolognesi, ${ }^{6}$ S. Bordoni, ${ }^{14,{ }^{*}}$ B. Bourguille, ${ }^{14}$ S. B. Boyd, ${ }^{55}$ D. Brailsford, ${ }^{25}$ A. Bravar, ${ }^{11}$ C. Bronner, ${ }^{46}$ M. Buizza Avanzini, ${ }^{10}$ J. Calcutt, ${ }^{28}$ T. Campbell, ${ }^{8}$ S. Cao, ${ }^{13}$

S. L. Cartwright, ${ }^{42}$ M. G. Catanesi, ${ }^{17}$ A. Cervera, ${ }^{15}$ A. Chappell, ${ }^{55}$ C. Checchia, ${ }^{19}$ D. Cherdack, ${ }^{8}$ N. Chikuma, ${ }^{45}$ G. Christodoulou, ${ }^{26}$ J. Coleman, ${ }^{26}$ G. Collazuol, ${ }^{19}$ D. Coplowe, ${ }^{34}$ A. Cudd ${ }^{28}$ A. Dabrowska, ${ }^{12}$ G. De Rosa ${ }^{18}$ T. Dealtry, ${ }^{25}$ P. F. Denner, ${ }^{55}$ S. R. Dennis, ${ }^{26}$ C. Densham, ${ }^{44}$ F. Di Lodovico, ${ }^{37}$ S. Dolan, ${ }^{10,6}$ O. Drapier, ${ }^{10}$ K. E. Duffy,${ }^{34}$ J. Dumarchez,${ }^{35}$ P. Dunne, ${ }^{16}$ S. Emery-Schrenk, ${ }^{6}$ A. Ereditato, ${ }^{2}$ T. Feusels, ${ }^{4,51}$ A. J. Finch, ${ }^{25}$ G. A. Fiorentini, ${ }^{59}$ G. Fiorillo, ${ }^{18}$ C. Francois, ${ }^{2}$ M. Friend, ${ }^{13, \dagger}$ Y. Fujii, ${ }^{13, \dagger}$ D. Fukuda ${ }^{32}$ Y. Fukuda, ${ }^{29}$ A. Garcia, ${ }^{14}$ C. Giganti, ${ }^{35}$ F. Gizzarelli, ${ }^{6}$ T. Golan ${ }^{57}$ M. Gonin, ${ }^{10}$ D. R. Hadley, ${ }^{55}$ L. Haegel, ${ }^{11}$ J. T. Haigh, ${ }^{55}$ P. Hamacher-Baumann, ${ }^{41}$ D. Hansen, ${ }^{36}$ J. Harada ${ }^{33}$ M. Hartz, ${ }^{22,51}$ T. Hasegawa, ${ }^{13 \dagger}$ N. C. Hastings, ${ }^{38}$ T. Hayashino, ${ }^{24}$ Y. Hayato, ${ }^{46,22}$ T. Hiraki ${ }^{24}$ A. Hiramoto, ${ }^{24}$ S. Hirota ${ }^{24}$ M. Hogan, ${ }^{8}$ J. Holeczek, ${ }^{43}$ F. Hosomi ${ }_{45}$ A. K. Ichikawa, ${ }^{24}$ M. Ikeda, ${ }^{46}$ J. Imber, ${ }^{10}$ T. Inoue,${ }^{33}$ R. A. Intonti, ${ }^{17}$ T. Ishida, ${ }^{13,}$,

T. Ishii, ${ }^{13, \dagger}$ K. Iwamoto, ${ }^{45}$ A. Izmaylov, ${ }^{15,21}$ B. Jamieson, ${ }^{56}$ M. Jiang, ${ }^{24}$ S. Johnson, ${ }^{7}$ P. Jonsson, ${ }^{16}$ C. K. Jung, ${ }^{31,}$ M. Kabirnezhad ${ }^{30}$ A. C. Kaboth, ${ }^{40,44}$ T. Kajita, ${ }^{47, *}$ H. Kakuno, ${ }^{49}$ J. Kameda, ${ }^{46}$ D. Karlen, ${ }^{52,51}$ T. Katori ${ }^{37}$ E. Kearns, ${ }^{3,22, \$}$ M. Khabibullin, ${ }^{21}$ A. Khotjantsev, ${ }^{21}$ H. Kim, ${ }^{33}$ J. Kim, ${ }_{4,51}$ S. King, ${ }^{37}$ J. Kisiel, ${ }^{43}$ A. Knight, ${ }^{55}$ A. Knox, ${ }^{25}$ T. Kobayashi, ${ }^{13,+}$ L. Koch, ${ }^{41}$ T. Koga ${ }^{45}$ P. P. Koller, ${ }^{2}$ A. Konaka, ${ }^{51}$ L. L. Kormos, ${ }^{25}$ Y. Koshio, ${ }^{32,}$ K. Kowalik, ${ }^{30}$ Y. Kudenko ${ }^{21, \S}$ R. Kurjata ${ }^{54}$ T. Kutter, ${ }^{27}$ L. Labarga, ${ }^{1}$ J. Lagoda ${ }^{30}$ I. Lamont, ${ }^{25}$ M. Lamoureux, ${ }^{6}$ P. Lasorak, ${ }^{37}$ M. Laveder, ${ }^{19}$ M. Lawe, ${ }^{25}$ M. Licciardi ${ }^{10}$ T. Lindner ${ }^{51}$ Z. J. Liptak, ${ }^{7}$ R. P. Litchfield, ${ }^{16}$ X. Li ${ }^{31}$ A. Longhin, ${ }^{19}$ J. P. Lopez, ${ }^{7}$ T. Lou, ${ }^{45}$ L. Ludovici, ${ }^{20}$ X. Lu, ${ }^{34}$ L. Magaletti, ${ }^{17}$ K. Mahn, ${ }^{28}$ M. Malek, ${ }^{42}$ S. Manly, ${ }^{39}$ L. Maret,${ }^{11}$ A. D. Marino, ${ }^{7}$ J. F. Martin, ${ }^{50}$ P. Martins, ${ }_{59}^{37}$ S. Martynenko, ${ }^{31}$ T. Maruyama, ${ }^{13, \dagger}$ V. Matveev ${ }^{21}$ K. Mavrokoridis, ${ }^{26}$ W. Y. Ma, ${ }^{16}$ E. Mazzucato, ${ }^{6}$ M. McCarthy, ${ }^{59}$ N. McCauley, ${ }^{26}$ K. S. McFarland, ${ }^{39}$ C. McGrew, ${ }^{31}$ A. Mefodiev, ${ }^{21}$ C. Metelko, ${ }^{26}$ M. Mezzetto, ${ }^{19}$ A. Minamino, ${ }^{58}$ O. Mineev, ${ }^{21}$ S. Mine, ${ }^{5}$ A. Missert, ${ }^{7}$ M. Miura, ${ }^{46, \$}$ S. Moriyama, ${ }^{46, \$}$ J. Morrison, ${ }^{28}$ Th. A. Mueller, ${ }^{10}$ Y. Nagai, ${ }^{7}$ T. Nakadaira ${ }^{13, \uparrow}$ M. Nakahata, ${ }^{46,22}$ K. G. Nakamura, ${ }^{24}$ K. Nakamura, ${ }^{22,13, \dagger}$ K. D. Nakamura, ${ }^{24}$ Y. Nakanishi, ${ }^{24}$ S. Nakayama ${ }^{46, \$}$ T. Nakaya,${ }^{24,22}$ K. Nakayoshi, ${ }^{13, \uparrow}$ C. Nantais, ${ }^{50}$ C. Nielsen, ${ }^{4,51}$ K. Niewczas, ${ }^{57}$ K. Nishikawa, ${ }^{13, \dagger}$ Y. Nishimura, ${ }^{47}$ P. Novella, ${ }^{15}$ J. Nowak ${ }^{25}$ H. M. O'Keeffe, ${ }^{25}$ K. Okumura, ${ }^{47,22}$ T. Okusawa, ${ }^{33}$ W. Oryszczak, ${ }^{53}$ S. M. Oser, ${ }^{4,51}$ T. Ovsyannikova, ${ }^{21}$ R. A. Owen, ${ }^{37}$ Y. Oyama, ${ }^{13, \uparrow}$ V. Palladino, ${ }^{18}$ J. L. Palomino, ${ }^{31}$ V. Paolone, ${ }^{36}$ P. Paudyal, ${ }^{26}$ M. Pavin,${ }^{51}$ D. Payne, ${ }^{26}$ Y. Petrov, ${ }^{4,51}$

L. Pickering, ${ }^{28}$ E. S. Pinzon Guerra, ${ }^{59}$ C. Pistillo, ${ }^{2}$ B. Popov, ${ }^{35,}$ M. Posiadala-Zezula, ${ }^{53}$ A. Pritchard, ${ }^{26}$ P. Przewlocki ${ }^{30}$ B. Quilain, ${ }^{22}$ T. Radermacher, ${ }^{41}$ E. Radicioni, ${ }^{17}$ P. N. Ratoff, ${ }^{25}$ M. A. Rayner, ${ }^{11}$ E. Reinherz-Aronis, ${ }^{8}$ C. Riccio, ${ }^{18}$ E. Rondio, ${ }^{30}$ B. Rossi, ${ }^{18}$ S. Roth, ${ }^{41}$ A. C. Ruggeri, ${ }^{18}$ A. Rychter, ${ }^{54}$ K. Sakashita, ${ }^{13, \dagger}$ F. Sánchez, ${ }^{14}$ S. Sasaki, ${ }^{49}$

E. Scantamburlo, ${ }^{11}$ K. Scholberg, ${ }^{9, \$}$ J. Schwehr, ${ }^{8}$ M. Scott, ${ }^{51}$ Y. Seiya ${ }^{33}$ T. Sekiguchi, ${ }^{13, \dagger}$ H. Sekiya, ${ }^{46,22, \downarrow}$ D. Sgalaberna ${ }^{11}$ R. Shah, ${ }^{44,34}$ A. Shaikhiev, ${ }^{21}$ F. Shaker, ${ }^{56}$ D. Shaw, ${ }^{25}$ M. Shiozawa, ${ }^{46,22}$ A. Smirnov, ${ }^{21}$ M. Smy, J. T. Sobczyk ${ }^{57}$ H. Sobel, ${ }^{5,22}$ J. Steinmann, ${ }^{41}$ T. Stewart, ${ }^{44}$ P. Stowell, ${ }^{42}$ Y. Suda ${ }^{45}$ S. Suvorov, ${ }^{21,6}$ A. Suzuki, ${ }^{23}$ S. Y. Suzuki, ${ }^{1, \dagger}$

Y. Suzuki, ${ }^{22}$ R. Tacik, ${ }^{38,51}$ M. Tada, ${ }^{13, \dagger}$ A. Takeda, ${ }^{46}$ Y. Takeuchi, ${ }^{23,22}$ R. Tamura, ${ }^{45}$ H. K. Tanaka, ${ }^{46, \$}$ H. A. Tanaka, ${ }^{50,51,{ }^{* * *}}$ T. Thakore, ${ }^{27}$ L. F. Thompson, ${ }^{42}$ W. Toki ${ }^{8}$ T. Tsukamoto, ${ }^{13, \dagger}$ M. Tzanov ${ }^{27}$ W. Uno, ${ }^{24}$ M. Vagins,${ }^{22,5}$ Z. Vallari ${ }^{31}$ G. Vasseur, ${ }^{6}$ C. Vilela, ${ }^{31}$ T. Vladisavljevic, ${ }^{34,22}$ T. Wachala, ${ }^{12}$ J. Walker, ${ }^{56}$ C. W. Walter, ${ }^{9,5}$ Y. Wang,${ }^{31}$ D. Wark, ${ }^{44,34}$ M. O. Wascko, ${ }^{16}$ A. Weber, ${ }^{44,34}$ R. Wendell, ${ }^{24, \$}$ M. J. Wilking, ${ }^{31}$ C. Wilkinson, ${ }^{2}$ J. R. Wilson, ${ }^{37}$ R. J. Wilson, ${ }^{8}$ C. Wret, ${ }^{16}$ Y. Yamada,${ }^{13, \dagger}$ K. Yamamoto, ${ }^{33}$ S. Yamasu, ${ }^{32}$ C. Yanagisawa, ${ }^{31, \dagger \dagger}$ T. Yano, ${ }^{46}$ S. Yen, ${ }^{51}$ N. Yershov, ${ }^{21}$ M. Yokoyama, ${ }^{45, \$}$ M. Yu, ${ }^{59}$ A. Zalewska, ${ }^{12}$ J. Zalipska, ${ }^{30}$ L. Zambelli, ${ }^{13, \dagger}$ K. Zaremba, ${ }^{54}$ M. Ziembicki ${ }^{54}$ E. D. Zimmerman, ${ }^{7}$ M. Zito, ${ }^{6}$ S. Zsoldos, ${ }^{37}$ and A. Zykova ${ }^{21}$

(The T2K Collaboration)

\author{
${ }^{1}$ University Autonoma Madrid, Department of Theoretical Physics, 28049 Madrid, Spain \\ ${ }^{2}$ University of Bern, Albert Einstein Center for Fundamental Physics, Laboratory for High Energy Physics \\ (LHEP), Bern, Switzerland \\ ${ }^{3}$ Boston University, Department of Physics, Boston, Massachusetts, USA \\ ${ }^{4}$ University of British Columbia, Department of Physics and Astronomy, \\ Vancouver, British Columbia, Canada
}


${ }^{5}$ University of California, Irvine, Department of Physics and Astronomy, Irvine, California, USA ${ }^{6}$ IRFU, CEA Saclay, Gif-sur-Yvette, France

${ }^{7}$ University of Colorado at Boulder, Department of Physics, Boulder, Colorado, USA

${ }^{8}$ Colorado State University, Department of Physics, Fort Collins, Colorado, USA

${ }^{9}$ Duke University, Department of Physics, Durham, North Carolina, USA

${ }^{10}$ Ecole Polytechnique, IN2P3-CNRS, Laboratoire Leprince-Ringuet, Palaiseau, France

${ }^{11}$ University of Geneva, Section de Physique, DPNC, Geneva, Switzerland

${ }^{12}$ H. Niewodniczanski Institute of Nuclear Physics PAN, Cracow, Poland

${ }^{13}$ High Energy Accelerator Research Organization (KEK), Tsukuba, Ibaraki, Japan

${ }^{14}$ Institut de Fisica d'Altes Energies (IFAE), The Barcelona Institute of Science and Technology, Campus $U A B$, Bellaterra (Barcelona) Spain

${ }^{15}$ IFIC (CSIC \& University of Valencia), Valencia, Spain

${ }^{16}$ Imperial College London, Department of Physics, London, United Kingdom

${ }^{17}$ INFN Sezione di Bari and Università e Politecnico di Bari, Dipartimento Interuniversitario di Fisica, Bari, Italy

${ }^{18}$ INFN Sezione di Napoli and Università di Napoli, Dipartimento di Fisica, Napoli, Italy

${ }^{19}$ INFN Sezione di Padova and Università di Padova, Dipartimento di Fisica, Padova, Italy

${ }^{20}$ INFN Sezione di Roma and Università di Roma "La Sapienza”, Roma, Italy

${ }^{21}$ Institute for Nuclear Research of the Russian Academy of Sciences, Moscow, Russia

${ }^{22}$ Kavli Institute for the Physics and Mathematics of the Universe (WPI),

The University of Tokyo Institutes for Advanced Study, University of Tokyo, Kashiwa, Chiba, Japan

${ }^{23}$ Kobe University, Kobe, Japan

${ }^{24}$ Kyoto University, Department of Physics, Kyoto, Japan

${ }^{25}$ Lancaster University, Physics Department, Lancaster, United Kingdom

${ }^{26}$ University of Liverpool, Department of Physics, Liverpool, United Kingdom

${ }^{27}$ Louisiana State University, Department of Physics and Astronomy, Baton Rouge, Louisiana, USA

${ }^{28}$ Michigan State University, Department of Physics and Astronomy, East Lansing, Michigan, USA

${ }^{29}$ Miyagi University of Education, Department of Physics, Sendai, Japan

${ }^{30}$ National Centre for Nuclear Research, Warsaw, Poland

${ }^{31}$ State University of New York at Stony Brook, Department of Physics and Astronomy, Stony Brook, New York, USA

${ }^{32}$ Okayama University, Department of Physics, Okayama, Japan

${ }^{33}$ Osaka City University, Department of Physics, Osaka, Japan

${ }^{34}$ Oxford University, Department of Physics, Oxford, United Kingdom

${ }^{35}$ UPMC, Université Paris Diderot, CNRS/IN2P3, Laboratoire de Physique Nucléaire et de Hautes Energies (LPNHE), Paris, France

${ }^{36}$ University of Pittsburgh, Department of Physics and Astronomy, Pittsburgh, Pennsylvania, USA

${ }^{37}$ Queen Mary University of London, School of Physics and Astronomy, London, United Kingdom

${ }^{38}$ University of Regina, Department of Physics, Regina, Saskatchewan, Canada

${ }^{39}$ University of Rochester, Department of Physics and Astronomy, Rochester, New York, USA

${ }^{40}$ Royal Holloway University of London, Department of Physics, Egham, Surrey, United Kingdom

${ }^{41}$ RWTH Aachen University, III. Physikalisches Institut, Aachen, Germany

${ }^{42}$ University of Sheffield, Department of Physics and Astronomy, Sheffield, United Kingdom

${ }^{43}$ University of Silesia, Institute of Physics, Katowice, Poland

${ }^{44}$ STFC, Rutherford Appleton Laboratory, Harwell Oxford, and Daresbury Laboratory,

Warrington, United Kingdom

${ }^{45}$ University of Tokyo, Department of Physics, Tokyo, Japan

${ }^{46}$ University of Tokyo, Institute for Cosmic Ray Research, Kamioka Observatory, Kamioka, Japan

${ }^{47}$ University of Tokyo, Institute for Cosmic Ray Research,

Research Center for Cosmic Neutrinos, Kashiwa, Japan

${ }^{48}$ Tokyo Institute of Technology, Department of Physics, Tokyo, Japan

${ }^{49}$ Tokyo Metropolitan University, Department of Physics, Tokyo, Japan

${ }^{50}$ University of Toronto, Department of Physics, Toronto, Ontario, Canada

${ }^{51}$ TRIUMF, Vancouver, British Columbia, Canada

${ }^{52}$ University of Victoria, Department of Physics and Astronomy, Victoria, British Columbia, Canada

${ }^{53}$ University of Warsaw, Faculty of Physics, Warsaw, Poland

${ }^{54}$ Warsaw University of Technology, Institute of Radioelectronics, Warsaw, Poland

${ }^{55}$ University of Warwick, Department of Physics, Coventry, United Kingdom

${ }^{56}$ University of Winnipeg, Department of Physics, Winnipeg, Manitoba, Canada

${ }^{57}$ Wroclaw University, Faculty of Physics and Astronomy, Wroclaw, Poland 


\author{
${ }^{58}$ Yokohama National University, Faculty of Engineering, Yokohama, Japan \\ ${ }^{59}$ York University, Department of Physics and Astronomy, Toronto, Ontario, Canada
}

(Received 3 April 2018; published 9 August 2018)

\begin{abstract}
This paper reports measurements of final-state proton multiplicity, muon and proton kinematics, and their correlations in charged-current pionless neutrino interactions, measured by the T2K ND280 near detector in its plastic scintillator $\left(\mathrm{C}_{8} \mathrm{H}_{8}\right)$ target. The data were taken between years 2010 and 2013, corresponding to approximately $6 \times 10^{20}$ protons on target. Thanks to their exploration of the proton kinematics and of imbalances between the proton and muon kinematics, the results offer a novel probe of the nuclear-medium effects most pertinent to the (sub-) $\mathrm{GeV}$ neutrino-nucleus interactions that are used in accelerator-based long-baseline neutrino oscillation measurements. These results are compared to many neutrino-nucleus interaction models which all fail to describe at least part of the observed phase space. In case of events without a proton above a detection threshold in the final state, a fully consistent implementation of the local Fermi gas model with multinucleon interactions gives the best description of the data. In the case of at least one proton in the final state, the spectral function model agrees well with the data, most notably when measuring the kinematic imbalance between the muon and the proton in the plane transverse to the incoming neutrino. Within the models considered, only the existence of multinucleon interactions are able to describe the extracted cross section within regions of high transverse kinematic imbalance. The effect of final-state interactions is also discussed.
\end{abstract}

DOI: 10.1103/PhysRevD.98.032003

\section{INTRODUCTION}

Neutrino interactions with nuclei are the experimental tool exploited to provide evidence of neutrino oscillations [1-8] and to search for leptonic $C P$-symmetry violation [9-12]. In long-baseline accelerator-based neutrino oscillation experiments, neutrino beams are produced with energies in the range of hundreds of $\mathrm{MeV}$ to a few $\mathrm{GeV}$. The produced neutrinos interact then with the bound nucleons of nuclei in the detectors via reactions such as quasielastic scattering (QE), resonant production (RES), and deep inelastic scattering (DIS). A precise measurement of the oscillation parameters relies on the understanding of the incoming neutrino beam flux, of the scattering of neutrinos with nucleons, and of the nuclear medium effects in the nucleus. The systematic uncertainties arising from neutrino-nucleus interactions, especially those related to nuclear effects, are currently one of the limiting factors for

\footnotetext{
*Present address: CERN, CH-1211 Geneva 23, Switzerland. Also at J-PARC, Tokai, Japan.

${ }^{*}$ Affiliated member at Kavli IPMU (WPI), the University of Tokyo, Japan.

§Also at National Research Nuclear University "MEPhI" and Moscow Institute of Physics and Technology, Moscow, Russia. "Also at JINR, Dubna, Russia.

*** Also at Institute of Particle Physics, Canada.

Also at BMCC/CUNY, Science Department, New York, New York, USA.

Published by the American Physical Society under the terms of the Creative Commons Attribution 4.0 International license. Further distribution of this work must maintain attribution to the author(s) and the published article's title, journal citation, and DOI. Funded by SCOAP ${ }^{3}$.
}

oscillation measurements [13] in T2K [14] and NOvA [15], and will become the dominant uncertainties for future long-baseline experiments, such as DUNE [12] and HyperKamiokande [16].

Neutrinos of such energies can probe nuclear structure at the nucleon level and therefore an accurate description of the nucleus in terms of nucleonic degrees of freedom is essential. To a first approximation, in the independent particle model (IPM), each nucleon is subject to Fermi motion (FM) and a mean-field potential. It is then common to factorize neutrino-nucleus interactions into an interaction with such a bound nucleon (the impulse approximation), leaving the remaining nucleus in a one-particle-one-hole $(1 \mathrm{p} 1 \mathrm{~h})$ excitation state, and a separate description of the subsequent final state reinteractions inside the nucleus [17]. Driven by precision measurements of electron-nucleus scattering and first large statistics neutrino-nucleus scattering measurements $[18,19]$, various theoretical developments beyond these approximations have been proposed. In the random phase approximation (RPA) approach [20-24], collective excitations approximated as a superposition of $1 \mathrm{p} 1 \mathrm{~h}$ excitations are calculated. This particular medium effect is parametrized as a correction factor to the interaction cross section as a function of the squared fourmomentum transfer $Q^{2}$. In addition to such long-range correlations, short-range correlations (SRCs) are also captured by the spectral function (SF) approach [25-28], which accounts for nucleon-nucleon correlations beyond the mean-field dynamics. These correlations produce an enhancement in the ground-state nucleon momentum distribution beyond the Fermi momentum, and can lead to two-particle-two-hole (2p2h) excitations of the nucleus 
(and, more in general, to npnh excitations with $\mathrm{n}>1$ ). Formalisms developed for electron-nucleus scattering have been adapted to describe neutrino data, proposing that $2 \mathrm{p} 2 \mathrm{~h}$ contributions, notably due to meson-exchange currents (MEC), might be significant in neutrino-nucleus interactions [24,29-34].

Among the reactions relevant for $\mathrm{GeV}$ energy neutrinos, the charged-current $(\mathrm{CC}) \mathrm{QE}$,

$$
\iota \mathrm{N} \rightarrow \ell \mathrm{N}^{\prime},
$$

is of primary importance for neutrino detection in oscillation experiments, where $\nu$ and $\ell$ are the neutrino and the corresponding charged lepton, $\mathrm{N}$ and $\mathrm{N}^{\prime}$ are the initial-and final-state nucleons. Embedded in a nucleus, the final-state nucleon propagates through and interacts with the nucleus remnant. These final-state interactions (FSI) could be highly inelastic, causing energy dissipation which can prevent hadrons escaping the nuclear medium or alternatively stimulate additional hadrons to be emitted. As a result, the QE reaction in Eq. (1) is not directly accessible. What can be measured are the $\mathrm{CC}$ interactions without pion in the extra-nucleus final state $(\mathrm{CC} 0 \pi)$. This process includes not only other reactions such as pion production, in which the pion is absorbed inside the nucleus, but also $2 \mathrm{p} 2 \mathrm{~h}$ excitations involving two-nucleon knockout. $\mathrm{CC} 0 \pi$ (sometimes called "CCQE-like") interactions have been extensively measured [19,35-45], yet the unambiguous identification of various nuclear effects has proved difficult. This is primarily because the often measured single-particle final-state kinematics, such as momentum and angular distributions, are determined by both the intrinsic dynamics of Eq. (1) and by nuclear effects.

This paper reports measurements of muon-neutrino $\mathrm{CC} 0 \pi$ interactions with the $\mathrm{T} 2 \mathrm{~K}$ beam, which has a peak energy of around $600 \mathrm{MeV}$. The multidifferential cross section using muon and proton kinematics, their correlations, and the final-state multiplicity of protons (above a threshold energy) are measured. These measurements are performed using the $\mathrm{T} 2 \mathrm{~K}$ near detector (ND280), on a plastic scintillator $\left(\mathrm{C}_{8} \mathrm{H}_{8}\right)$ target, with approximately $6 \times 10^{20}$ protons on target (POT). The main aim of such new measurements is to improve the understanding of nuclear effects in neutrino interactions, notably with a view to minimizing the corresponding uncertainties in neutrino oscillation measurements. In oscillation measurements neutrino-interaction models are used to infer the neutrino energy from the final state particles and to extrapolate the near detector constraints to the far detector. To test the correctness of such inference, detailed comparisons of the measured cross sections with the most recent neutrino-nucleus interaction models are reported in this paper.

The modeling of neutrino energy reconstruction in the $\mathrm{CC} 0 \pi$ sample, exploited for neutrino oscillation measurements, is affected by large uncertainties due to nuclear effects: even when protons can be in principle detected, the detector response depends on the actual kinematics of the outgoing protons. In the absence of a robust model prediction on the hadronic final state, a multidifferential measurement of single-particle kinematics and nucleon multiplicity, provides valuable input for the modeling of neutrino energy reconstruction and detector response. Furthermore, measurements of proton kinematics from neutrino-nucleus scattering may be used to infer neutron multiplicity and kinematics in the corresponding antineutrino reaction. While the single-particle kinematics and the multiplicity measurements provide a comprehensive description of the $\mathrm{CC} 0 \pi$ final state, the measurement of muon-proton correlations in the final state provides a powerful probe of nuclear effects. Considering the dynamics of Eq. (1) in the case of scattering on a free nucleon $\nu \mathrm{n} \rightarrow \ell \mathrm{p}$ in the absence of nuclear effects, the final-state proton kinematics can be uniquely determined by that of the muon. In a $\mathrm{CC} 0 \pi$ measurement the deviation of proton multiplicity and kinematics from what is expected in the simple process of Eq. (1) originates solely from nuclear effects. Such deviations can be characterized using socalled transverse kinematic imbalances (introduced for the first time in Ref. [46]) and proton inferred kinematics, which are measured in the analyses presented here.

This paper is organized as follows. After a short description of the T2K experiment in Sec. II, the measurements presented in this paper and the new variables are introduced in Sec. III. Section IV describes the analysis procedure, including the simulations used, the event selection and the method for cross-section evaluation. Following this the results are reported for each of three analyses: one using proton and muon kinematics, another using transverse kinematic imbalances and a third using proton inferred kinematics. The interpretation of the results is discussed in Sec. V, followed by conclusions in Sec. VI.

\section{THE T2K EXPERIMENT}

The Tokai-to-Kamioka (T2K) experiment [14] is an accelerator-based long-baseline experiment which measures neutrino oscillations in a $\nu_{\mu}\left(\bar{\nu}_{\mu}\right)$ beam [9]. The T2K neutrino beam is produced by the Japan Proton Accelerator Research Complex. A $30 \mathrm{GeV}$ proton beam collides with a graphite target producing positive and negative pions and kaons which are focused and charge-selected by three horn magnets. The positive (negative) hadrons decay to produce a flux highly dominated by $\nu_{\mu}\left(\bar{\nu}_{\mu}\right)$ [47].

The Super-Kamiokande far detector is located $295 \mathrm{~km}$ away from the production point and sits $2.5^{\circ}$ off the beam axis. T2K is further equipped with two near detectors: ND280 and INGRID. INGRID [48] is designed to monitor the direction of the neutrino beam whilst ND280 is dedicated to the study of the un-oscillated spectrum of neutrinos at $280 \mathrm{~m}$ from the production target and is the detector used by the analyses presented here. ND280 is positioned off-axis so that it has the same peak neutrino 


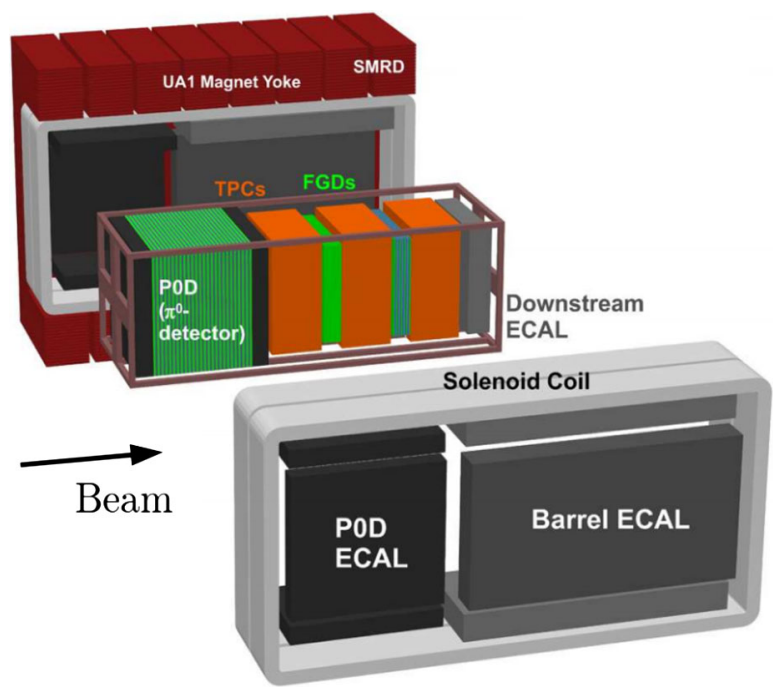

FIG. 1. An exploded view of the ND280 off-axis near detector labeling each sub-detector. Adapted from [14].

energy as Super-Kamiokande. Such configuration ensures a narrow energy spectrum of the beam centered around $600 \mathrm{MeV}$, in correspondence with the oscillation maximum. It also suppresses the intrinsic $\nu_{e}$ and the non-QE interactions, which are primarily produced by the highenergy tail of the neutrino flux. ND280 is composed of an upstream $\pi^{0}$ detector (POD) [49] and a central tracker region, described below, surrounded by an electromagnetic calorimeter (ECal) [50], consisting of interleaved layers of lead and scintillator, which itself is all contained within a magnet, providing a $0.2 \mathrm{~T}$ dipole field. The magnet is instrumented with the side range muon detector [51]. A schematic of ND280 is shown in Fig. 1.

The primary component of ND280 used in the analyses presented here is the central tracker region, comprising of three time projection chambers (TPCs) [52] and two fine grained detectors (FGD1 and FGD2) [53]. The FGDs are both instrumented with finely segmented scintillating bars which provide both charged particle tracking as well as a target mass for neutrino interactions and, whilst FGD1 is fully active, FGD2 also contains inactive water layers. In these analyses, only FGD1 is used as a hydrocarbon $\left(\mathrm{C}_{8} \mathrm{H}_{8}\right)$ target. Events leaving the FGDs can be tracked into the TPCs, which provide high-resolution tracking and thereby allow the curvature of charged particles to be used to make accurate measurements of their momenta (the TPCs provide an inverse momentum resolution of $10 \%$ at $1 \mathrm{GeV}$ ). This can then be combined with measurements of particle energy loss for charged particle identification (PID). If charged particles stop before leaving the FGD1, their momentum is determined by their length. In this case the PID is performed using both track length and the total energy-deposition. Muons and pions can also be identified by searching for delayed signal at the track end due to the Michel electron from the decay of muons (including muons from pion decay).

\section{MEASUREMENT STRATEGY}

\section{A. Observables}

This paper presents three different analyses which study the kinematics of the outgoing muon and protons in chargedcurrent events without pions in the final state $(\mathrm{CC} 0 \pi)$. Each of these analyses measures differential cross sections as a function of different observables and with a slightly different selection, optimized to the observables being measured.

The first "multidifferential" analysis measures the differential cross section as a function of the momentum and angle of the particles in the final state. This approach minimizes the dependency of the result on the input neutrino-nucleus scattering simulations, as will be described later, and provides the most complete information to characterize the final state. Such results can therefore be compared with present and future models of $\mathrm{CC} 0 \pi$ processes, even if their direct interpretation in terms of different nuclear effects is not straightforward. This multidimensional analysis simultaneously measures the cross section of events with and without detected protons in the final state, allowing a complete description of $\mathrm{CC} 0 \pi$ events and, due to improved constraints on systematic uncertainties, surpasses the accuracy of results previously reported by the T2K Collaboration in Ref. [44]. Since this analysis classifies events based on the number of reconstructed protons, it is also able to measure a cross section as a function of the multiplicity of protons above detection threshold. The other two analyses require the presence of at least one proton and, in the case where multiple protons are reconstructed, only the most energetic one is used to form the measured observables.

The second single transverse variables (STV) analysis measures the cross section of $\mathrm{CC} 0 \pi$ events with (at least) one proton in the final state as a function of the STV, which are defined in Ref. [46]. The MINERvA experiment are also measuring transverse kinematic imbalances with a $\sim 3 \mathrm{GeV}$ peak neutrino beam energy [54]. These variables are built specifically to characterize, and minimize the degeneracy between, the nuclear effects most pertinent to long-baseline oscillation experiments. In particular, the STV facilitate the possible identification of: Fermi motion of the initial state nucleon, final state reinteractions of the nucleons in the nucleus and multinucleon interactions (2p2h). As shown in Fig. 2, the STV are defined by projecting the lepton and proton momentum on the plane perpendicular to the neutrino direction. In the absence of any nuclear effects, the proton and muon momenta are equal and opposite in this plane and therefore the measured difference between their projections is a direct probe of nuclear effects in QE events:

$$
\delta \vec{p}_{\mathrm{T}}=\vec{p}_{\mathrm{T}}^{\mathrm{N}}-\Delta \vec{p}_{\mathrm{T}},
$$

where $\vec{p}_{\mathrm{T}}^{\mathrm{N}}$ is the initial state nucleon transverse momentum and $\Delta \vec{p}_{\mathrm{T}}$ is the modification due to final state effects. 


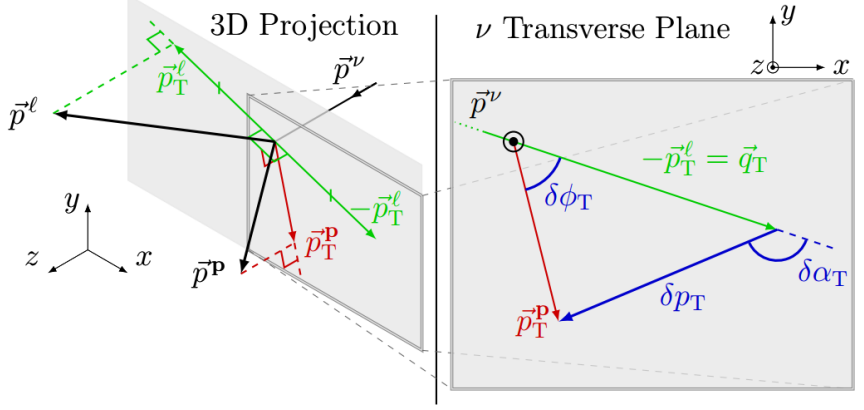

FIG. 2. Schematic view of the definition of the Single Transverse Variables: $\delta p_{\mathrm{T}}, \delta \alpha_{\mathrm{T}}$ and $\delta \phi_{\mathrm{T}}$. The left side shows an incoming neutrino interacting and producing a lepton $(\ell)$ and a proton $\mathrm{p}$, whose momenta are projected onto the plane transverse to the neutrino $(\nu)$. The right side then shows the momenta in this transverse plane and how the STV are formed from considering the imbalance within it. Taken from Ref. [55].

$\delta \vec{p}_{\mathrm{T}}$ can be fully characterized in terms of the vector magnitude $\left(\delta p_{\mathrm{T}}\right)$ and the two angles $\left(\delta \alpha_{\mathrm{T}}\right.$ and $\left.\delta \phi_{\mathrm{T}}\right)$ :

$$
\begin{gathered}
\delta p_{\mathrm{T}}=\left|\vec{p}_{T}^{l}+\vec{p}_{T}^{p}\right|, \\
\delta \alpha_{T}=\arccos \frac{-\vec{p}_{T}^{l} \cdot \delta \vec{p}_{T}}{p_{T}^{l} \delta p_{\mathrm{T}}}, \\
\delta \phi_{T}=\arccos \frac{-\vec{p}_{T}^{l} \cdot \vec{p}_{T}^{p}}{p_{T}^{l} p_{T}^{p}},
\end{gathered}
$$

where $p_{T}^{l}$ and $p_{T}^{p}$ are, respectively, the projections of the momentum of the outgoing lepton and proton on the transverse plane. Different nuclear effects alter the distributions of such STV in different and predictable ways. Measurements of the STV therefore have a unique sensitivity to identify nuclear effects, as will be exploited in Sec. V. This allows cross sections extracted using these observables to act as a powerful tool to tune and distinguish nuclear models. Furthermore, in case of disagreement the STV distributions provide useful hints on the possible causes of the discrepancies.

The third "inferred kinematics" analysis utilizes a similar kinematic imbalance to the STV analysis to probe nuclear effects in $\mathrm{CCO} \pi$ interactions by comparing the measured proton momentum and angle with the proton kinematics which can be inferred from the measured muon kinematics in the simplified QE hypothesis. Such inferred proton kinematics are estimated as follows:

$$
\begin{gathered}
E_{\nu}=\frac{m_{p}^{2}-m_{\mu}^{2}+2 E_{\mu}\left(m_{n}-E_{b}\right)-\left(m_{n}-E_{b}\right)^{2}}{2\left[\left(m_{n}-E_{b}\right)-E_{\mu}+p_{\mu} \cos \theta_{\mu}\right]}, \\
E_{p}^{\text {inferred }}=E_{\nu}-E_{\mu}+m_{p}, \\
\vec{p}_{p}^{\text {inferred }}=\left(-p_{\mu}^{x},-p_{\mu}^{y},-p_{\mu}^{z}+E_{\nu}\right),
\end{gathered}
$$

where the $\mathrm{z}$ axis corresponds to the neutrino direction, $n, p$, $\mu$ and $\nu$ denote the neutron, proton, muon and neutrino and
$E_{b}$ is the nuclear binding energy. The value of $E_{b}$ used in the definition of these variables is $25 \mathrm{MeV}$ for carbon, but this may be different from the event-by-event "physical" value of $E_{b}$. The cross section for events with a muon and (at least) one proton in the final state is then measured as a function of three observables:

$$
\begin{aligned}
\Delta p_{p} & =\left|\vec{p}_{p}^{\text {measured }}\right|-\left|\vec{p}_{p}^{\text {inferred }}\right|, \\
\Delta \theta_{p} & =\theta_{p}^{\text {measured }}-\theta_{p}^{\text {inferred }}, \\
|\Delta \mathbf{p}| & =\left|\vec{p}_{p}^{\text {measured }}-\vec{p}_{p}^{\text {inferred }}\right| .
\end{aligned}
$$

These observables are built such as to enhance nuclear effects which manifest themselves as deviations from zero imbalance. The STV depend only on transverse components of muon and proton momentum vectors with respect to the neutrino direction, while the variables of Eq. (8) depend also on the longitudinal components of both vectors. As such, there is no trivial relation between the two sets of variables such that each gives complimentary information about the nuclear effects involved in neutrino interactions. As can be seen in Eq. (7), the definition of the inferred proton kinematics relies on the same QE formula as is used in the estimation of neutrino energy in oscillations measurements at T2K. Therefore the observed deviations from the expected proton inferred kinematic imbalance provide hints of the biases that may be caused from the mismodeling of nuclear effects in neutrino oscillations measurements at T2K. The measurement of the differential cross section as a function of these proton inferred kinematic variables is performed separately in bins of muon kinematics. This can highlight the possible mismodeling of nuclear effects in different regions of the muon kinematic phase space and is also essential in order to mitigate the model dependence in the efficiency corrections (this will be further discussed in Sec. III B). Once de-convoluted from detector effects, this analysis measures how the true particle kinematics deviate from their inferred values under a $\mathrm{QE}$ approximation.

\section{B. Minimization of input-model dependence}

In all three analyses, extensive precautions are taken to ensure that the results are minimally dependent on the signal model used in the reference $\mathrm{T} 2 \mathrm{~K}$ simulation (this model is detailed in Sec. IV). This is particularly important for these analyses since the predictive power of available interaction models for the outgoing proton kinematics, and the relative kinematics between muon and protons, is poor. One crucial way to minimize such model-dependence is to ensure that the analyses' signal definition is only reliant on observables which are experimentally accessible at ND280. As such, the signal is defined as all events with no pions in the final state $(\mathrm{CC} 0 \pi)$ without correcting for FSI pion absorption. Moreover, for the analyses which integrate over large regions of kinematic phase space or do not estimate the efficiency as a function of all 
TABLE I. Signal phase-space restrictions for the three analyses. The cuts apply to the proton with the highest momentum.

\begin{tabular}{lcccc}
\hline \hline Analysis & $p_{p}$ & $\cos \theta_{p}$ & $p_{\mu}$ & $\cos \theta_{\mu}$ \\
\hline Multidimensional 0p & $<500 \mathrm{MeV}$ (or no proton) & $\ldots$ & $\ldots$ & $\ldots$ \\
Multidimensional 1p & $>500 \mathrm{MeV}$ & $\ldots$ & $\ldots$ & $\ldots$ \\
STV & $0.45-1 \mathrm{GeV}$ & $>0.4$ & $>250 \mathrm{MeV}$ & $>-0.6$ \\
Inferred kinematics & $>450 \mathrm{MeV}$ & $>0.4$ & $\ldots$ & $\ldots$ \\
\hline \hline
\end{tabular}

relevant kinematic variables, it is also absolutely necessary to apply phase-space restrictions in the signal definition in order to avoid model dependence in the efficiency correction. The phase-space restrictions used in the analyses presented here are shown in Table I. Since the efficiency of detecting muons and protons in ND280 is not flat as function of the particles angle and momentum, the efficiency correction should be made as a function of the momentum and angle of both the outgoing particles. The relative angle between the outgoing particles is also important but, due to the magnetic field and the very good spatial resolution of the TPCs, this has only a second-order effect on the efficiency. The multidifferential analysis performs a complete multidimensional efficiency correction and therefore only a loose phase-space restriction on the proton momentum is applied. The STV analysis may, in principle, be the most affected by this issue since each bin of the STV integrates over all possible muon and proton kinematics. As a consequence, the STV measurements use the most stringent restrictions in the signal phase space, selecting only regions of flat and/or well understood efficiency. Finally, the inferred kinematics analysis performs a measurement binned in muon momentum and angle and thus it requires only restrictions on the proton phase space. It should be noted that the restrictions listed in Table I are applied in the signal identification at generator level, therefore the multiplicity of the protons is defined counting only protons above the thresholds in the table. The final measurements do not correct for protons which cannot be detected efficiently and therefore the same restrictions have to be applied to any model in order to compare with the results presented in this paper.

To further alleviate model-dependence, the measured differential cross sections are flux-integrated, normalizing all the bins of the measured variables to the same flux:

$$
\frac{d \sigma}{d x_{i}}=\frac{N_{i}^{C C 0} \pi}{\epsilon_{i} \Phi N_{\text {nucleons }}^{\mathrm{FV}} \Delta x_{i}},
$$

where $N_{i}^{C C 0 \pi}$ is the measured number of signal events in the $i$-th bin, $\epsilon_{i}$ is the efficiency in that bin, $\Phi$ is the overall flux integral, $N_{\text {nucleons }}^{\mathrm{FV}}$ is the number of nucleons in the fiducial volume and $x$ is the measured variable.

The analyses can be further affected by model-dependent assumptions in the process of correcting for detector effects. The multidimensional and the STV analyses use a binned likelihood fit, similar to that used for Analysis I in Ref. [44]. The results of this method, when unregularized, are completely independent on the nominal model used to create the reference templates for the signal. The STV analysis also provides results after applying a regularization method which has been tuned and thoroughly tested in order to minimize the dependence on the signal model. The third analysis exploits the D'Agostini unfolding procedure [56,57], also described for Analysis II in Ref. [44].

To additionally reduce model-dependence, and to minimize systematic uncertainties related to background modeling, each analysis employs dedicated control regions to achieve a data-driven background estimation and subtraction. Since the control regions chosen and the background subtraction method differs slightly between analyses, these will be discussed in the details of the strategy for each of the analyses which will be reported in Sec. IV.

Despite the many aforementioned precautions, it is still possible that residual model dependence can bias analysis results. To ensure this does not happen, a comprehensive set of studies with mock data sets has been performed. A first set of mock data sets is created by modifying systematic parameters of particular interest within the reference model (for example $2 \mathrm{p} 2 \mathrm{~h}$ normalization or $M_{A}^{\mathrm{QE}}$ ). The cross-section extraction methods must be able to recover the truth when each mock data set is treated exactly as real data. However, this only tests that the methods can extract the truth from mock data which are systematic variations of the input model, and so is more of a closure test than a true evaluation of possible bias. For a more rigorous test, alternative Monte Carlo event generators, which employ some entirely different signal and background models, are used to produce mock data. Moreover, some of these mock data is specialized to specifically modify the models of the nuclear effects that the analyses wish to characterize, namely modifying $2 \mathrm{p} 2 \mathrm{~h}$ shape, Fermi motion and FSI models. Using such mock data as an input, it has been verified that, even in the case of extreme deviations from the input signal model, the cross-section extraction machinery for each analysis can recover the truth such that it is always well within the uncertainties on the extracted result and also produces a small $\chi^{2}$ when the full resultant covariances are considered. Some examples of such studies can be found in [58].

Finally, it should be noted that the three analyses exploit the same data and rely on similar selections. The systematic uncertainties are also evaluated in similar ways, for instance relying on the same data in control regions. As a consequence, it is a very good approximation to assume all the uncertainties to be fully correlated between the different 
analyses thus the results of the analysis should not be used together in a joint fit. A full discussion on the interpretation of the results will be reported in Sec. V.

\section{ANALYSES DESCRIPTION AND RESULTS}

\section{A. Simulation}

The analysis of the neutrino data relies on simulation in order to correct the measured quantities for flux normalization, for detector effects and to estimate the systematic uncertainties.

The T2K flux simulation is based on the modeling of interactions of protons with a graphite target using the FLUKA 2011 package [59,60]. The modeling of hadron reinteractions and decays outside the target is performed using GEANT3 [61] and GCALOR [62] software packages. Multiplicities and differential cross sections of produced pions and kaons are tuned based on the NA61/SHINE data [63-65] and on other experiments [66-68], allowing the reduction of the overall flux normalization uncertainty to $8.5 \%$.

The neutrino interaction cross section with nuclei in the detector and the kinematics of the outgoing particles are simulated by the T2K neutrino event generator NEUT 5.3.2 $[69,70]$. The final state particles are then propagated through the detector material using GEANT4 [71]. Various additional neutrino event generators are used in the analyses presented in this paper in order to both test the robustness of the results (as discussed in Sec. III B) and to compare the final measurements to different models. To this end, NEUT 5.3.2.2, NEUT 5.4.0, GENIE 2.12.4 [72], GENIE 2.8.0, NuWro 11q [73], and GIBUU 2016 [74] are used.

NEUT version 5.3.2 utilizes the Llewellyn-Smith formalism [75] to describe the CCQE neutrino-nucleon cross section and the spectral function (SF) from Ref. [76] is used as a nuclear model. The axial mass used for quasielastic processes $\left(M_{A}^{\mathrm{QE}}\right)$ is set to $1.21 \mathrm{GeV}$, based on the SuperKamiokande measurement of atmospheric neutrinos and the $\mathrm{K} 2 \mathrm{~K}$ measurement on the accelerator neutrino beam [18], while the resonant pion production process is described by the Rein Sehgal model [77] with the axial mass $M_{A}^{\mathrm{RES}}$ set to $1.21 \mathrm{GeV}$. The simulation of multinucleon interactions, when the neutrino interacts with a correlated pair of nucleons, also called $2 \mathrm{p} 2 \mathrm{~h}$ interactions, is based on the model from Nieves et al. in Ref. [78].

The deep inelastic scattering (DIS), relevant at neutrino energy above $1 \mathrm{GeV}$, is modeled using the parton distribution function GRV98 [79] with corrections by Bodek and Yang [80]. The FSI, describing the transport of the hadrons produced in the elementary neutrino interaction through the nucleus, are simulated using a semiclassical intranuclear cascade model.

A different version of NEUT (5.3.2.2) is used in the comparison of the final results with the models, which differs from the version used for the main analysis of the data by its different value of $M_{A}^{\mathrm{QE}}=1.03 \mathrm{GeV}$ and its more realistic, reduced strength of proton FSI. NEUT additionally 5.3.2.2 facilitates the alteration of nucleon FSI strength by varying the mean free path between FSI during the intranuclear cascade. The final results are also compared to a third NEUT version (NEUT 5.4.0) where a fully consistent local Fermi gas (LFG) $1 \mathrm{p} 1 \mathrm{~h}$ and $2 \mathrm{p} 2 \mathrm{~h}$ model based on the work of Nieves et al. in Ref. [78] has been implemented.

GENIE, an alternative neutrino generator exploited in these analyses, uses different values of the axial masses $\left(M_{A}^{\mathrm{QE}}=0.99 \mathrm{GeV}\right.$ and $\left.M_{A}^{\mathrm{RES}}=1.12 \mathrm{GeV}\right)$ and relies on a different nuclear model for $\mathrm{CCQE}$ events: a relativistic Fermi gas (RFG) with Bodek and Ritchie modifications [81]. A parametrized model of FSI is used (known as GENIE's "hA" model). Both GENIE 2.8.0 and 2.12.4 are used within the analyses, the latter facilitates the optional inclusion of $2 \mathrm{p} 2 \mathrm{~h}$ interactions using the so-called "empirical" MEC model alongside other improvements to the FSI model.

The NuWro 11q version is also used in these analyses. It simulates the CCQE process with the Llewellyn-Smith model, assuming an axial mass $M_{A}^{\mathrm{QE}}=1.0 \mathrm{GeV}$, and the $2 \mathrm{p} 2 \mathrm{~h}$ process by the model in Ref. [78], similarly to NEUT. Different nuclear models are considered in the comparison to the data: SF, RFG and LFG. For LFG and RFG the effect of random phase approximation (RPA) corrections, as computed in Ref. [32], is tested. RPA is not applied to SF since the model already partially contains the short- and long-range correlations between the nucleons in the nucleus. Similarly the $2 \mathrm{p} 2 \mathrm{~h}$ contribution should be different in SF with respect to what has been calculated in Ref. [32] for LFG. However, since a dedicated computation of the 2-body current for the SF is not yet available in simulations, the same $2 \mathrm{p} 2 \mathrm{~h}$ contribution as in LFG is added on top of the SF in both the NEUT and NuWro simulations. For pion production a single $\Delta$ model by AdlerRarita-Schwinger is used for the hadronic mass $W<$ $1.6 \mathrm{GeV}$ with $M_{A}^{\mathrm{RES}}=0.94 \mathrm{GeV}$. A smooth transition to deep inelastic processes is made for $W$ between 1.3 and $1.6 \mathrm{GeV}$. The total cross section for DIS is based on the Bodek and Yang approach, similarly to other generators. Like NEUT the FSI are simulated with a semiclassical cascade model.

The measurements presented in this paper are also compared to GiBUU 2016 where the Giessen-BoltzmannUehling-Uhlenbeck implementation of quantum-kinetic transport theory [82] is used. The nucleons are inserted in a coordinate- and momentum-dependent potential using the LFG momentum distribution. The CCQE process is modeled as in Ref. [83] with $M_{A}^{\mathrm{QE}}=1.03 \mathrm{GeV}$. The $2 \mathrm{p} 2 \mathrm{~h}$ contribution is simulated by considering only the transverse contributions and translating to neutrino scattering the response measured in electron scattering [74]. In these comparisons the default GiBUU 2016 initial state isospin for $2 \mathrm{p} 2 \mathrm{~h}$ interactions is used $(\mathcal{T}=1)$. The model used for single pion production [84] mostly differs from the other generators for the inclusion of medium effects on the $\Delta$ resonance. The DIS is simulated with PYTHIA v6.4.

The comparison of the measurements presented in this paper to the various mentioned models is performed in the framework of NUISANCE [85]. 


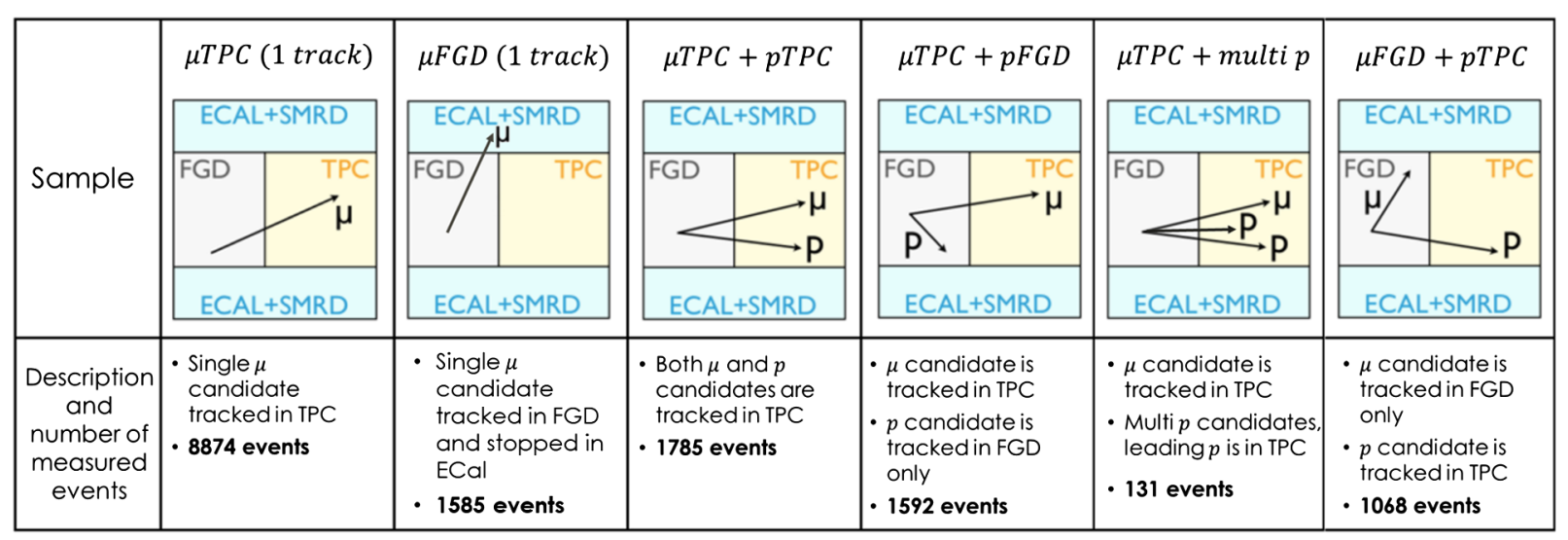

FIG. 3. A diagram summarizing the different signal samples used. The number of events selected in data for each sample is indicated.

\section{B. Event selection}

The three analyses presented herein share a common basic event selection, which aims to identify muon neutrino interactions with a hydrocarbon target producing one muon, no pions and any number of protons in the final state. Events are pre-selected by identifying a vertex in the most upstream fine-grained detector (FGD1) associated with either the highest momentum negative track in the central TPC or, if there is no negative track, the highest momentum positive track. If there is no such TPC track the event is rejected. This pre-selection is split depending on the charge of this primary track, as shown in Fig. 3.

If the primary track is negative then the track is required to be muonlike using the TPC PID. Extra tracks sharing a common vertex with the primary track must either have good quality measurement in the TPC, or be contained in FGD1 such that their kinematics can be reliably determined and they must be identified as protonlike by the TPC or FGD PID respectively. If there is more than one extra track sharing such a common vertex then it is required that at least one of these tracks enter the TPC but each must be identified as protonlike. Following this selection, any events with other tracks that are not muon- or protonlike are rejected. To reject events with low momentum charged or neutral pions, it is required that no Michel electrons (electrons from the decay of the muon that itself is from the pion decay) are tagged within the FGD and that there is no activity in the tracker ECal consistent with a photon. The selected events are then split into samples based on whether there was zero, one or more than one protonlike track and, if so, whether it left a track in the TPC.

If the primary track is positive (and there are therefore no identified negative TPC tracks) then the selection requires the identification of a single extra FGD track sharing a common vertex position with the primary track. This track must then either stop in FGD1 or in the surrounding ECal and be identified as muonlike by the FGD or ECal PID respectively. In the latter case, time of flight information between FGD1 and the ECal is used to ensure that energy depositions seen in the ECal are related to the same track that traversed the FGD.

Finally a last sample is selected with a single track traveling through the FGD before stopping in the ECal. This sample uses the measured time of flight between the track ends to verify propagation direction, and the ECal hit topologies to verify whether the track is muonlike. This is a small sample but all concentrated at high angle, therefore it is included only in the multidifferential analysis which measures the cross section with finer binning in muon angle.

Figure 3 summarizes the topology and the number of selected events within the six signal samples discussed while the number of selected events in each sample, broken down by true interaction topology, is shown in Fig. 4. Other samples are possible but typically with very poor efficiency, resolution and larger detector systematic uncertainties, for example events with a negative primary track and multiple FGD1 contained protons. Since such alternative samples are found to make up a very small number of selected events, less than 30 events in the available data, they are excluded.

As discussed in Sec. III B, it is important not to attempt to correct for low efficiency in regions of kinematic phasespace that the detector is not sensitive to. This is particularly important when measuring a differential cross section in observables that do not well characterize a detector's acceptance such as the single-transverse and proton inferred kinematic observables. To avoid input-model bias from integrating over regions of changing efficiency, it is necessary to set appropriate limitations on the kinematic phase space of the final state particles. In the analyses presented here, both muons and protons are identified and therefore ND280's acceptance is reasonably well characterized by the muon and proton momentum and angle. ${ }^{1}$ Ideally, the

\footnotetext{
${ }^{1}$ It should be noted that ND280's acceptance has also a small dependence on other factors, most markedly the vertex position and the angle between the outgoing muon and proton. However, the distribution of the former is not dependent on the interaction model while, as discussed in Sec. III B, the impact of the latter is fairly small.
} 


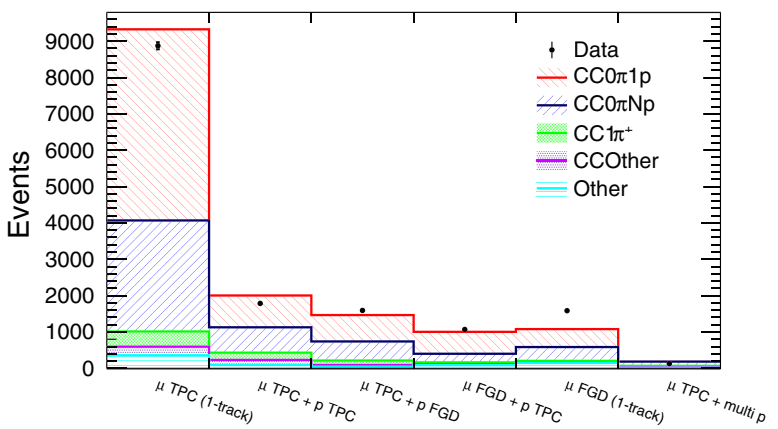

FIG. 4. The number of selected events in each sample of the event selection within data and the NEUT 5.3.2 simulation. The simulated events are broken down by true interaction topology as predicted by the generator.

selected phase-space restrictions should leave a flat efficiency within the four dimension regions of muon- and proton-kinematics which will be integrated over in the final measurement. This ensures that the efficiency corrections are independent of the distribution of kinematics which are not measured. To determine the phase-space restrictions
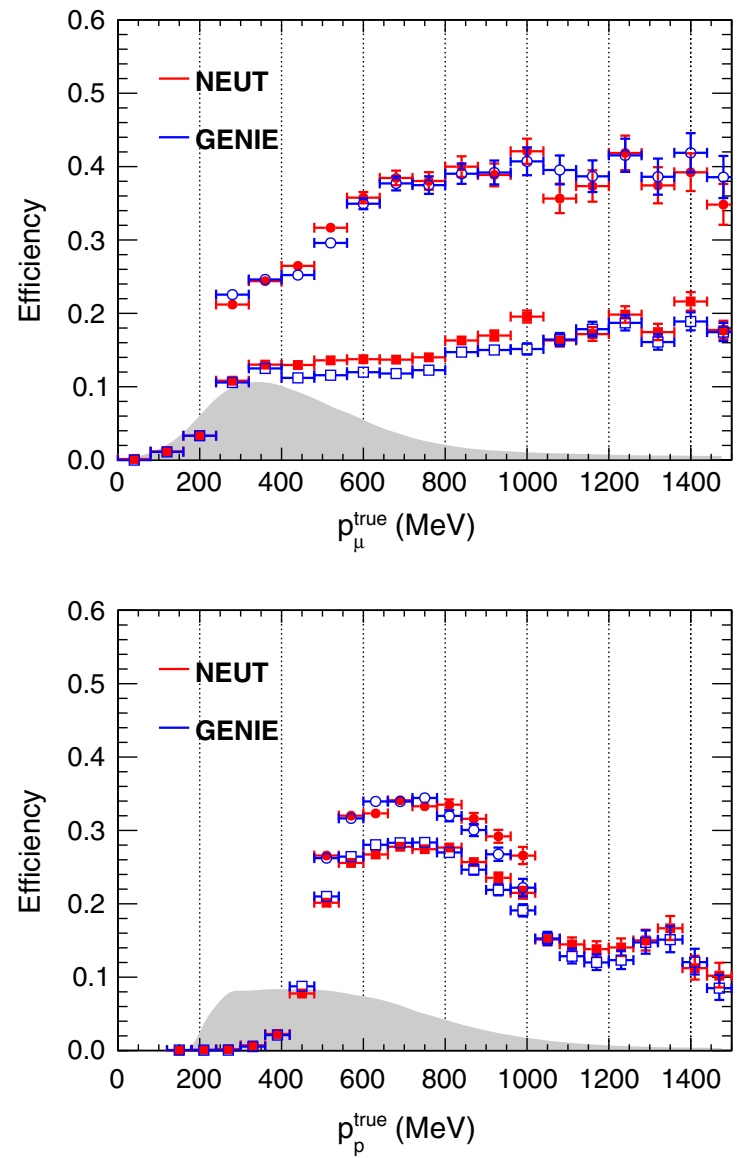

introduced in Table I, the efficiency and selected event distributions were studied in various projections of the underlying four-dimensional kinematics in order to find a suitable balance between efficiency flatness and the number of $\mathrm{CC} 0 \pi+\mathrm{Np}$ events that fall out of the restricted phase space (which are then considered as background). The resultant impact of the phase-space restrictions is shown for both the ND280 NEUT 5.3.2 and GENIE 2.8.0 simulations in Fig. 5, which shows the efficiency after all the selection steps projected into the relevant kinematic variables, before and after phase-space restrictions are applied. In general it can be seen that the chosen phase-space restrictions ensure a more flat efficiency within the regions of kinematic phase space that contribute most to the $\mathrm{CC} 0 \pi$ cross section, particularly in the poorly understood outgoing proton kinematics.

Following the event selection and the application of the phase-space restrictions in both true and reconstructed kinematics, the efficiency and purity of signal events for each analysis is shown in Table II. The reconstructed muon and proton kinematics from the combined samples, broken
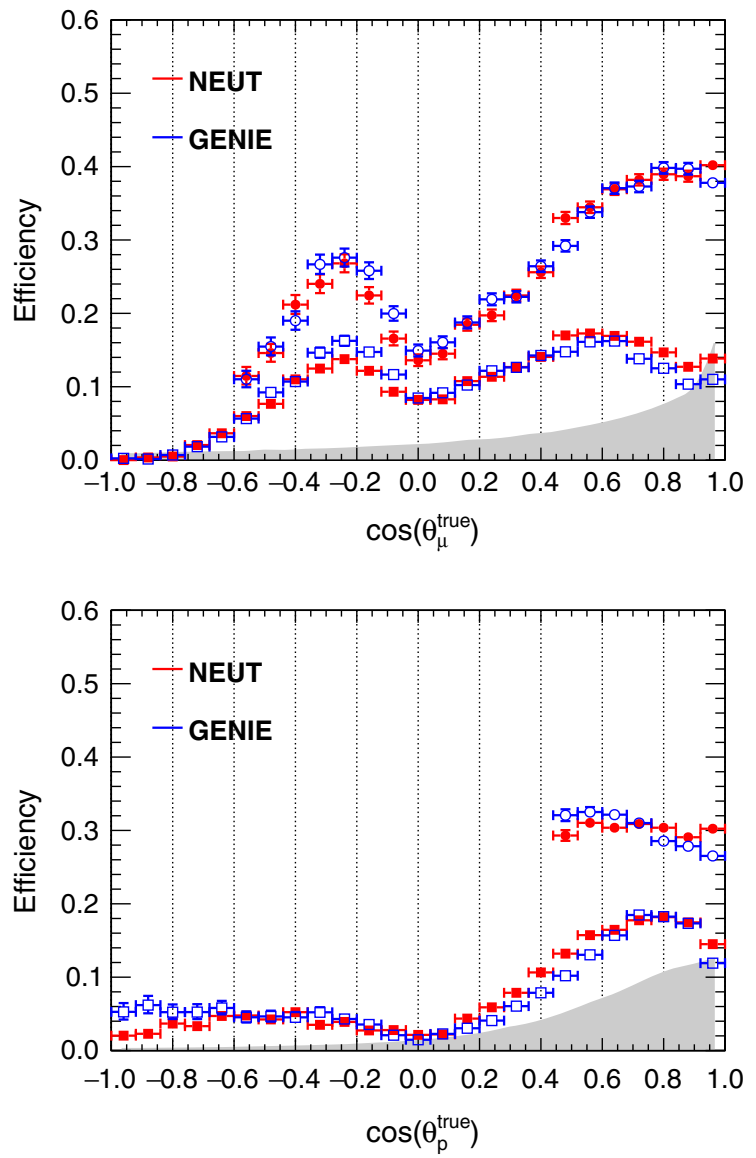

FIG. 5. Efficiencies, after all the selection steps, as function of true muon (upper plots) and proton (lower plots) kinematics as predicted by NEUT 5.3.2 and GENIE 2.8.0. The square points show the efficiency prediction before any phase-space constraints whilst the circular points have had the proton and muon kinematic constraints for the STV analysis in Table I applied. The grey filled distribution shows the shape of the $\mathrm{CC} 0 \pi$ cross section predicted by NEUT 5.3.2.2. 
TABLE II. The purity, the efficiency (both from NEUT 5.3.2 and GENIE 2.8.0) and the number of selected events in data for each analysis in the restricted phase and before phase-space restriction (unconstrained).

\begin{tabular}{lccc}
\hline \hline Analysis & Purity & Efficiency & Events \\
\hline Multidifferential & $78.3 \%$ & $20.5 \%$ & 3674 \\
Inferred kinematics & $79.4 \%$ & $21.0 \%$ & 3691 \\
STV & $80.7 \%$ & $24.1 \%$ & 3073 \\
Unconstrained & $81.2 \%$ & $12.3 \%$ & 4576 \\
\hline \hline
\end{tabular}

down by topology, are shown in Fig. 6. The events are separated depending if, based in their true kinematics properties, they fall in or out of the phase-space restrictions (IPS/OOPS) listed in Table I. The distribution of the singletransverse and inferred kinematic observables are also shown in Fig. 7.

Although the selection presented in this chapter identifies a high purity sample of $\mathrm{CC} 0 \pi+\mathrm{Np}$ events, there are still non-negligible backgrounds. The majority of these come from $\mathrm{CC} 1 \pi^{+}$events, where the pion (and associated Michel electron) are missed, but there is also notable contribution from other (multipion) CC events. These backgrounds are constrained through dedicated control samples which allow an improved background estimation and thereby smaller background modeling uncertainties.
In the multidifferential and STV analyses two control samples are employed for the background constraint. Both require the identification of a negatively charged muonlike track and a positively charged pionlike track in the TPC and are split depending on whether there are any extra tracks sharing a common vertex with the identified muon and pion candidates. The vertex must be contained in the FGD1. These control regions will be referred to as $\mathrm{CC} 1 \pi^{+}$and CCOther respectively. An illustration of the topologies these aim to identify is shown in Fig. 8 while the distribution of the data and simulated events within each control sample are shown in Figs. 9 and 10.

These figures highlight an initial large discrepancy between the NEUT prediction and the data, particularly in the $\mathrm{CC} 1 \pi^{+}$sample. This is understood to primarily come from an overestimation of the contribution from neutrino induced coherent pion production, as demonstrated in Ref. [86]. However, the likelihood fit used in the multidifferential and the STV analyses allows to adapt the NEUT model to the data within the control regions. The postfit NEUT prediction from the likelihood fit performed in the $\delta p_{\mathrm{T}}$ measurement is shown in the figures to be in much better agreement with the data (similar results are also obtained in the other STV and multidifferential analyses).
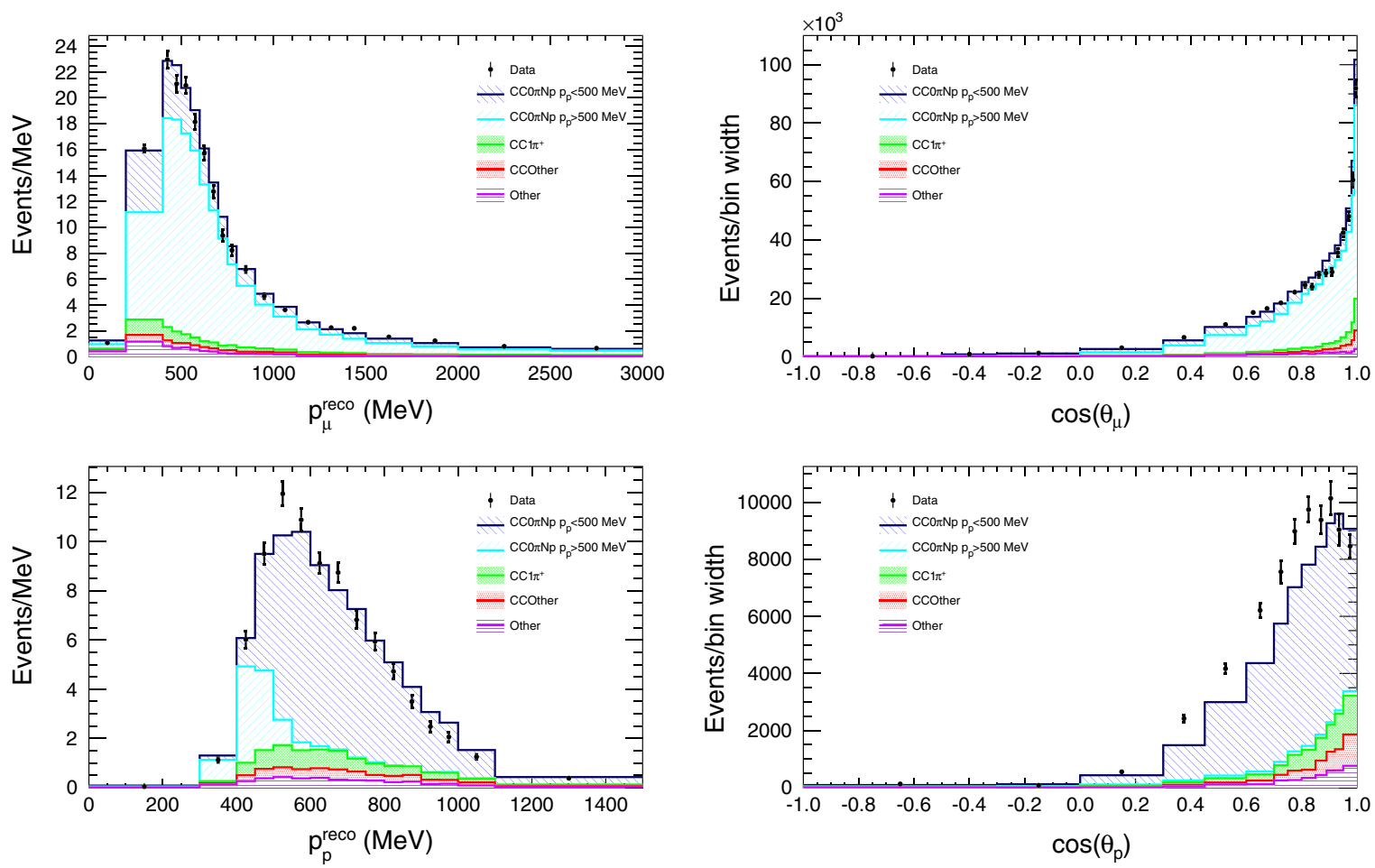

FIG. 6. The distribution of reconstructed observables used within the multidifferential analyses following the event selection for both NEUT 5.3.2 and data. The muon kinematic plots show events from all samples while the proton kinematics are limited to showing events from the samples which identify a proton. The plots are broken down by interaction topology and the $\mathrm{CC} 0 \pi$ contribution is further split depending on whether the interaction falls within the multidifferential analysis phase-space constraints from Table I. 

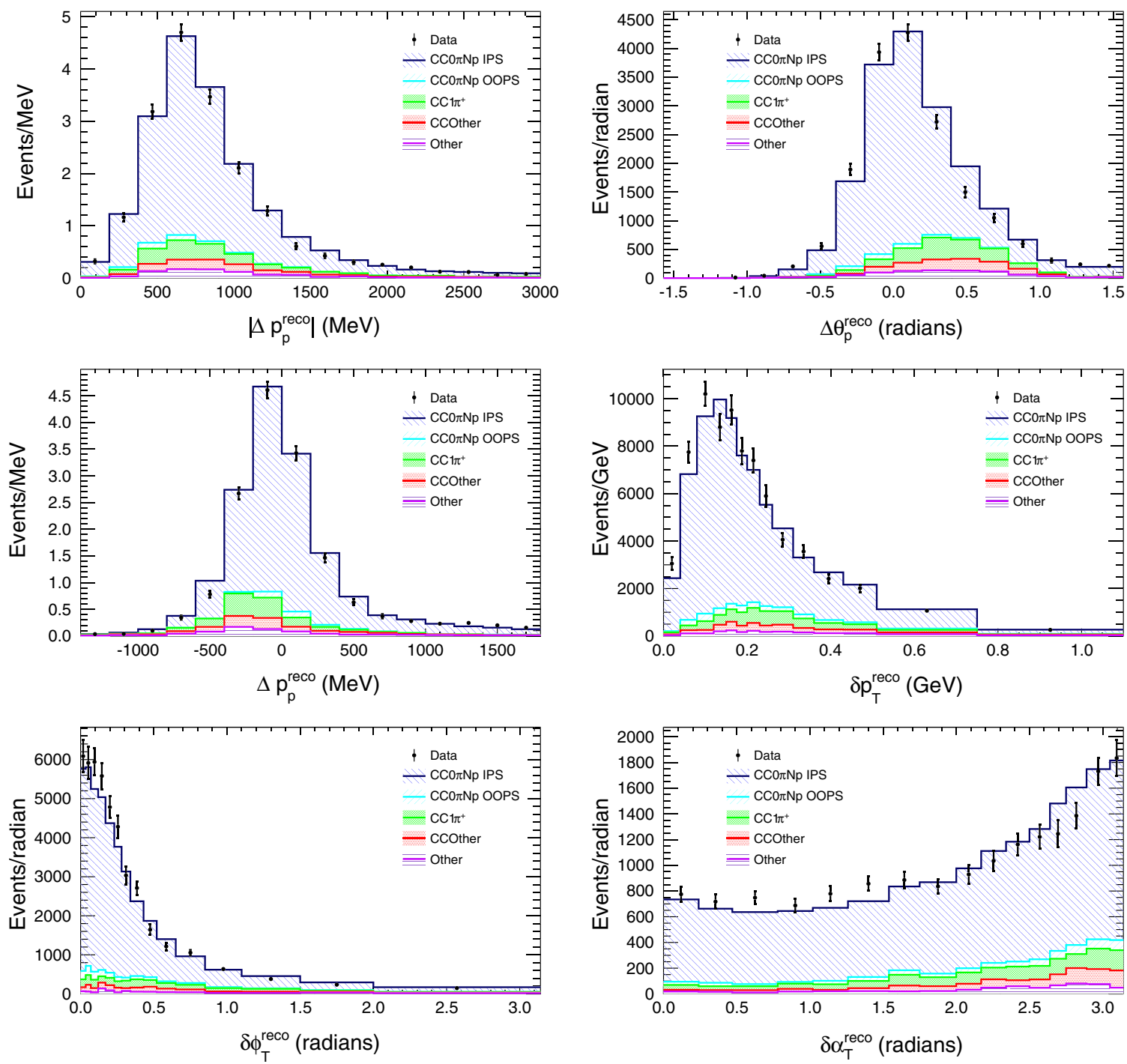

FIG. 7. The distribution of reconstructed observables used within the inferred kinematics and STV analyses following the event selection and phase-space restrictions on the reconstructed kinematics for both NEUT 5.3 .2 and data. The plots are broken down by interaction topology and the $\mathrm{CC} 0 \pi$ contribution is further split depending on whether the interaction falls in or out of the phase-space constraints (IPS/OOPS) from Table I (the phase-space definitions for the STV or inferred kinematics plots follow the restrictions pertinent to their respective analyses).

In the inferred kinematics analysis, a control sample is built by inverting the cut on Michel electrons in the signal samples. The resultant kinematic distributions of the selected data and MC events are shown in Fig. 11. This control sample is then unfolded simultaneously with the signal regions to constrain the background.

\section{Sources of systematic uncertainties}

The measurements presented in this paper account for the following systematic uncertainties:

(i) neutrino flux uncertainty. The flux simulation is tuned using external hadron-production measurements and INGRID monitoring, as discussed in Sec. IVA. The residual flux uncertainties affect the cross-section measurements presented in this paper, mainly through an overall normalization uncertainty of approximately $8.5 \%$.

(ii) detector effects (efficiency and resolution) which are not perfectly reproduced in the simulation. To evaluate such uncertainties the simulation is compared to the data in dedicated and independent control samples, any observed bias is corrected and the statistical uncertainties in such data and simulated samples are used as residual uncertainties.

(iii) modeling of the signal and background interactions, including nuclear effects. As previously discussed in Sec. III B, a possible model-dependent bias may be introduced in the multidifferential and STV analyses through efficiency corrections, while 


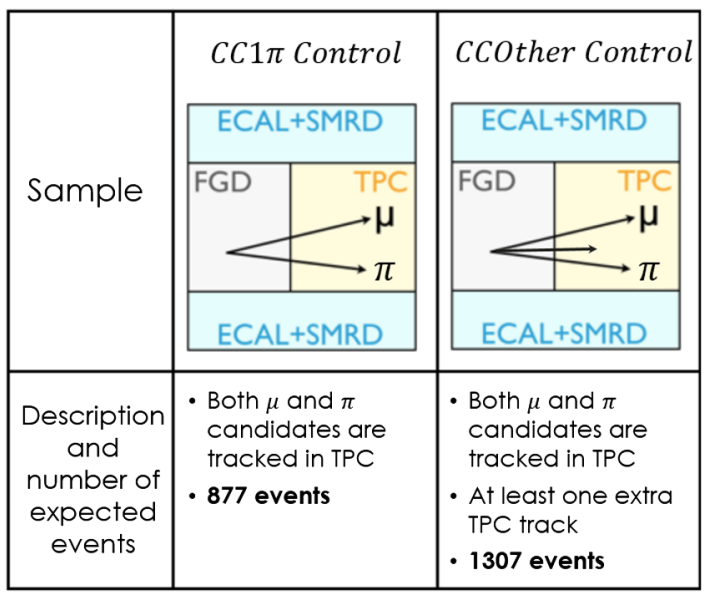

FIG. 8. A diagram summarizing the different control samples used. The number of events selected in data for each sample is indicated.

the measurement of proton inferred kinematics is also affected through the unfolding procedure and the simulation-based background corrections. Such effects are covered by dedicated systematic uncertainties which are quantified by evaluating the variation of the measured cross section using modified simulation models; the theory parameters describing the signal and the background, including proton and pion FSI, are varied inside their prior uncertainty, based on theory expectations and comparisons to external data.

Such uncertainties are implemented in the cross-section extraction in different ways in each of the analyses presented in this paper, as will be described in the following sections. In general the systematic parameters considered and their variation is similar to that used for the near detector fit of $\mathrm{T} 2 \mathrm{~K}$ oscillation analyses described in Ref. [9]: the most notable differences being the inclusion of proton FSI uncertainties and the usage of Gaussian priors for the parameters describing CCQE uncertainties.

\section{Method of cross-section evaluation}

Each of the analyses take different approaches when extracting a cross section from the selected events detailed in Sec. IV B. All of these methods involve an effective background subtraction; an efficiency correction; and the deconvolution of detector effects either by a binnedlikelihood fit for the multidifferential and STV analyses, or an iterative unfolding procedure for the analysis of the inferred kinematics.

\section{Binned likelihood fitting}

In order to produce a data spectrum that is de-convoluted from detector smearing, the input simulation is varied via a set of parameters, such that a best-fit set can be extracted once the simulation best describes the observed
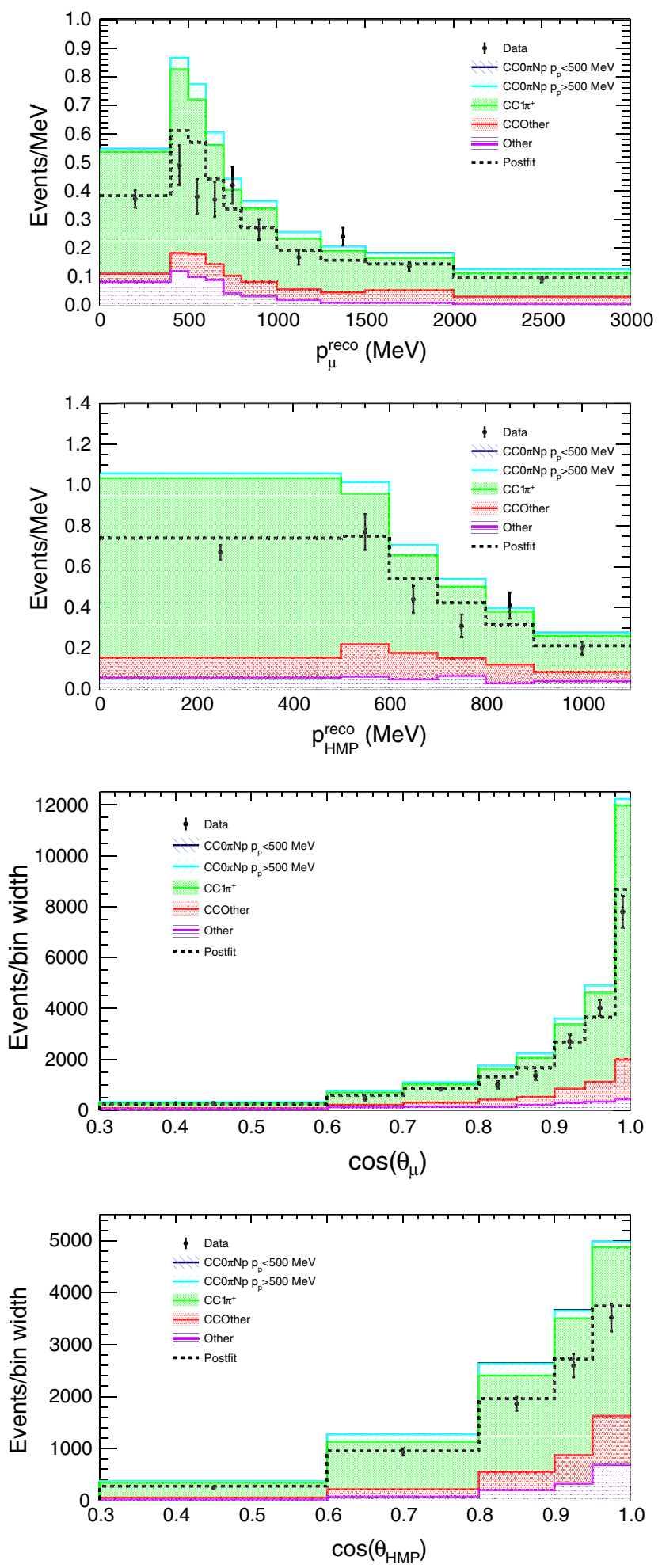

FIG. 9. The reconstructed kinematics of the muon and of the highest momentum positive (HMP) hadron for events selected within the $\mathrm{CC} 1 \pi^{+}$control region from both data and NEUT 5.3.2. The plots are broken down by interaction topology and the $\mathrm{CC} 0 \pi$ contribution is further split depending on whether the interaction falls within the multidifferential analysis phase-space constraints from Table I. The postfit NEUT prediction from the likelihood fit to extract a cross section as a function of $\delta p_{\mathrm{T}}$ is also shown. 

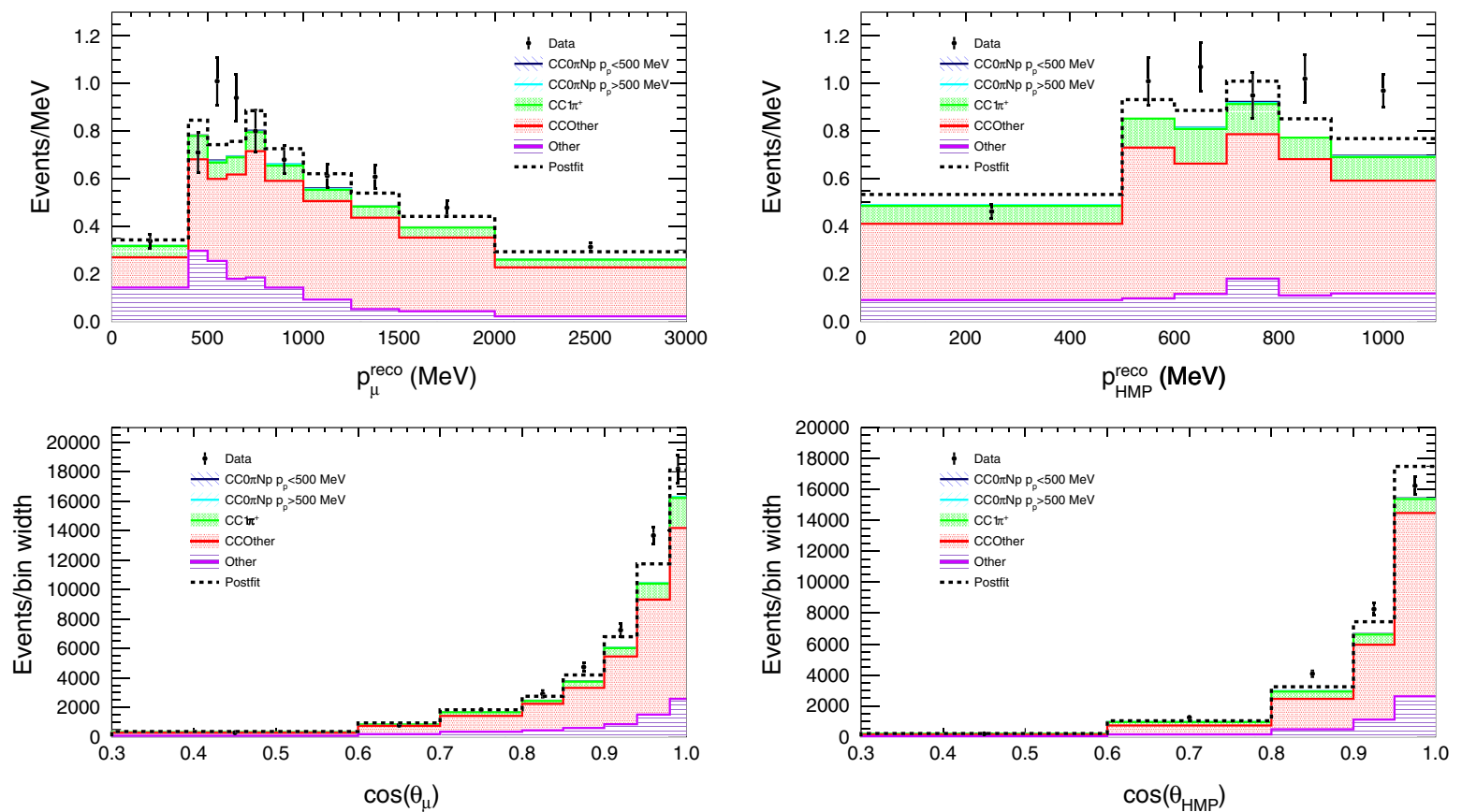

FIG. 10. The reconstructed kinematics of the muon and of the highest momentum positive (HMP) hadron for events selected within the CCOther control region from both data and NEUT 5.3.2. The plots are broken down by interaction topology and the CC0 $\pi$ contribution is further split depending on whether the interaction falls within the multidifferential analysis phase space constraints from Table I. The postfit NEUT prediction from the likelihood fit to extract a cross section as a function of $\delta p_{\mathrm{T}}$ is also shown.

data. The signal is parametrized using "template signal weights" $\left(c_{i}\right)$ which alter the number of selected signal events in bins $(i)$ of some truth-level observable(s) with no prior constraint. Parameters describing plausible systematic variations of the flux, detector response and background processes can also be fit simultaneously to the signal parameters. The effect of these parameter variations is then propagated through to the number of selected events in reconstructed bins of the same observable (using the expected smearing due to detector resolution and efficiency), such that the updated simulation prediction can be compared to the data. The best-fit set of parameters are chosen by minimizing the following negative log-likelihood:

$$
-2 \log (L)=-2 \log \left(L_{\text {stat }}\right)-2 \log \left(L_{\text {syst }}\right) .
$$

Where:

$$
-2 \log (L)_{\text {stat }}=\sum_{j}^{\text {reco bins }} 2\left(N_{j}^{\text {sim }}-N_{j}^{\text {obs }}+N_{j}^{\text {obs }} \log \frac{N_{j}^{\text {obs }}}{N_{j}^{\text {sim }}}\right),
$$

and

$$
-2 \log (L)_{\text {syst }}=\left(\vec{a}^{\text {syst }}-\vec{a}_{\text {prior }}^{\text {syst }}\right)\left(V_{\text {prior }}^{\text {syst }}\right)^{-1}\left(\vec{a}^{\text {syst }}-\vec{a}_{\text {prior }}^{\text {syst }}\right) .
$$

The term in Eq. (11) is the Poisson likelihood, where $N_{j}^{\text {sim }}$ and $N_{j}^{\text {obs }}$ are the number of simulated and observed events in each reconstructed bin, $j$. The term in Eq. (12) characterizes the prior knowledge of the values of the systematic parameters $\left(\vec{a}^{\text {syst }}\right)$ and their correlations, as a multivariate Gaussian likelihood where $\vec{a}_{\text {prior }}^{\text {syst }}$ are the prior values of these parameters and $V_{\text {prior }}^{\text {syst }}$ is a covariance matrix describing the correlations between them.

As described above, $N_{j}^{\text {sim }}$ is described by alterations to the nominal input simulation based on the template signal weights and the systematic fit parameters,

$$
N_{j}^{\text {sim }}=\sum_{i}^{\text {true bins }}\left(c_{i} w_{i}^{\text {sig }} N_{i}^{\text {sim sig }}+w_{i}^{\text {bkg }} N_{i}^{\text {sim bkg }}\right) U_{i j},
$$

where $N_{i}^{\text {simsig }}$ and $N_{i}^{\text {simbkg }}$ are the number of signal and background events in true bin $i$ of the input simulation; $c_{i}$ are the signal template weights; $w_{i}^{\text {signal }}$ and $w_{i}^{\text {bkg }}$ describe the alterations to the input simulation from the aforementioned systematic parameters; and $U_{i j}$ is the smearing matrix describing the probability of finding an event in true bin $i$ in reconstructed bin $j$. This smearing matrix is also subject to change with the alteration of systematic parameters.

The result of the fit is the $N_{i}^{C C O \pi}$ term from Eq. (9): the number of selected signal events deconvoluted from detector smearing in each analysis bin. As shown in Eq. (9), this must then account for the integrated T2K flux, the number 

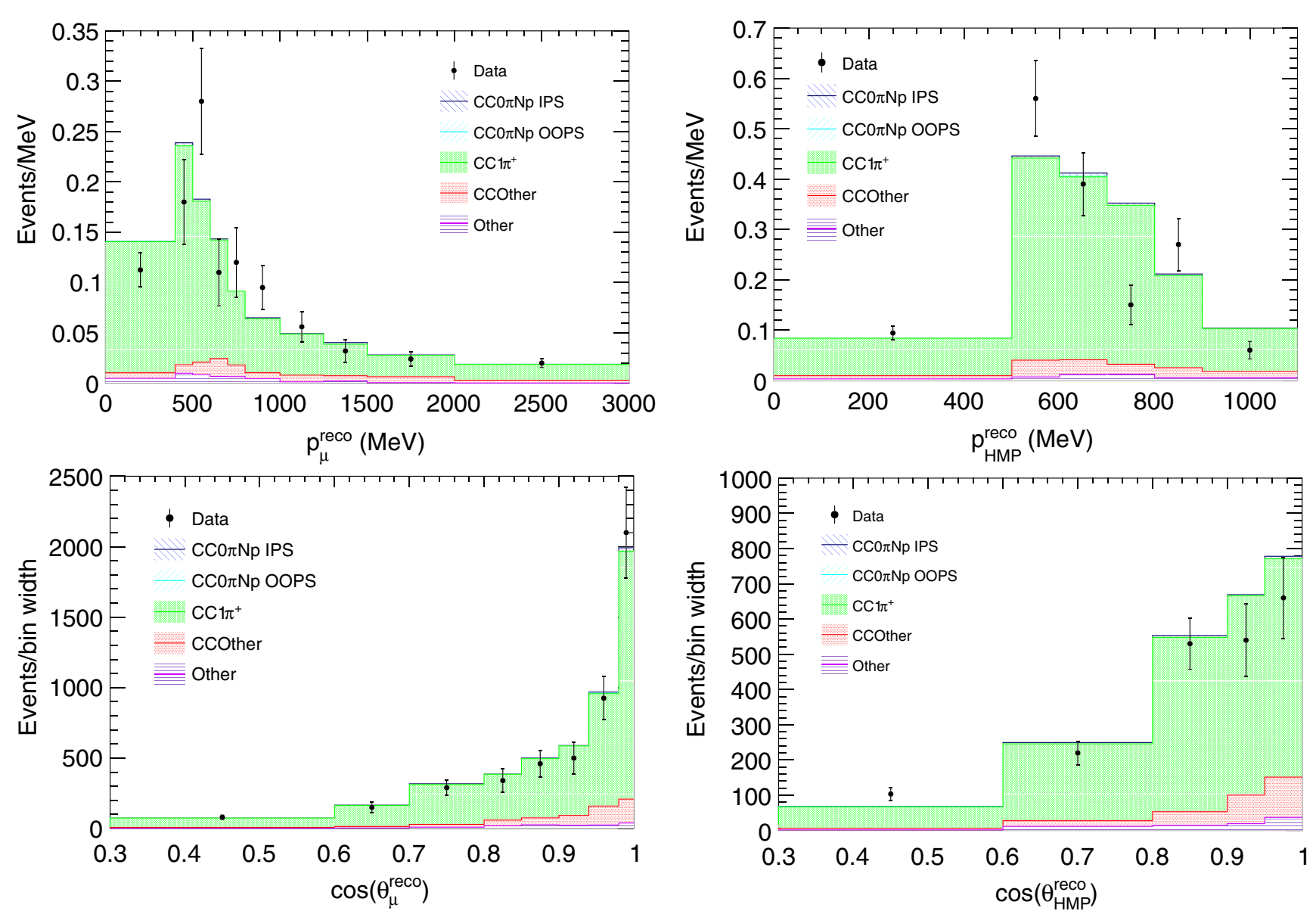

FIG. 11. The reconstructed kinematics of the muon and of the highest momentum positive (HMP) hadron for events selected within the Michel electron tagged control region from both data and NEUT 5.3.2. The plots are broken down by interaction topology and the $\mathrm{CC} 0 \pi$ contribution is broken down by whether the true kinematics of the events adhere to the phase-space constraints (IPS) for the inferred kinematics analysis or fall outside of them (OOPS).

of target nucleons and the bin width before being efficiency corrected to produce a differential cross section.

Such a method of deconvolution is entirely unregularized and is therefore equivalent to using D'Agostini iterative unfolding [56] with an infinite number of iterations or to simply inverting the detector response matrix providing this gives an entirely positive unsmeared spectrum. Provided that the analysis bins do not integrate over regions of phase space of rapidly changing efficiency, this method of unsmearing is completely unbiased but is susceptible to the so-called "illposed problem" of deconvolution-where relatively small statistical fluctuations in the reconstructed bins can cause large variations in the fitted contents of true kinematic bins [87]. These results are fully correct and perfectly suitable for further use, for example in fits to constrain parameters in model predictions or to compare the suitability of different models, but they cannot easily be interpreted "by-eye," since they often contain large anticorrelation between adjacent bins which causes the result to strongly "oscillate" between such bins. Moreover, within the pertinent observables in these analyses, neutrino-interaction cross sections are not expected to follow such an oscillating behavior. These large variations between neighboring bins can be suppressed by regularizing the results, i.e., imposing smoothness of the fitted parameters $c_{i}$, thus inducing a small overall reduction of the uncertainties and some dependence of the results on the input signal simulation model. As such, the STV analysis provides both regularized and unregularized results. To achieve this, a regularization term is optionally added to the likelihood in Eq. (10):

$$
-2 \log (L)_{\text {reg }}=p_{\text {reg }} \sum_{i}^{\text {true bins }-1}\left(c_{i}-c_{i+1}\right)^{2}
$$

Here $c_{i}$ is the signal weight for the $i^{\text {th }}$ true bin and $p_{\text {reg }}$ controls the regularization strength. It is clear that this implementation of regularization adds a constraint which can bias the fit toward the shape of the signal model in the input simulation. However, the impact of the bias can be mitigated by the careful selection of an appropriate regularization strength. A simple method of choosing $p_{\text {reg }}$ in such a regularization scheme is the 'L-curve' technique presented in Ref. [88]. In this approach a compromise is found between 
the impact of the regularization (defined by the normalized regularization penalty: $\left.-2 \log (L)_{\text {reg }} / p_{\text {reg }}\right)$ and the goodness of fit (decreased $\log (L)_{\text {reg }}$ ). One of the significant advantages of this method, over those typically used to choose the regularization strength (like tuning the number of iterations) in iterative unfolding methods, is that it is "data-driven": the regularization strength is determined from assessing the properties of real data and is not solely reliant on simulation studies.

It is important to emphasize that the application of regularization produces a result that is easier to interpret without statistical methods but is at least slightly biased. A regularized result is therefore particularly well suited for result-theory comparison plots but the unregularized result is likely more suitable for forming quantitative conclusions. For this reason unregularized results will be provided in both the multidifferential and STV analyses.

\section{Iterative D'Agostini unfolding}

Unfolding accounts for smearing between the true spectrum and reconstructed spectrum due to the detector efficiency and resolution. The relation between true and measured spectrum can be written as

$$
E_{j}=\sum_{i=1}^{N_{t}} S_{j i} C_{i},
$$

where $C_{i}$ is a number of events in true bin $i, E_{j}$ is a number of events in measured bin $j, S_{j i}$ is a smearing matrix, and $N_{t}$ is the number of true bins.

The smearing matrix is constructed from $\mathrm{MC}$ predictions which gives the information of event migrations. The Iterative unfolding, proposed by D'Agostini [56,57], uses Bayes' theorem to obtain an unsmearing matrix from the smearing matrix as

$$
U_{i j}=\frac{\mathrm{P}_{\text {eff }}\left(E_{j} \mid C_{i}\right) \mathrm{P}_{0}\left(C_{i}\right)}{\sum_{i=1}^{N_{t}} \mathrm{P}\left(E_{j} \mid C_{i}\right) \mathrm{P}_{0}\left(C_{i}\right)},
$$

where $\mathrm{P}\left(E_{j} \mid C_{i}\right)$ is a probability of the true events in bin $i$ measured in bin $j$ written as

$$
\mathrm{P}\left(E_{j} \mid C_{i}\right)=\frac{N_{j i}}{C_{i}},
$$

where $N_{j i}$ is the number of true events in bin $i$ measured in bin $j . \mathrm{P}_{\mathrm{eff}}\left(E_{j} \mid C_{i}\right)$ is defined as:

$$
\mathrm{P}_{\mathrm{eff}}\left(E_{j} \mid C_{i}\right)=\frac{\frac{N_{j i}}{C_{i}}}{\sum_{j=1}^{N_{m}} \frac{N_{j i}}{C_{i}}},
$$

where $N_{m}$ is number of measured bins.
$\mathrm{P}_{0}\left(C_{i}\right)$ is a prior probability representing the predicted number of events in bin $i$, written as

$$
\mathrm{P}_{0}\left(C_{i}\right)=\frac{C_{i}}{\sum_{i=1}^{N_{t}} C_{i}}
$$

Therefore, the unfolded spectrum is

$$
C_{i}^{\prime}=\sum_{j=1}^{N_{m}} U_{i j} E_{j}^{\mathrm{data}},
$$

where $N_{m}$ is the number of bins of measured spectrum. After each iteration, $\mathrm{P}_{0}\left(C_{i}\right)$ is updated with the posterior of the previous iteration.

This method is regularized by choosing the number of iterations, inducing a bias toward the input simulation used. Such bias is tested through multiple mock data sets with alternative simulation models. The number of iterations was chosen by requiring the $\chi^{2}$ values obtained between the unfolded result and the truth of these mock data sets to reach a stable value: 2 -iterations for $\Delta p_{p}, 6$-iterations for $\Delta \theta_{p}$ and 4-iterations for $\left|\Delta \vec{p}_{p}\right|$. The bias in the results was shown to always be well within the uncertainties.

Overall this produces an efficiency corrected and unfolded distribution of signal events which must then account for the flux normalization, the number of target nucleons and the bin width to form a differential cross section, as described by Eq. (9).

\section{E. Multidifferential muon and proton kinematics}

This analysis measures the multidifferential cross section of $\mathrm{CC} 0 \pi$ events as a function of the muon and proton kinematics and the proton multiplicity. As previously described, a multidimensional efficiency correction is applied, the cross section is evaluated with a binned likelihood fit and the background is constrained by using dedicated control regions. The binning, reported in Table III, is chosen to keep the systematic uncertainty smaller than the statistical uncertainty and to cope with the track reconstruction capabilities of the detector. Due to the small available statistics, the events with two or more protons are all collected in a single bin.

The statistical uncertainties are evaluated by fluctuating the total number of observed event in each bin with a Poisson probability and running the fit multiple times. The systematic uncertainties are evaluated by running the analysis on many toy data sets produced by varying the parameters describing the systematics effects detailed in Sec. IV C. The uncertainties are then found by computing the covariance of the resultant cross sections between every pair of analysis bins. The fractional uncertainties are shown in Fig. 12 for some representative bins. The different sources of systematic uncertainties are shown separately and the total systematic uncertainty is evaluated by simultaneously varying all the nuisance parameters corresponding to the different source of 
TABLE III. Bins in muon and proton kinematics in which the multidifferential cross section is measured for the 0 proton sample (top) and the 1 proton sample (bottom).

\begin{tabular}{|c|c|c|}
\hline $\cos \theta_{\mu}$ & & $p_{\mu}(\mathrm{GeV})$ \\
\hline \multicolumn{3}{|l|}{$-1.0,-0.3$} \\
\hline$-0.3,0.3$ & & \\
\hline $0.3,0.6$ & & $\begin{aligned} 0.0,0.3,0.4,30 \\
0.0,0.3,0.4,0.5,0.6,30\end{aligned}$ \\
\hline $0.6,0.7$ & & \\
\hline $0.7,0.8$ & & $0.0,0.3,0.4,0.5,0.6,0.7,0.8,30$ \\
\hline $0.8,0.85$ & & $0.0,0.4,0.5,0.6,0.7,0.8,30$ \\
\hline $0.85,0.9$ & & \multirow{4}{*}{$\begin{array}{c}0.0,0.3,0.4,0.5,0.6,0.7,0.8,1.0,30 \\
0.0,0.4,0.5,0.6,0.7,0.8,1.25,30 \\
0.0,0.4,0.5,0.6,0.7,0.8,1.0,1.25,1.5,2.0,30 \\
0.0,0.5,0.65,0.8,1.25,2.0,3.0,5.0,30\end{array}$} \\
\hline $0.9,0.94$ & & \\
\hline $0.94,0.98$ & & \\
\hline $0.98,1.0$ & & \\
\hline $\cos \theta_{\mu}$ & $\cos \theta_{p}$ & $p_{p}(\mathrm{GeV})$ \\
\hline \multirow{4}{*}{$\begin{array}{l}-1.0,-0.3 \\
-0.3,0.3\end{array}$} & $-1.0,0.87,0.94,0.97,1.0$ & \\
\hline & $-1.0,0.75,0.85$ & \\
\hline & $0.85,0.94$ & $0.5,0.68,0.78,0.9,30$ \\
\hline & $0.94,1.0$ & \\
\hline \multirow[t]{3}{*}{$0.3,0.8$} & $-1.0,0.3,0.5$ & \\
\hline & $0.5,0.8$ & $0.5,0.6,0.7,0.8,0.9,30$ \\
\hline & $0.8,1.0$ & $0.5,0.6,0.7,0.8,1.0,30$ \\
\hline \multirow[t]{3}{*}{$0.8,1.0$} & $-1.0,0.0,0.3$ & \\
\hline & $0.3,0.8$ & $0.5,0.6,0.7,0.8,0.9,1.1,30$ \\
\hline & $0.8,1.0$ & \\
\hline
\end{tabular}

uncertainties. The flux uncertainty is the largest, followed by detector effects. The background modeling uncertainty is sizeable, i.e., of the same order of detector effects, only in the regions with high momentum forward going muons where the background is larger. Finally the signal modeling uncertainty is only non-negligible in the region of backward, low momentum muons where the detector reconstruction capabilities are limited and, due to low available statistics, the angular bin is large, averaging over angles with different reconstruction efficiencies. This is also the region where the backgrounds coming from outside the FGD1 fiducial volume are larger. All these effects tend to increase the dependence of the results on the signal modeling in this particular region of phase space. The statistical uncertainty dominates in most of the bins, except in the regions where the width of the bins is driven by the detector performances. For instance the bin of low proton momentum cannot be further subdivided due to the limited resolution for short tracks. Analogously, in the regions where the muon or the proton have an angle almost perpendicular to the neutrino direction, in order to match the reconstruction capabilities of the detector in absence of a TPC track, the measurement is reported in large bins, and thus systematic uncertainties are larger than statistical uncertainty. Figure 12 shows the uncertainties on the measurement of proton multiplicity. In this case, integrating over all the bins of muon and proton kinematics, the statistical uncertainty is always smaller than the systematic ones. The dominant uncertainty is still due to the flux, followed by the detector effects, which become very important in the events with two or more protons where the outgoing nucleons have very low momentum and are thus difficult to reconstruct. The uncertainty due to background is completely negligible, while the effect of nucleon FSI becomes important at increasing proton multiplicity due to migration effects between different multiplicity bins. The uncertainty due to signal modeling is very small and more or less constant across all the multiplicities.

The extracted $\mathrm{CC} 0 \pi$ multidifferential cross-section results are shown in Figs. 13 and 14, compared to a variety of different model predictions. Value of the comparisons' $\chi^{2}$ are reported in the figures. Additional model comparisons to assess the suitability of RFG + RPA nuclear models (Figs. 24-25) and the impact of FSI (Figs. 26-27) are also shown in Appendix A 1. The last bin of each of the momenta bins is shortened to improve the plot's readability but these bins are normalized by their total width (as specified in Table III) in accordance with Eq. (9). These results are discussed in details in Sec. V.

The total $\mathrm{CC} 0 \pi$ cross section extracted is given in Table IV alongside a prediction from the default NuWro 11q simulation (which will be used as a standard reference for the comparison of the integrated measured cross section in all the analyses).

\section{F. Single Transverse Variables}

In this analysis, a novel set of observables is used to directly probe nuclear effects in measurements of 

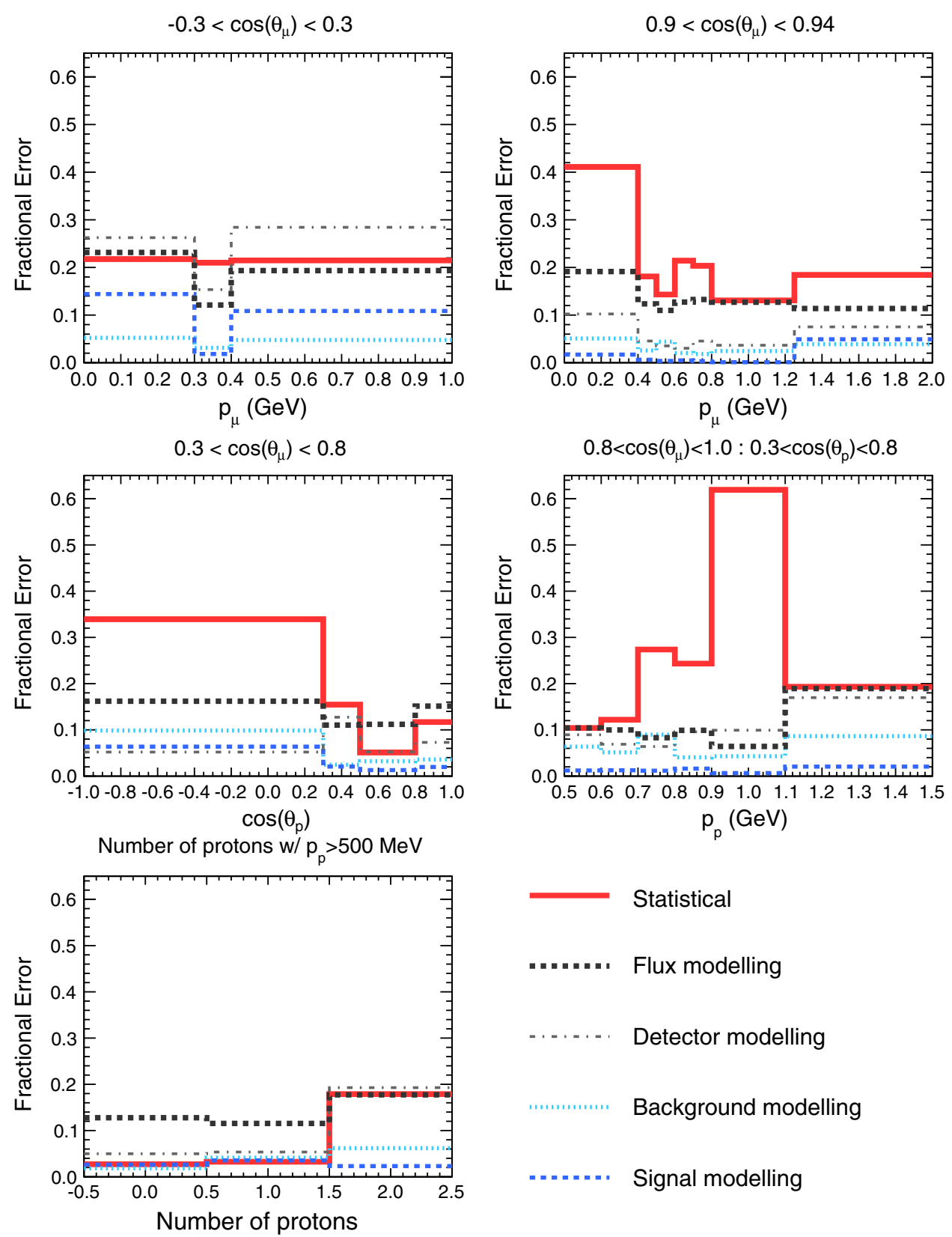

FIG. 12. Statistical and systematic uncertainty (separated by source) within some representative bins of the multidifferential analysis.

$\mathrm{CC} 0 \pi+\mathrm{Np}$ interactions. As previously discussed, these observables exploit the kinematic imbalance between the outgoing lepton and highest momentum proton in the plane transverse to the incoming neutrino, which can act as a powerful probe of nuclear effects both in the initial state and in FSI.

To extract the $\mathrm{CC} 0 \pi+\mathrm{Np}$ differential cross section in the STV, a binned likelihood fit to the number of selected events in reconstructed STV bins is used, as described in Sec. IV D. The uncertainties are evaluated from the postfit covariance matrix, thereby assuming they are Gaussian distributed. The binning for each of the STV are shown in Table V but it should be noted that these STV bins are also restricted to the reduced muon and proton kinematic phase space discussed in Sec. III. The binning is chosen such that the statistical error is comparable to the systematic error and that the bin widths are comparable to the detector resolution.

As described in Sec. IV D, the fit must include effects from plausible variation of detector, flux, and neutrino interaction models. This is achieved by fitting the systematic parameters described in Sec. IV C alongside the signal weights in both the signal region and control samples simultaneously [as described in Eq. (13)]. The systematic uncertainty due to the impact of these parameters is assessed alongside the statistical uncertainty from a 

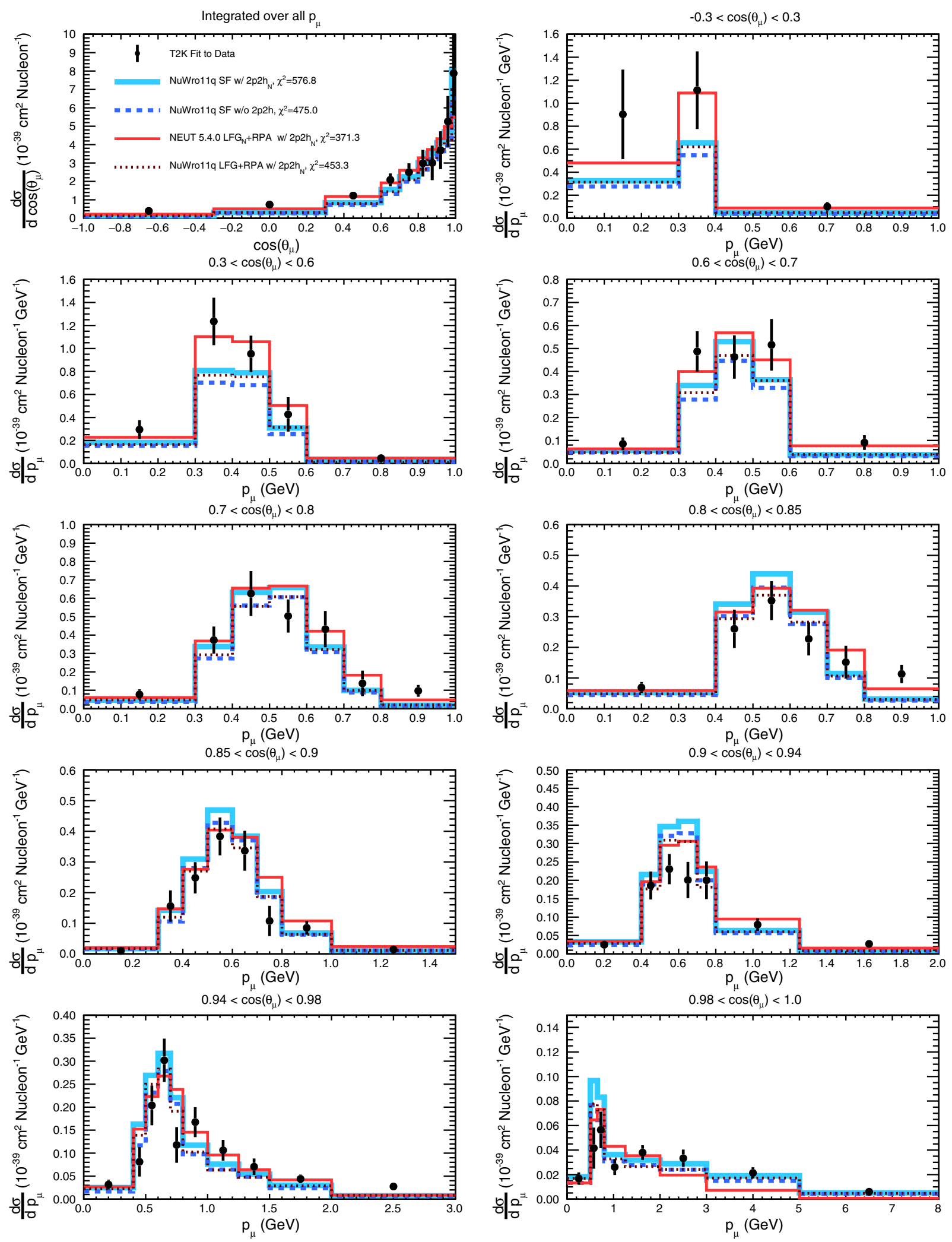

FIG. 13. Measurement of the cross section as a function of the muon kinematics when there are no protons (with momenta above $500 \mathrm{MeV}$ ). The data are compared to: NuWro 11q with the SF nuclear model, both with and without additional 2p2h contribution; NEUT 5.4.0, which uses an LFG + RPA model that includes $2 \mathrm{p} 2 \mathrm{~h}$ predictions; and NuWro 11q with an LFG + RPA nuclear model and a separate $2 \mathrm{p} 2 \mathrm{~h}$ prediction. $2 p 2 h_{N}$ indicates the $2 \mathrm{p} 2 \mathrm{~h}$ model is an implementation of the Nieves et al. model of Ref. [78]. The ' $\mathrm{N}$ ' subscript after LFG indicates that the model is using both a $1 \mathrm{p} 1 \mathrm{~h}$ and $2 \mathrm{p} 2 \mathrm{~h}$ prediction from the aforementioned model of Nieves et al. More details of these models can be found in Sec. IV A. Note that the last momentum bin in each plot is shortened for readability. 

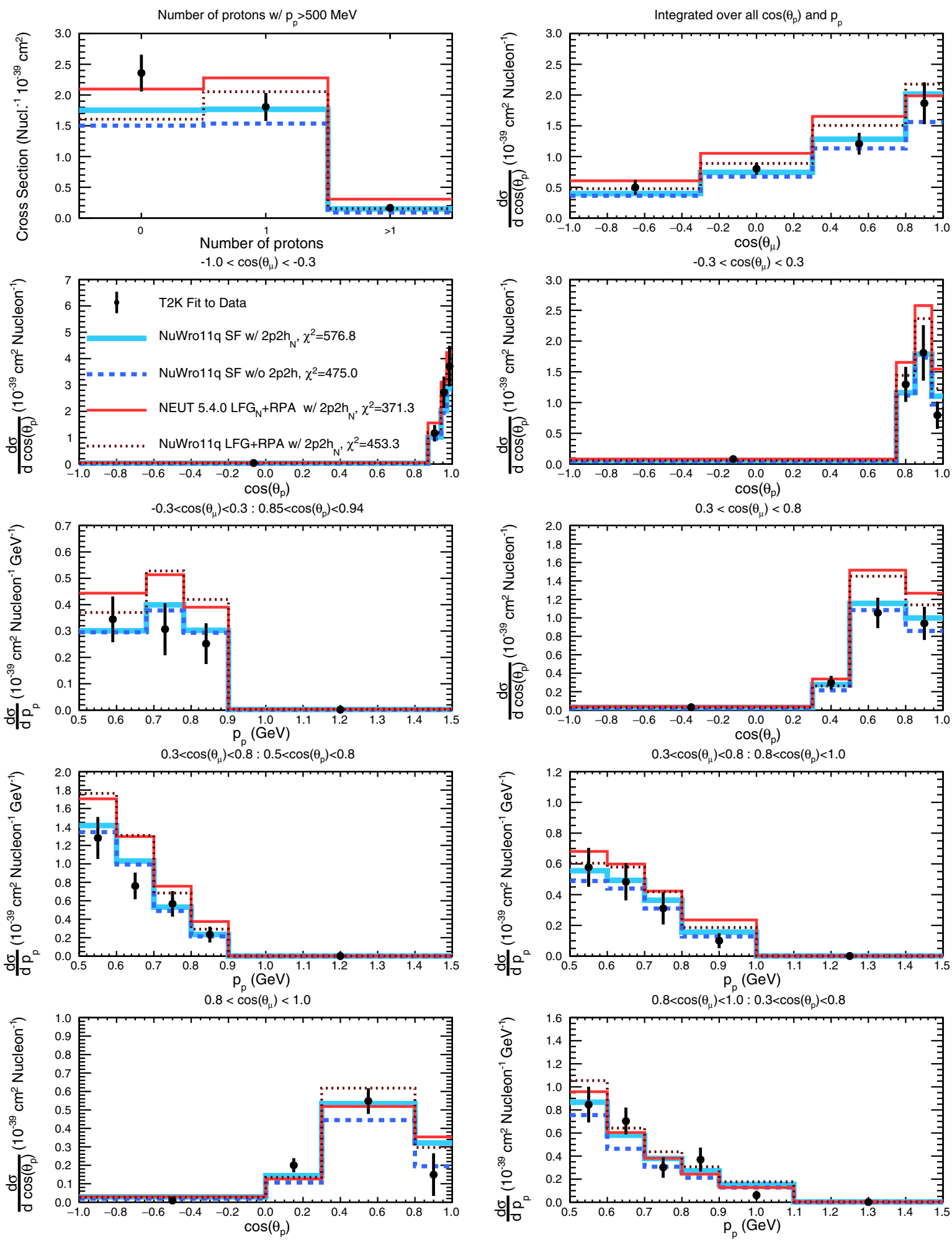

FIG. 14. Measurement of the cross section as a function of the proton multiplicity (top left) and as a function of proton and muon kinematics where there is exactly one proton (with momentum above $500 \mathrm{MeV}$ ). The data are compared to: NuWro 11q with the SF nuclear model, both with and without an additional 2p2h contribution; NEUT 5.4.0, which uses an LFG + RPA model that includes $2 \mathrm{p} 2 \mathrm{~h}$ predictions; and NuWro $11 \mathrm{q}$ with an LFG + RPA nuclear model and a separate $2 \mathrm{p} 2 \mathrm{~h}$ prediction. The same models are also compared to the cross section as a function of proton multiplicity. $2 p 2 h_{N}$ indicates the $2 \mathrm{p} 2 \mathrm{~h}$ model is an implementation of the Nieves et al. model of Ref. [78]. The ' $\mathrm{N}$ ' subscript after LFG indicates that the model is using both a $1 \mathrm{p} 1 \mathrm{~h}$ and $2 \mathrm{p} 2 \mathrm{~h}$ prediction from the aforementioned model of Nieves et al. More details of these models can be found in Sec. IV A. Note that the last momentum bin in each plot is shortened for readability. 
TABLE IV. The total $\mathrm{CC} 0 \pi$ cross section extracted in units of $10^{-39} \mathrm{~cm}^{2}$ within the multi-differential analysis alongside the prediction from NuWro 11q using an SF nuclear model and with the $2 \mathrm{p} 2 \mathrm{~h}$ model of Nieves et. al. [78].

\begin{tabular}{lc}
\hline \hline Cross section & NuWro prediction \\
\hline $4.329 \pm 0.502$ & 3.669 \\
\hline \hline
\end{tabular}

TABLE V. The chosen binning for STV cross-section extraction.

\begin{tabular}{lcc}
\hline \hline$\delta p_{\mathrm{T}}(\mathrm{GeV})$ & $\delta \phi_{\mathrm{T}}$ (radians) & $\delta \alpha_{\mathrm{T}}$ (radians) \\
\hline $0.0-0.08$ & $0.0-0.067$ & $0.0-0.47$ \\
$0.08-0.12$ & $0.067-0.14$ & $0.47-1.02$ \\
$0.12-0.155$ & $0.14-0.225$ & $1.02-1.54$ \\
$0.155-0.2$ & $0.225-0.34$ & $1.54-1.98$ \\
$0.2-0.26$ & $0.34-0.52$ & $1.98-2.34$ \\
$0.26-0.36$ & $0.52-0.85$ & $2.34-2.64$ \\
$0.36-0.51$ & $0.85-1.50$ & $2.64-2.89$ \\
$0.51-1.1$ & $1.50-\pi$ & $2.89-\pi$ \\
\hline \hline
\end{tabular}

post-fit covariance matrix of the parameters, which itself is constructed from the shape of the likelihood surface close to the best-fit point. All parameters are then marginalized in order to project the uncertainty onto the true number of selected signal events in bins of the STV. For a more conservative error estimation, the prefit uncertainty on the detector and flux systematic parameters is considered when evaluating the uncertainty on the subsequent flux normalization and efficiency correction. Parameters describing the signal which are overly degenerate with the signal weights (such as $M_{A}^{\mathrm{QE}}$ ) are not fit. In such cases their uncertainty is taken into account, without any constraints from the data, by computing the effect of their variation on the efficiency corrections. Such effect is found to be relatively small (less than $2 \%$ in all but the last bin of $\delta p_{\mathrm{T}}$ and $\delta \phi_{\mathrm{T}}$ where it is about $4 \%$ ). Further details regarding the uncertainty calculation and the handling of systematic uncertainties can be found in Ref. [58].

The extracted cross sections from the regularized fit in each of the STV are compared to the latest predictions from the current state-of-the-art models from the NEUT 5.3.2.2, NEUT 5.4.0, GENIE 2.12.4, NuWro 11q and GiBUU 2016 neutrino interaction simulations in a variety of configurations. The $\chi^{2}$ of each comparison is reported within the figures. As discussed in Sec. IV D 1, it can be more useful to instead consider the $\chi^{2}$ formed from the unregularized result but in this case the conservative datadriven regularization strength means that the difference in the regularized and unregularized $\chi^{2}$ is marginal. This is demonstrated and discussed in Appendix B (which provides the $\chi^{2}$ from the comparisons with the unregularized result).
The left plots of Fig. 15 compare the results to a variety of different initial state models whilst a shape-only comparison to a subset of these shown on the right alongside the GiBUU 2016 prediction. The contribution from each interaction mode predicted from NEUT 5.3.2.2 is shown in Fig. 16, alongside the impact of altering the simulations with and without a $2 \mathrm{p} 2 \mathrm{~h}$ contribution and modifying the FSI strength by varying the mean free path of nucleons within the NEUT FSI cascade model. Finally a similar breakdown by interaction mode is then made for GiBUU and GENIE in Fig. 17. In GENIE the empirical $2 \mathrm{p} 2 \mathrm{~h}$ contribution, used for instance in the neutrinointeraction model of the $\mathrm{NO} \nu \mathrm{A}$ experiment [89], is enabled. Figures to evaluate the impact of RPA and the role of regularization of the cross-section extraction are shown in Appendix A 2. These results are discussed in details in Sec. V.

The total $\mathrm{CC} 0 \pi+\mathrm{Np}$ cross section extracted (within the phase-space constraints listed in Table I) for each of the STV is given in Table VI alongside a prediction from NuWro 11q. These total cross sections are not identical since the best-fit parameters are altered slightly depending on which projection of the event selection is used as an input.

\section{G. Proton inferred kinematics}

As outlined in Sec. III, this analysis uses the inferred kinematic imbalance between measured proton kinematics and what would be inferred from the measured muon kinematics under a QE approximation, which can act as a metric for the extent to which the QE approximation is reliable for events that are approximately characteristic of the dominant sample used in $\mathrm{T} 2 \mathrm{~K}$ neutrino oscillation analyses.

In this analysis, the muon phase space is divided into multiple bins in order to correct for the different selection efficiencies and CC-non-QE contributions across the phase space:

(i) Bin 0: $\cos \theta_{\mu}<-0.6$.

(ii) Bin 1: $-0.6<\cos \theta_{\mu}<0.0 \& p_{\mu}<250 \mathrm{MeV}$.

(iii) Bin 2: $-0.6<\cos \theta_{\mu}<0.0 \& p_{\mu}>250 \mathrm{MeV}$.

(iv) Bin 3: $\cos \theta_{\mu}>0.0 \& p_{\mu}<250 \mathrm{MeV}$.

(v) Bin 4: $0.0<\cos \theta_{\mu}<0.8 \& p_{\mu}>250 \mathrm{MeV}$.

(vi) Bin 5: $0.8<\cos \theta_{\mu} \& 250<p_{\mu}<750 \mathrm{MeV}$.

(vii) Bin 6: $0.8<\cos \theta_{\mu} \& p_{\mu}>750 \mathrm{MeV}$.

Within each muon angular bin, the same binning in the inferred kinematic variables is used, which is shown in Table VII. The binning is chosen to ensure that the efficiency is suitably flat within each bin and that the bin width is not less than the detector resolution.

To estimate the systematic uncertainties the entire unfolding procedure is repeated for a comprehensive set of plausible variations of the T2K reference model according to the systematic uncertainty sources discussed in Sec. IV C. The covariance of the ensemble of results from 

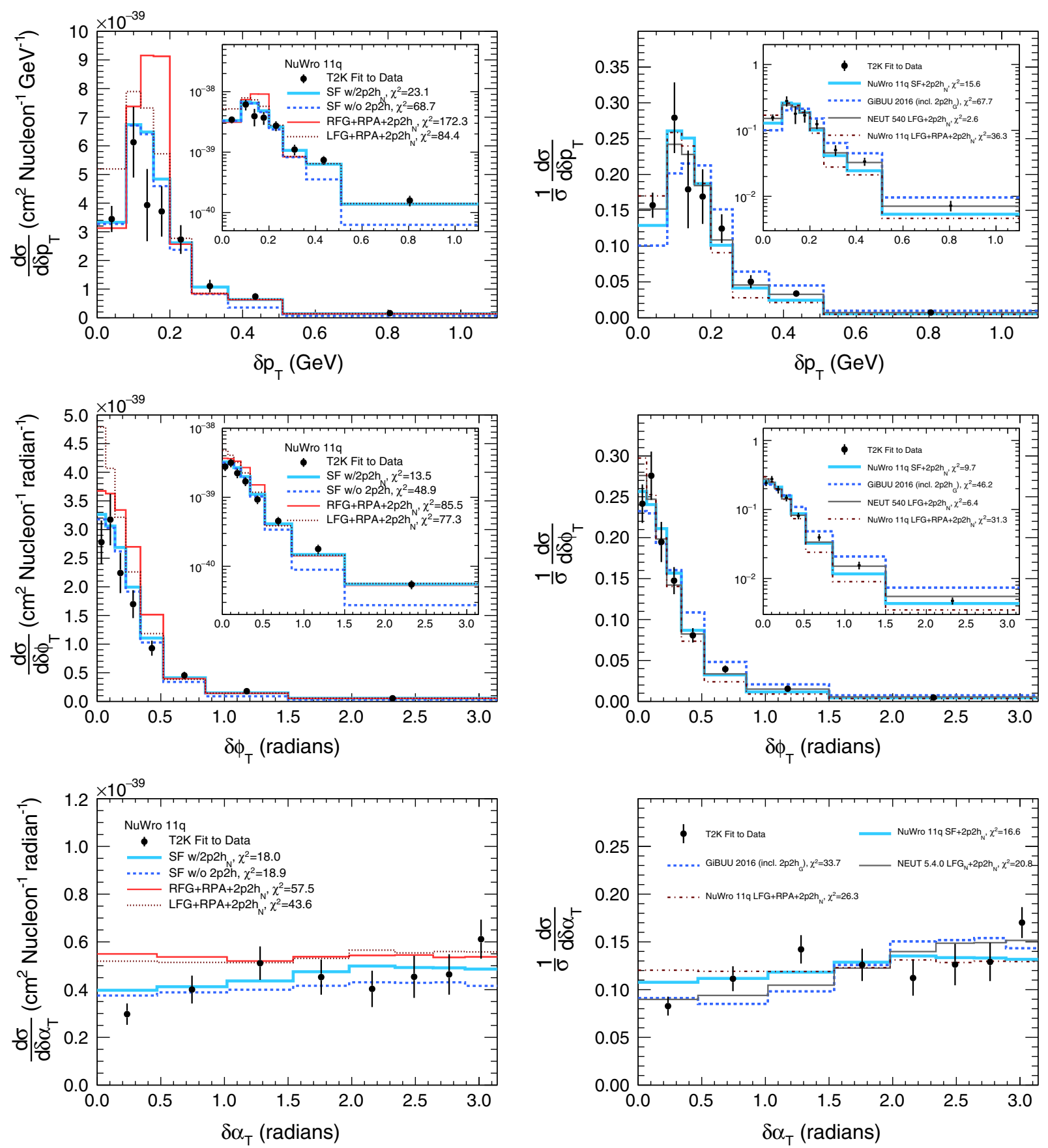

FIG. 15. The extracted differential cross section as a function of the single transverse variables compared to: different initial state models in the NuWro 11q simulation (left); shape only predictions from NuWro 11q and GiBUU 2016 (right). Although it is not shown the NEUT 5.3.2.2 SF prediction has an almost identical shape to the NuWro 11q SF prediction. The NuWro 11q RFG + RPA prediction shown is similar to the NEUT model used as a starting point for T2K's oscillation analyses. $2 p 2 h_{N}$ indicates the Nieves $e t a l$. model of Ref. [78] as implemented in NEUT or NuWro, while $2 p 2 h_{G}$ indicates an extrapolation from electron-scattering data implemented in the GiBUU 2016 simulation [82]. More details of these models can be found in Sec. IV A. The inlays show the same comparisons on a logarithmic scale.

the different pseudoexperiments is then taken to characterize the uncertainty:

$V_{i j}=\frac{1}{N} \sum_{i=1}^{N}\left(\sigma_{i}^{\text {variation }}-\sigma_{i}^{\text {nominal }}\right)\left(\sigma_{j}^{\text {variation }}-\sigma_{j}^{\text {nominal }}\right)$ where $\sigma_{i}^{\text {variation }}$ is an extracted cross section in bin $i$ for a particular variation of the input simulation and $\sigma_{i}^{\text {nominal }}$ is a nominal cross section in bin $i$.

A set of model comparisons, similarly to the one in Sec. IV F and IV E, is shown here for the proton inferred 

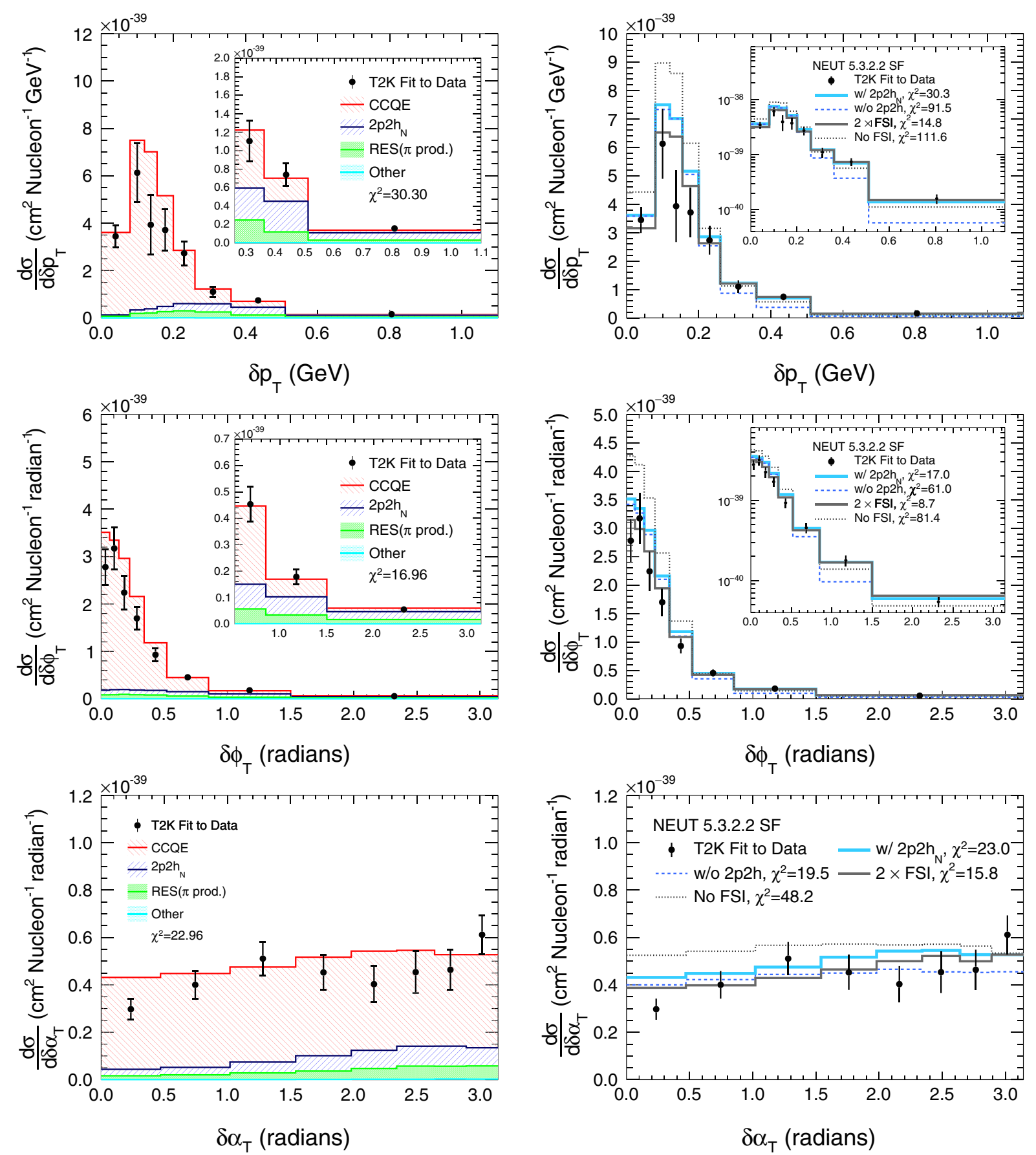

FIG. 16. The extracted differential cross section as a function of the single transverse variables compared to: the NEUT 5.3.2.2 simulation with the SF initial state model and an $a d$ hoc $2 \mathrm{p} 2 \mathrm{~h}$ model (left); the same NEUT simulation with various scalings of the mean free path of nucleons undergoing FSI processes to simulate different FSI strengths (right). $2 p 2 h_{N}$ indicates the Nieves et al. model of Ref. [78] implemented in NEUT. A comparison of the NEUT prediction without a 2p2h contribution is also shown. More details of these models can be found in Sec. IV A. The 'N' subscript after LFG indicates that the model is using both a $1 \mathrm{p} 1 \mathrm{~h}$ and $2 \mathrm{p} 2 \mathrm{~h}$ prediction from the aforementioned model of Nieves et al. The inlays on the left plots show a close-up of the tail regions of $\delta p_{\mathrm{T}}$ and $\delta \phi_{\mathrm{T}}$ whilst those on the right show the same comparisons on a logarithmic scale.

kinematic observables. Figures 18-20 show the results compared to LFG and SF models with and without a $2 \mathrm{p} 2 \mathrm{~h}$ contribution from the NEUT 5.4.0 and NuWro 11q simulations. Figures 21-23 show the impact of altering
FSI strength and removing a (Nieves-like) $2 \mathrm{p} 2 \mathrm{~h}$ contribution within the NEUT 5.3.2.2 simulation. Value of the comparisons $\chi^{2}$ are reported in the figures. Comparisons of the results to RFG nuclear models are shown in 

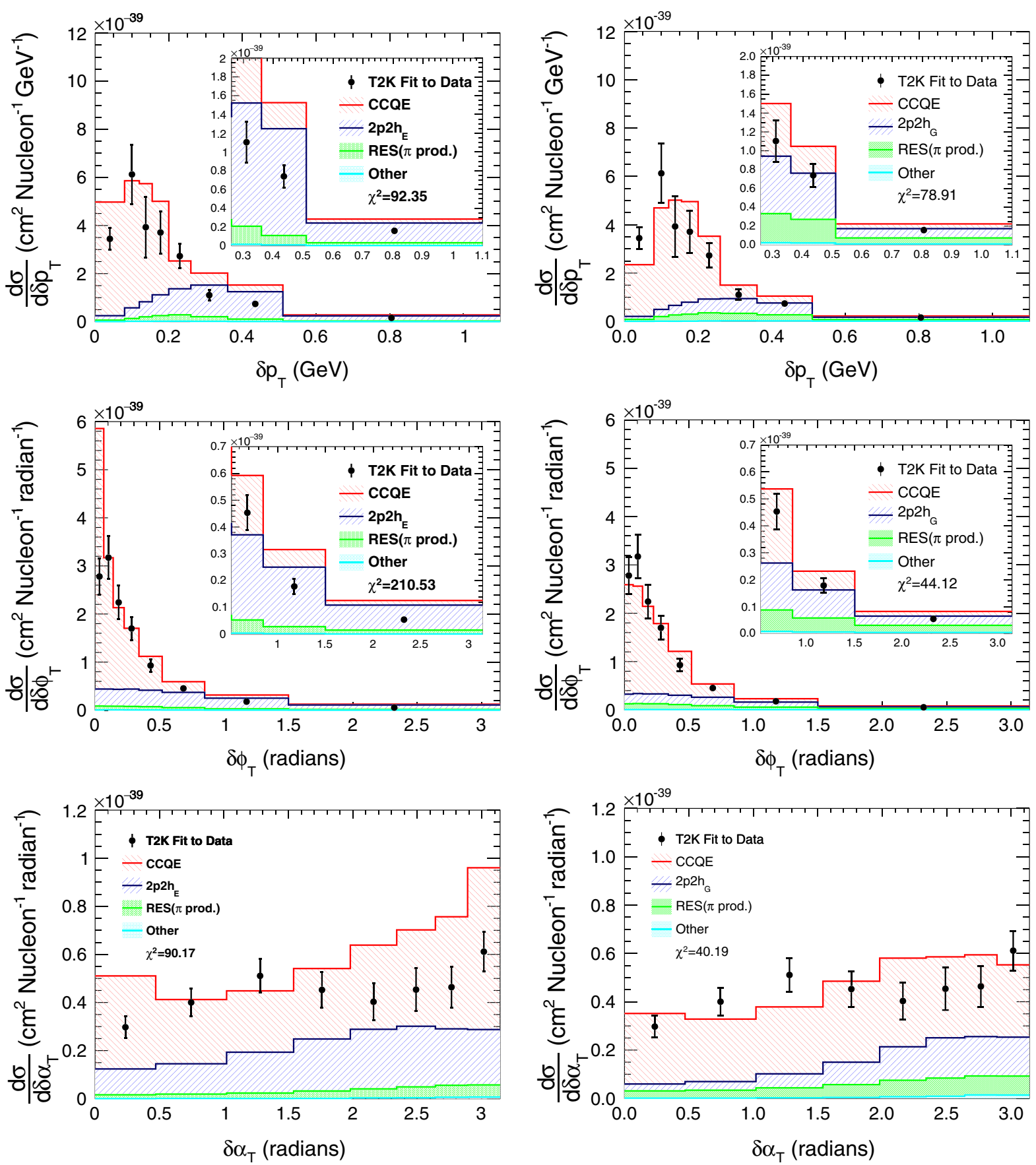

FIG. 17. The extracted differential cross section as a function of the single transverse variables compared to: the GENIE 2.12.4 simulation (left) and the GiBUU 2016 simulation (right). GENIE uses the Bodek and Richie RFG initial state model and this prediction also includes GENIE's empirical $2 \mathrm{p} 2 \mathrm{~h}$ prediction $\left(2 p 2 h_{E}\right)$. This GENIE prediction is similar that used as a starting point for the NO $\nu \mathrm{A}$ experiment's oscillation analyses. More details of these models can be found in Sec. IV A. The inlays on the plots show a close-up of the tail regions of $\delta p_{\mathrm{T}}$ and $\delta \phi_{\mathrm{T}}$.

Appendix A3. These results are discussed in details in Sec. V.

The total $\mathrm{CC} 0 \pi+\mathrm{Np}$ cross section extracted (within the phase-space constraints listed in Table I) for each of the inferred kinematic observables is given in Table VIII alongside a prediction from NuWro 11q. The total cross sections and uncertainties are not identical since the unfolding method couples the systematic parameters between the signal and control regions.

\section{DISCUSSION OF THE RESULTS}

In all of the results shown in Sec. IV $\chi^{2}$ statistics are quoted to indicate the agreement between each model and 
TABLE VI. The total $\mathrm{CC} 0 \pi+\mathrm{Np}$ cross section in units of $10^{-39} \mathrm{~cm}^{2}$ extracted (within the phase space constraints listed in Table I) for each of the STV shown alongside the prediction from NuWro 11q using an SF nuclear model and with the 2p2h model of Nieves et al. [78].

\begin{tabular}{lcc}
\hline \hline Observable & Cross section & NuWro prediction \\
\hline$\delta p_{\mathrm{T}}$ & $1.303 \pm 0.127$ & 1.422 \\
$\delta \phi_{\mathrm{T}}$ & $1.326 \pm 0.124$ & 1.422 \\
$\delta \alpha_{\mathrm{T}}$ & $1.375 \pm 0.130$ & 1.422 \\
\hline \hline
\end{tabular}

TABLE VII. The bins of proton inferred variables in which the cross section is measured.

\begin{tabular}{lcc}
\hline \hline$\Delta p(\mathrm{GeV})$ & $|\Delta p|(\mathrm{GeV})$ & $\Delta \theta$ (degrees) \\
\hline$-5.0,-0.3$ & $0.0,0.3$ & $-360,-5$ \\
$-0.3,0.0$ & $0.3,0.4$ & $-5,5$ \\
$0.0,0.1$ & $0.4,0.5$ & 5,10 \\
$0.1,0.2$ & $0.5,0.6$ & 10,20 \\
$0.2,0.3$ & $0.6,0.7$ & 20,360 \\
$0.3,0.5$ & $0.7,0.9$ & \\
$0.5,5.0$ & $0.9,5.0$ & \\
\hline \hline
\end{tabular}

the result, calculated using the full covariance matrix from the cross-section extraction. However, since no model describes the results well over the entire phase space, these $\chi^{2}$ statistics should be treated carefully: such quantitative estimation of the global data-model agreement is reliable only when a model is capable of describing the whole phase space of the measurement and ideally when it is fit to the result using a well-motivated parametrization of the signal predictions. Moreover, since multiplicative normalization uncertainties make a significant contribution to the covariance of the results, such $\chi^{2}$ statistics can also suffer from Peelle's Pertinent Puzzle [90] and may therefore not accurately characterize the agreement (this does not effect shape-only $\chi^{2}$ ). For this reason these statistics should only be taken as an approximate metric and this section will mainly focus on discussing and interpreting discrepancies between the model and the simulations in specific regions of kinematic phase space, rather than on the overall concurrence indicated by the $\chi^{2}$. However, when doing this it is important to be aware of the significant correlations between each bin in the extracted results. The significant detector smearing in the STV leads to adjacent bins being fairly anticorrelated (up to $\sim 35 \%$, with $\delta p_{T}$ the most affected) whilst the large flux normalization uncertainty correlates all other bins (by $\sim 10 \%$ to $35 \%$ ). Due to the larger regularization strength in the inferred kinematics analysis and the coarser binning in the multidifferential analysis, the anticorrelations are generally much less prominent and most bins are positively correlated by the flux. The full covariance matrices are available in the data release for these results. A summary of the full and shape only $\chi^{2}$ for the regularized and unregularized STV results are provided and discussed in Appendix B.

The measurement of proton multiplicity and proton and muon kinematics from the multidifferential analysis (Figs. 13, 14) shows the phase-space regions where the present models fail to describe the data. From this measurement it can be seen that, when there is no proton above threshold in the final state, while the SF prediction gives a reasonable agreement with the extracted result in the region $(0.6<\cos \theta<0.8)$, it clearly underestimates the cross section in the region of backward muon angle and overestimates it in the region with forward muons for intermediate muon momentum $(0.5-0.8 \mathrm{GeV})$. Conversely, the SF model describes very well the rate of events with one proton above the momentum threshold in the final state, where a slight preference for the presence of $2 \mathrm{p} 2 \mathrm{~h}$ is observed in the region with forward muons $(\cos \theta>0.8)$.

The LFG predictions from NEUT 5.4.0 and NuWro 11q differ when describing events without protons above threshold in the final state in the region with muons at high angle. Here the NEUT implementation describes the results well, while NuWro underestimates the cross section, in a similar manner to its SF prediction. In the phase space with intermediate and low muon angle, both LFG implementations describe the result reasonably well. However, both also overestimate the cross section with one abovethreshold proton in the final state. Indeed, the two LFG models predict a tendency in proton multiplicity to have a larger rate of events with one proton with respect to events without protons, while the extracted result has the opposite behavior.

Finally, in the bin with two or more protons in the final state, the result prefers the SF with $2 \mathrm{p} 2 \mathrm{~h}$ over the case without $2 \mathrm{p} 2 \mathrm{~h}$. Here it can also be seen that the two implementations of LFG give very different predictions, thereby demonstrating the importance of $1 \mathrm{p} 1 \mathrm{~h}$ modeling also for the events with multiple protons.

It is also interesting to compare the effect of FSI and $2 \mathrm{p} 2 \mathrm{~h}$ in these distributions, as shown in Figs. 26-27 in Appendix A 1, where SF with and without $2 \mathrm{p} 2 \mathrm{~h}$ is compared together with different strengths of FSI. A larger FSI strength tends to redistribute events between the bins of proton multiplicity: larger FSI increases the rate of events without protons above the momentum threshold and decreases the number of events with one or more protons above it. On the other hand, $2 \mathrm{p} 2 \mathrm{~h}$ tends to increase the cross section for all proton multiplicities. Since the measurement of the shape of the cross section in proton multiplicity is well known (the uncertainties are dominated by effects that fully correlate all three bins, mostly due to the flux) these results may offer an interesting capability to separate the effects of proton FSI and $2 \mathrm{p} 2 \mathrm{~h}$. However, more robust predictions of the outgoing proton kinematics in $1 \mathrm{p} 1 \mathrm{~h}$ and $2 \mathrm{p} 2 \mathrm{~h}$ events after FSI would be needed in order to exploit the proton multiplicity measurement to expose a possible 

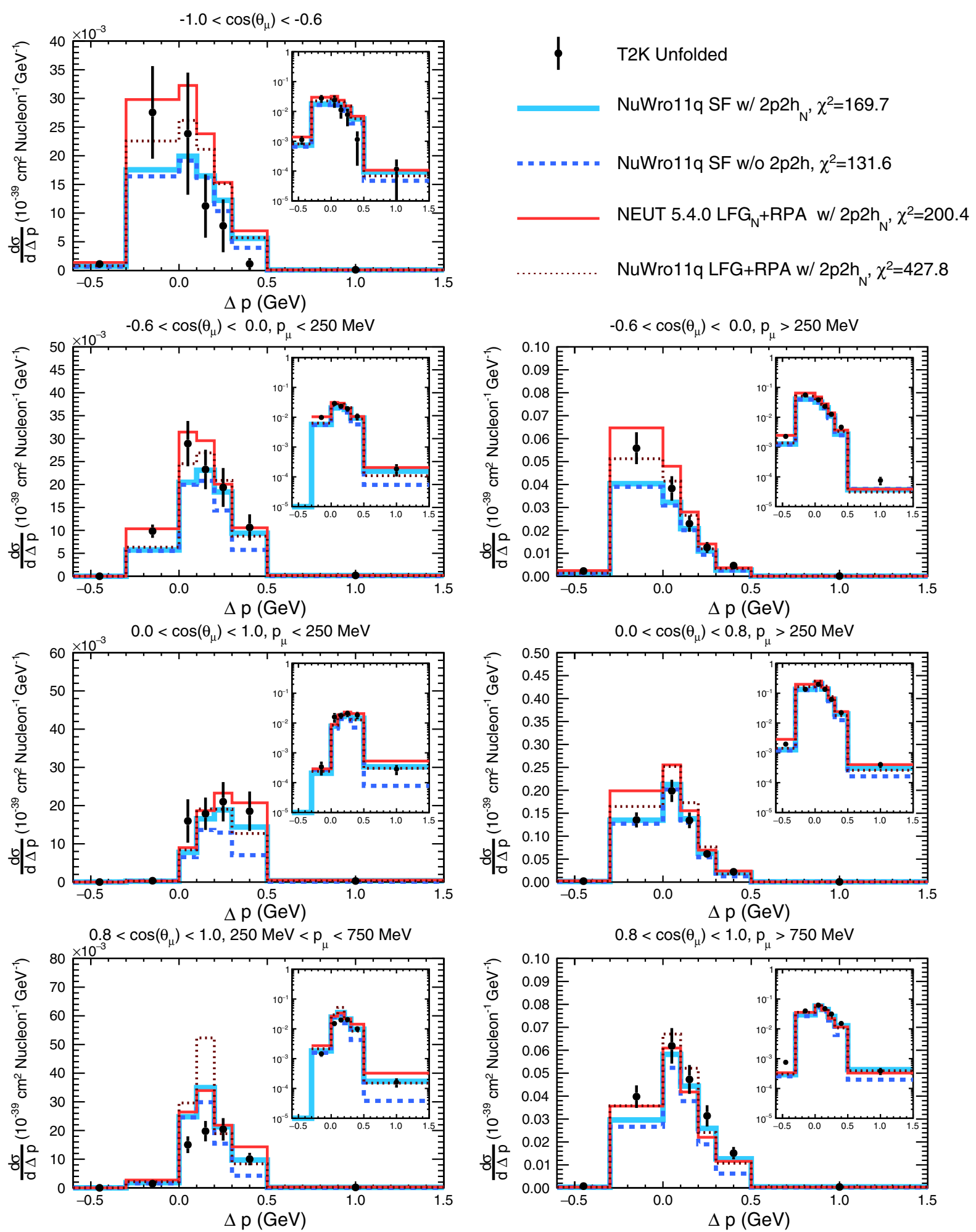

FIG. 18. The extracted differential cross section as a function of the inferred and true proton momentum difference in different muon kinematic bins, within a restricted proton kinematic phase space, compared to a variety of model predictions: NuWro 11q with the SF nuclear model both with and without an additional ad hoc $2 \mathrm{p} 2 \mathrm{~h}$ contribution; NEUT 5.4.0, which uses an LFG + RPA model that includes $2 \mathrm{p} 2 \mathrm{~h}$ predictions; and NuWro $11 \mathrm{q}$ with an LFG + RPA nuclear model and a separate $2 \mathrm{p} 2 \mathrm{~h}$ prediction. $2 p 2 h_{N}$ indicates the $2 \mathrm{p} 2 \mathrm{~h}$ model is an implementation of the Nieves et al. model of Ref. [78]. The ' $\mathrm{N}$ ' subscript after LFG indicates that the model is using both a $1 \mathrm{p} 1 \mathrm{~h}$ and $2 \mathrm{p} 2 \mathrm{~h}$ prediction from the aforementioned model of Nieves et al. More details of these models can be found in Sec. IV A. Note that the first and last bin in each plot is shortened for improved readability. The inlays show the same comparisons on a logarithmic scale. 

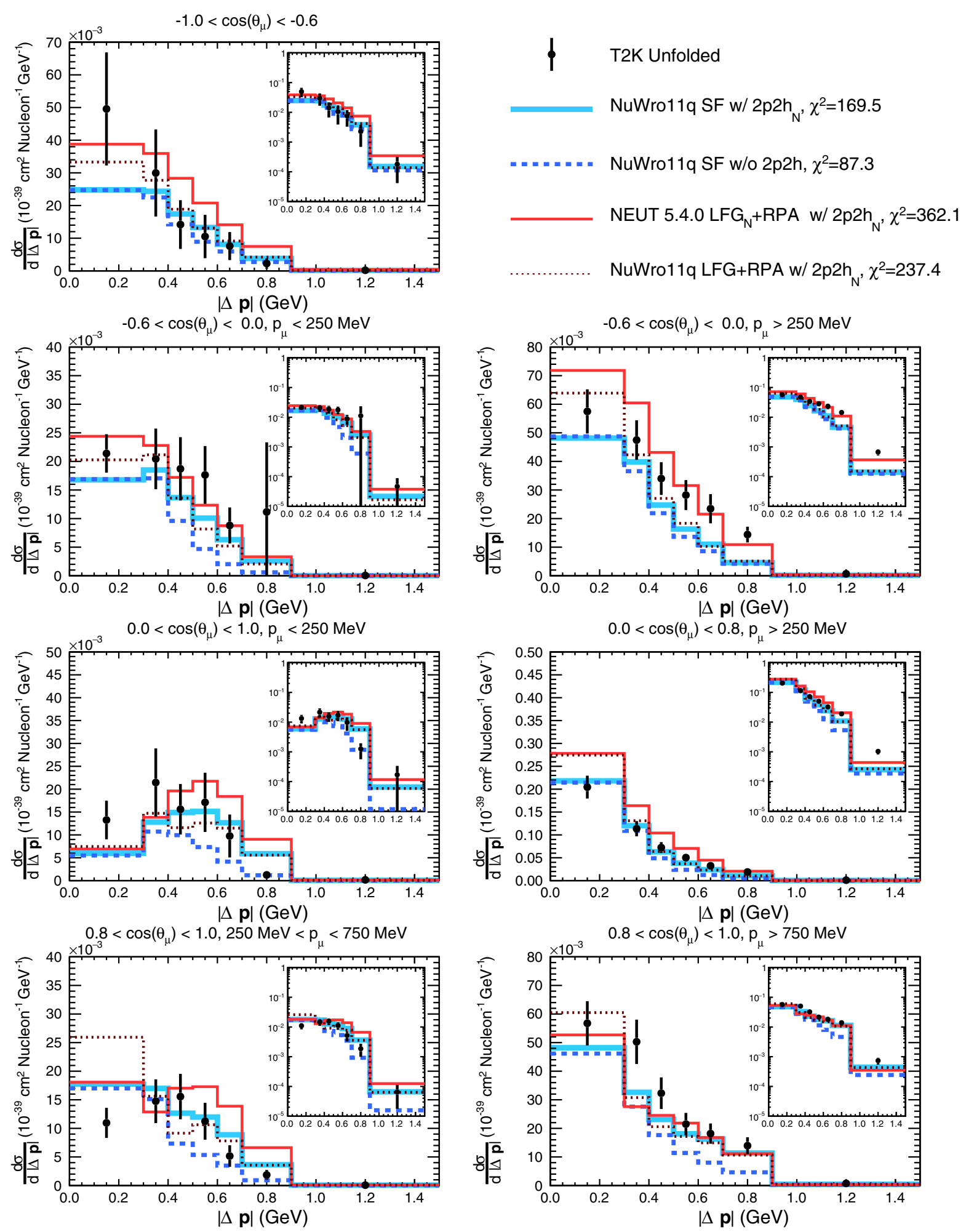

FIG. 19. The extracted differential cross section as a function of the modulus of the inferred and true proton three-momentum difference in different muon kinematic bins, within a restricted proton kinematic phase space, compared to a variety of model predictions: NuWro 11q with the SF nuclear model both with and without an additional ad hoc 2p2h contribution; NEUT 5.4.0, which uses an LFG + RPA model that includes 2p2h predictions; and NuWro 11q with an LFG + RPA nuclear model and a separate 2p2h prediction. $2 p 2 h_{N}$ indicates the $2 \mathrm{p} 2 \mathrm{~h}$ model is an implementation of the Nieves et al. model of Ref. [78]. The ' $\mathrm{N}$ ' subscript after LFG indicates that the model is using both a $1 \mathrm{p} 1 \mathrm{~h}$ and $2 \mathrm{p} 2 \mathrm{~h}$ prediction from the aforementioned model of Nieves et al. More details of these models can be found in Sec. IV A. Note that the last bin in each plot is shortened for improved readability. The inlays show the same comparisons on a logarithmic scale. 

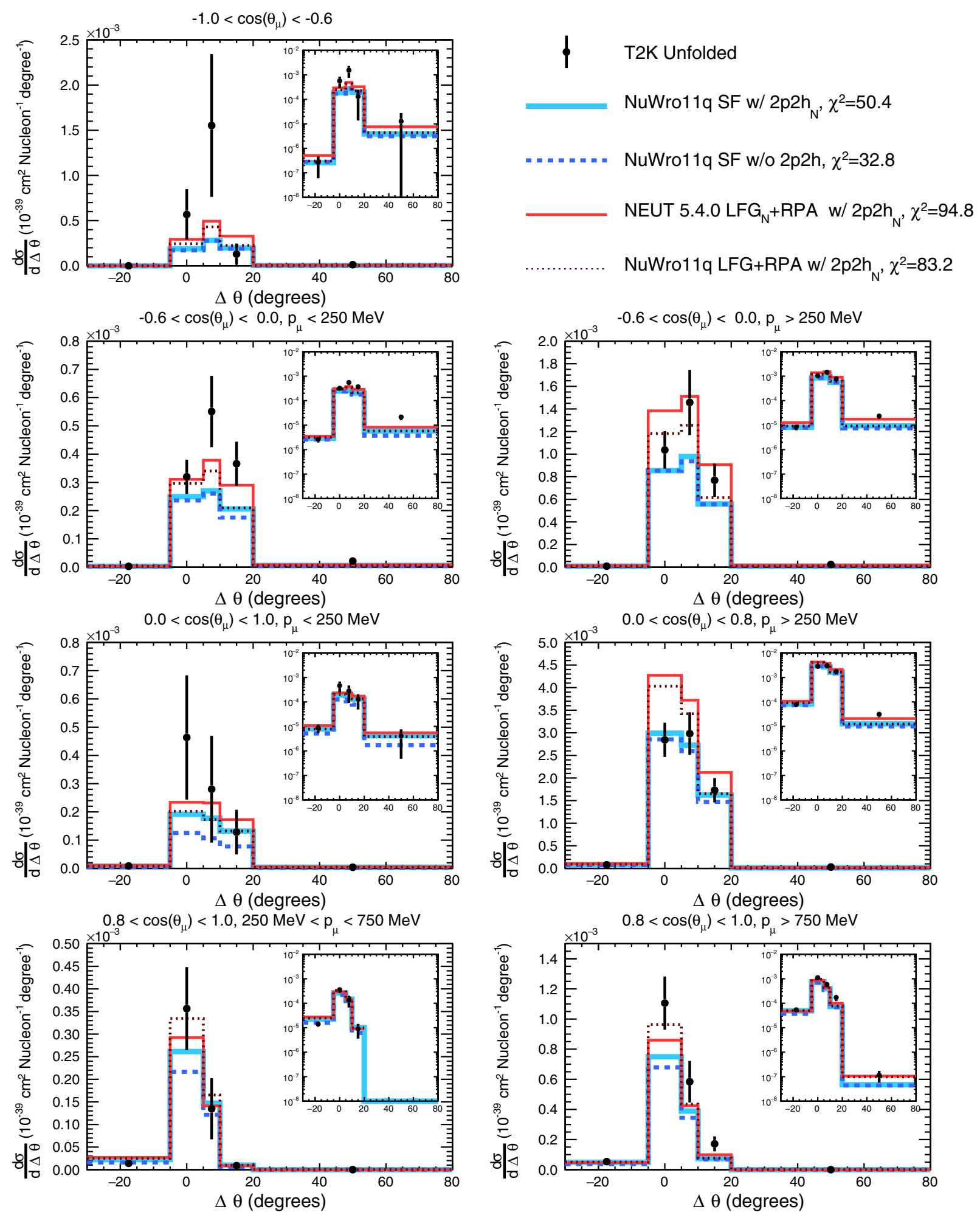

FIG. 20. The extracted differential cross section as a function of the inferred and true proton outgoing-angle difference in different muon kinematic bins, within a restricted proton kinematic phase space, compared to a variety of model predictions: NuWro $11 \mathrm{q}$ with the SF nuclear model both with and without an additional ad hoc $2 \mathrm{p} 2 \mathrm{~h}$ contribution; NEUT 5.4.0, which uses an LFG + RPA model that includes $2 \mathrm{p} 2 \mathrm{~h}$ predictions; and NuWro $11 \mathrm{q}$ with an LFG + RPA nuclear model and a separate $2 \mathrm{p} 2 \mathrm{~h}$ prediction. $2 p 2 h_{N}$ indicates the $2 \mathrm{p} 2 \mathrm{~h}$ model is an implementation of the Nieves et al. model of Ref. [78]. The ' $\mathrm{N}$ ' subscript after LFG indicates that the model is using both a $1 \mathrm{p} 1 \mathrm{~h}$ and $2 \mathrm{p} 2 \mathrm{~h}$ prediction from the aforementioned model of Nieves et al. More details of these models can be found in Sec. IV A. Note that the first and last bin in each plot is shortened for improved readability. The inlays show the same comparisons on a logarithmic scale. 

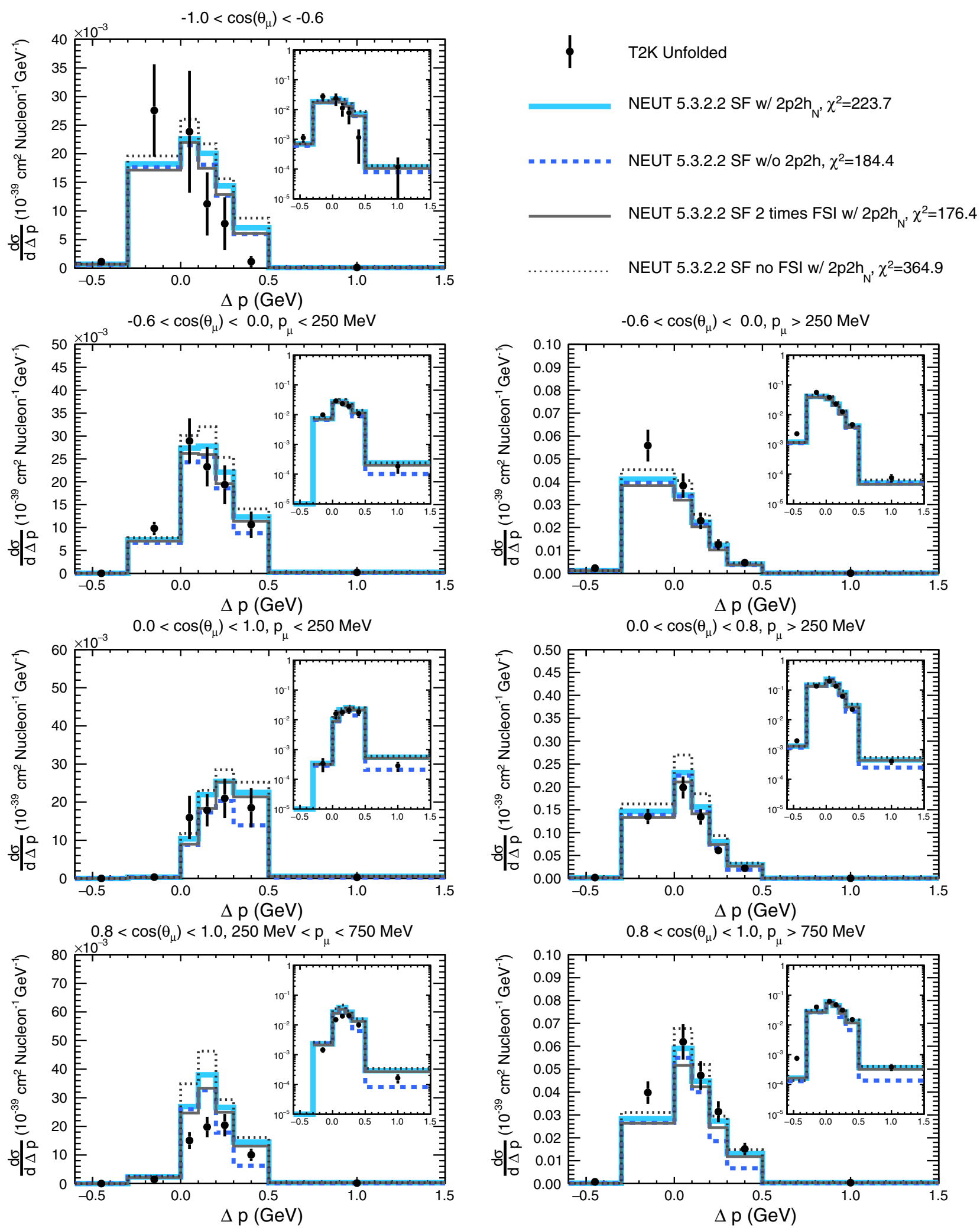

FIG. 21. The extracted differential cross section as a function of the inferred and true proton momentum difference in different muon kinematic bins, within a restricted proton kinematic phase space, compared to the NEUT 5.3.2.2 simulation with various scalings of the mean free path of nucleons undergoing FSI processes to simulate different FSI strengths. A comparison of the NEUT prediction without a $2 \mathrm{p} 2 \mathrm{~h}$ contribution is also shown. $2 p 2 h_{N}$ indicates the $2 \mathrm{p} 2 \mathrm{~h}$ model is an implementation of the Nieves et al. model of Ref. [78]. More details of these models can be found in Sec. IV A. Note that the first and last bin in each plot is shortened for improved readability. The inlays show the same comparisons on a logarithmic scale. 

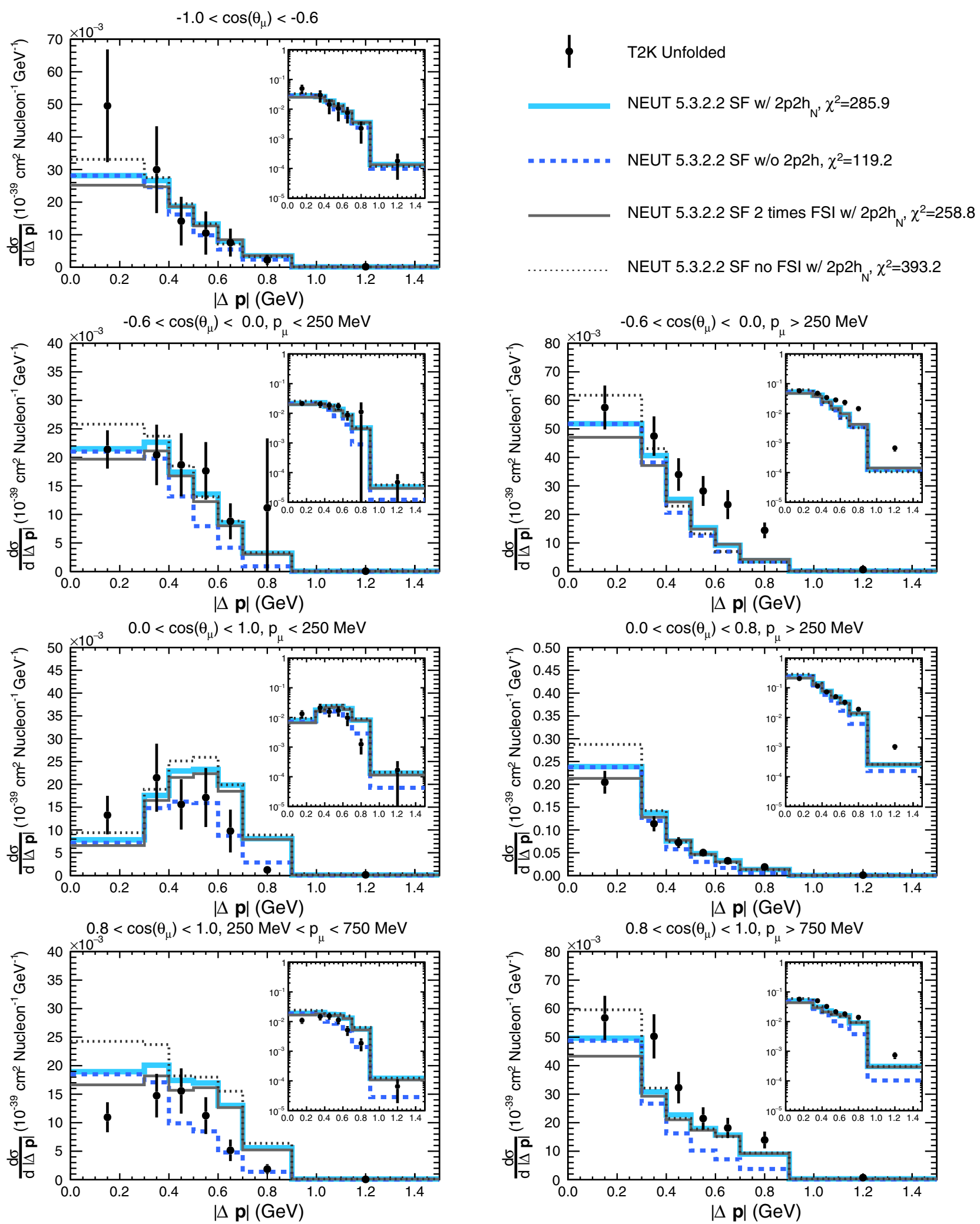

FIG. 22. The extracted differential cross section as a function of the inferred and true proton three-momentum difference in different muon kinematic bins, within a restricted proton kinematic phase space, compared to the NEUT 5.3.2.2 simulation with various scalings of the mean free path of nucleons undergoing FSI processes to simulate different FSI strengths. A comparison of the NEUT prediction without a $2 \mathrm{p} 2 \mathrm{~h}$ contribution is also shown. $2 p 2 h_{N}$ indicates the $2 \mathrm{p} 2 \mathrm{~h}$ model is an implementation of the Nieves et al. model of Ref. [78]. More details of these models can be found in Sec. IV A. Note that the last bin in each plot is shortened for improved readability. The inlays show the same comparisons on a logarithmic scale. 

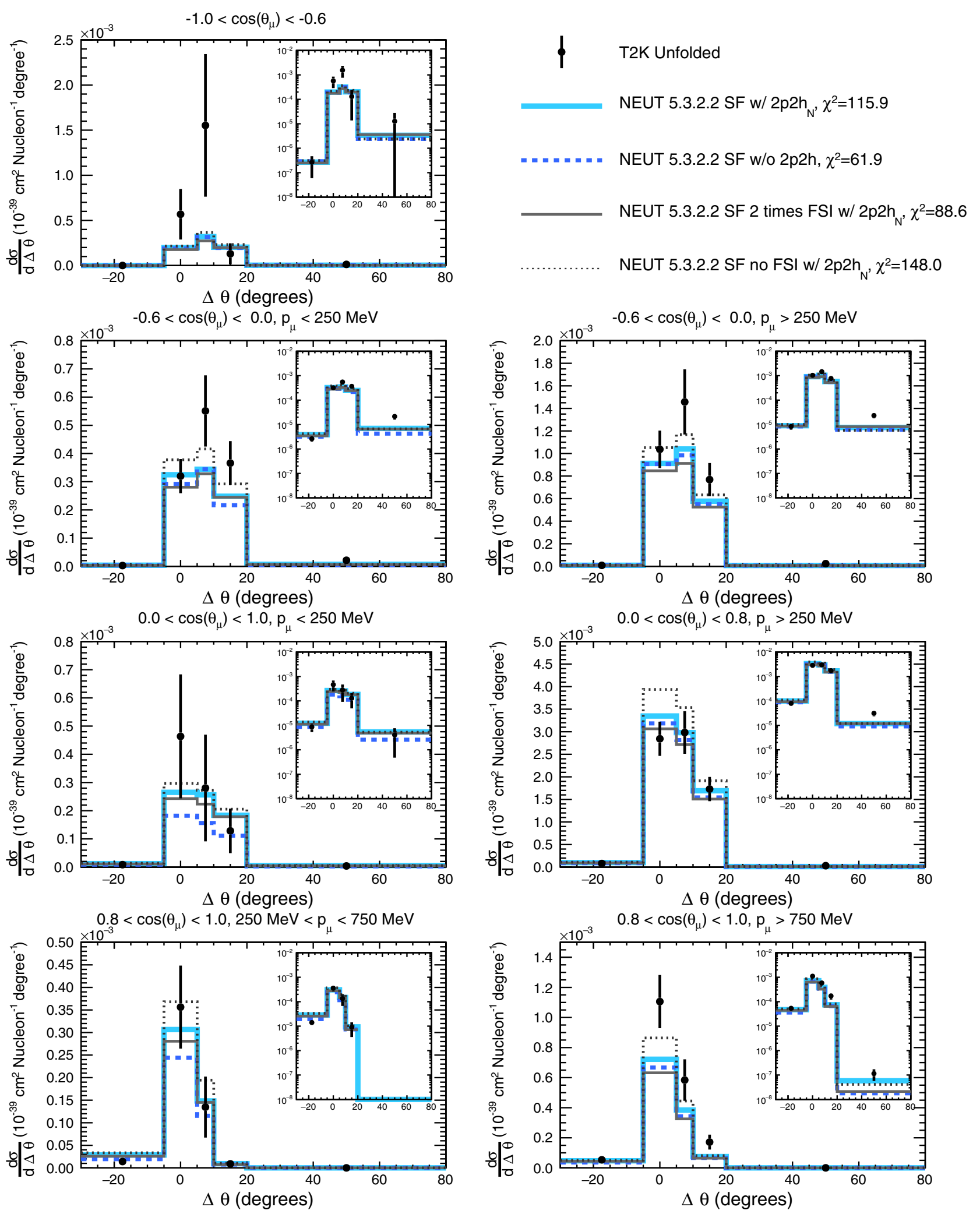

FIG. 23. The extracted differential cross section as a function of the inferred and true proton outgoing-angle difference in different muon kinematic bins, within a restricted proton kinematic phase space, compared to the NEUT 5.3.2.2 simulation with various scalings of the mean free path of nucleons undergoing FSI processes to simulate different FSI strengths. A comparison of the NEUT prediction without a $2 \mathrm{p} 2 \mathrm{~h}$ contribution is also shown. $2 p 2 h_{N}$ indicates the $2 \mathrm{p} 2 \mathrm{~h}$ model is an implementation of the Nieves et al. model of Ref. [78]. More details of these models can be found in Sec. IV A. Note that the first and last bin in each plot is shortened for improved readability. The inlays show the same comparisons on a logarithmic scale. 
TABLE VIII. The total $\mathrm{CC} 0 \pi+\mathrm{Np}$ cross section in units of $10^{-39} \mathrm{~cm}^{2}$ extracted (within the phase space constraints listed in Table I) for each of the inferred kinematic observables alongside the prediction from NuWro 11q using an SF nuclear model and with the $2 \mathrm{p} 2 \mathrm{~h}$ model of Nieves et al. [78].

\begin{tabular}{llc}
\hline \hline Observable & Cross section & NuWro prediction \\
\hline$\Delta p$ & $2.169 \pm 0.235$ & 1.916 \\
$|\Delta p|$ & $2.220 \pm 0.243$ & 1.916 \\
$\Delta \theta$ & $2.247 \pm 0.244$ & 1.916 \\
\hline \hline
\end{tabular}

significant $2 \mathrm{p} 2 \mathrm{~h}$ excess in the result. It is particularly striking that no model is able to simultaneously describe accurately the events with and without above-threshold protons in the final state, the LFG being more in agreement with the result in former and the SF being in better agreement in the latter.

The results are also compared in Figs. 24-25 with RFG models as implemented in NuWro 11q and NEUT 5.3.2.2 and with the RFG with Bodek-Ritchie corrections as implemented in GENIE 2.12.4. GENIE overestimates the cross section in most of the phase space, except for backward-going muons. It also reproduces the same trend as the extracted result in the multiplicity plot, showing more events without a final state proton above the threshold. The other RFG implementations in NuWro and NEUT behave similarly to the NuWro LFG model.

It also is interesting to consider the impact of the RES pion-production contribution to the signal (where the pion is absorbed inside the nucleus). In general, NEUT 5.3.2.2 predicts that the RES contribution to the cross section is always less than about $5 \%$ except when there is one abovethreshold proton in the final state and $\cos \left(\theta_{\mu}\right)>0.8$, where it reaches around $15 \%$, and when there is more than one proton in the final state where the cross section is dominated almost equally be RES and $2 \mathrm{p} 2 \mathrm{~h}$ interactions. Although there is large theoretical uncertainty on this RES contribution (notably from nuclear effects in pion production and in pion FSI), within most bins unrealistically large changes would be required to alter the interpretation of the results.

In general, the interpretation of the aforementioned discrepancies between the result of the multidifferential analysis and different simulations is not straightforward since the measured cross section is affected by multiple initial state and final state nuclear effects which cannot be easily separated in the momentum and angular kinematic distributions. The STV are expressly designed in order to unambiguously distinguish the impact of different nuclear effects, their measurement therefore offers a more transparent interpretation of such discrepancies.

The comparison of the STV distributions to different CCQE models, as implemented in NuWro, are shown in the left plots of Fig. 15. Given the definition of $\delta \vec{p}_{\mathrm{T}}$ [Eq. (2)], the dominant contribution below the Fermi surface $\left(\delta p_{\mathrm{T}} \sim 230 \mathrm{MeV}\right)$ is CCQE with limited FSI strength (as can be seen in the left plots of Fig. 16), thus the Fermi motion determines the bulk structure of $\delta p_{\mathrm{T}}$, thereby allowing it to act as a probe of the initial-state nucleon. The measured $\delta p_{\mathrm{T}}$ distribution strongly disagrees with the RFG prediction: the prominent imprint of the cliff at the Fermi surface, a characteristic of RFG, is firmly disfavored by this result.

It is interesting to note that both Fermi gas models (LFG and RFG) exhibit similar excesses over the result, but at different kinematic regions. Indeed, considering shape-only comparisons of data to various simulations in the right plots of Fig. 15, it can be seen that the LFG predictions well describe the differential distribution, but are plagued by an overestimation of the overall cross section, even if RPA corrections are applied. Such a normalization discrepancy could come from a general overly large CCQE cross section or weak proton FSI keeping too many protons above signal threshold. The latter is particularly further supported in the proton multiplicity plot in Fig. 14, where an increase in proton FSI would migrate events from the 0 proton bin to the 1 proton bin thereby bringing the prediction into better agreement with the results. The fact that the GiBUU (LFG) CCQE prediction in Fig. 17, which largely differs from the NuWro and NEUT LFG models through its FSI modeling, seems to provide a normalization in good agreement with the results adds additional evidence that the normalization discrepancy seen in the NEUT and NuWro LFG normalizations is at least partially related to FSI modeling.

In general it seems that the nucleon dynamics for $\delta p_{\mathrm{T}} \lesssim 400 \mathrm{MeV}$, are better described by SF than Fermi gas models. The consistency between SF and the result at $\delta p_{\mathrm{T}} \sim 300 \mathrm{MeV}$ suggest that the nucleon-nucleon correlations captured by SF are required. Future measurements of the STV with higher statistics may allow further exploration of the nature of such correlations.

Above $\sim 400 \mathrm{MeV}, \delta p_{\mathrm{T}}$ is driven by nucleon-nucleon correlations and FSI effects, so it is not surprising that the predictions from the Fermi gas and SF models become more similar. The SF model in this region, as it is implemented in the simulations, is not fully consistent since a $2 \mathrm{p} 2 \mathrm{~h}$ contribution computed for an LFG model is added on top of the CCQE SF. The SF model without a $2 \mathrm{p} 2 \mathrm{~h}$ contribution is also shown for comparison: within the hard tail of $\delta p_{\mathrm{T}}$ and $\delta \phi_{\mathrm{T}}$ the result clearly indicates the need for additional strength, consistent with that from a $2 \mathrm{p} 2 \mathrm{~h}$ contribution, beyond the nucleon-nucleon correlations already included in the SF model.

Both RFG and LFG models have consistent predictions regarding the total cross section and the $\delta \alpha_{\mathrm{T}}$ distribution, which represents to good approximation the direction of the initial nucleon momentum $\vec{p}_{\mathrm{N}}$. Furthermore, the distributions of $\delta \alpha_{\mathrm{T}}$ show a significant difference between Fermi gas and SF models in the shape. In fact, in NuWro predictions the discrepancy at low $\delta \alpha_{\mathrm{T}}$ between Fermi gas models and the data is caused by RPA (see the left plots of Fig. 28), without which the shape would be consistent. 

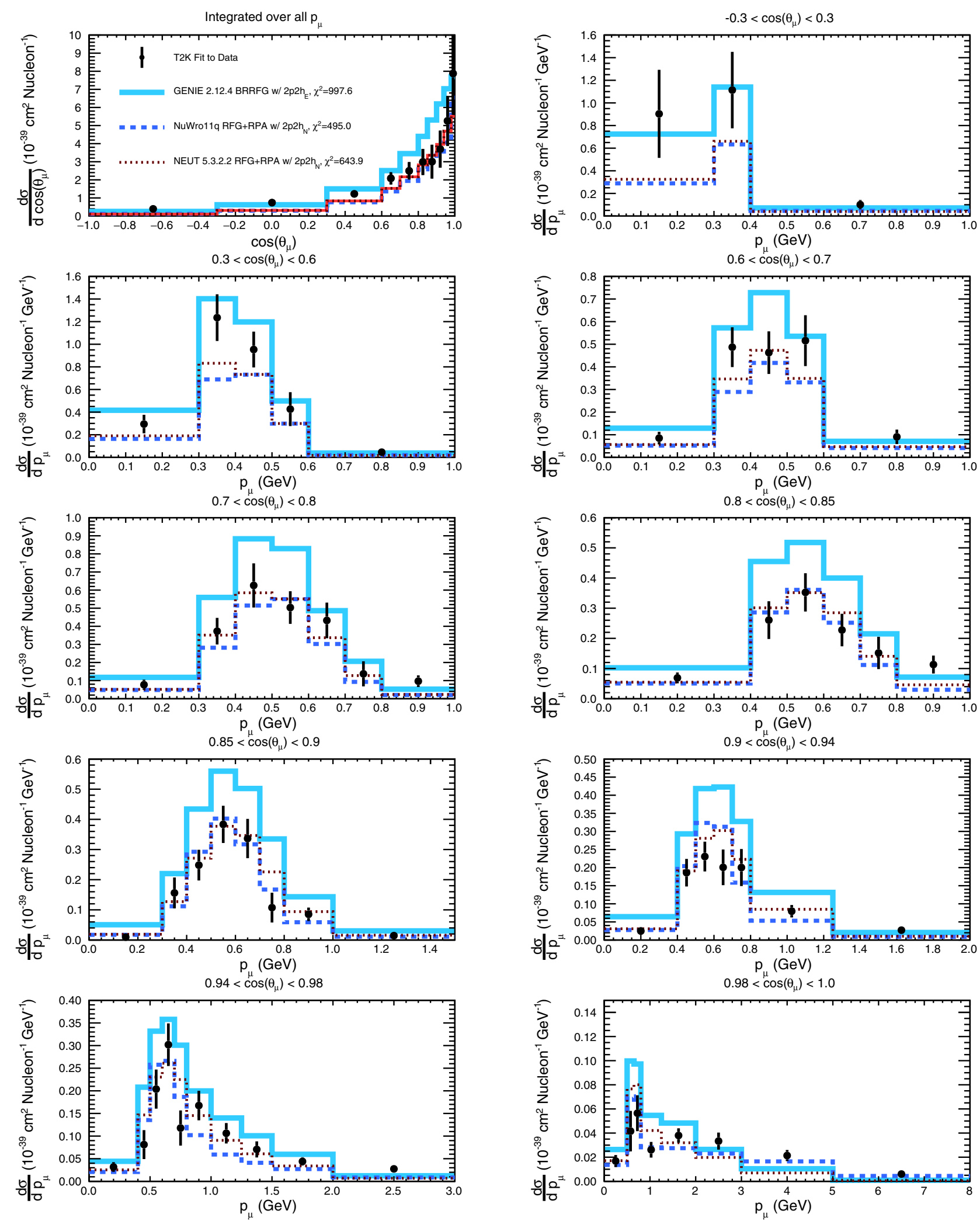

FIG. 24. The extracted differential cross section as a function of the muon kinematics in the sample without any protons (with momenta above $500 \mathrm{MeV}$ ) compared to GENIE 2.12.4, NuWro 11q and NEUT 5.3.2.2 predictions which utilize an RFG or RFG + RPA nuclear model. GENIE's RFG model also includes the empirical correction from Bodek and Ritchie (BRRFG) [81]. The NEUT and GENIE predictions shown here are similar to those used as a starting point for the T2K and NO $\nu$ A experiment's oscillation analyses, respectively. More details of these models can be found in Sec. IV A. Note that the last bin in each momentum plot is shortened for improved readability. 

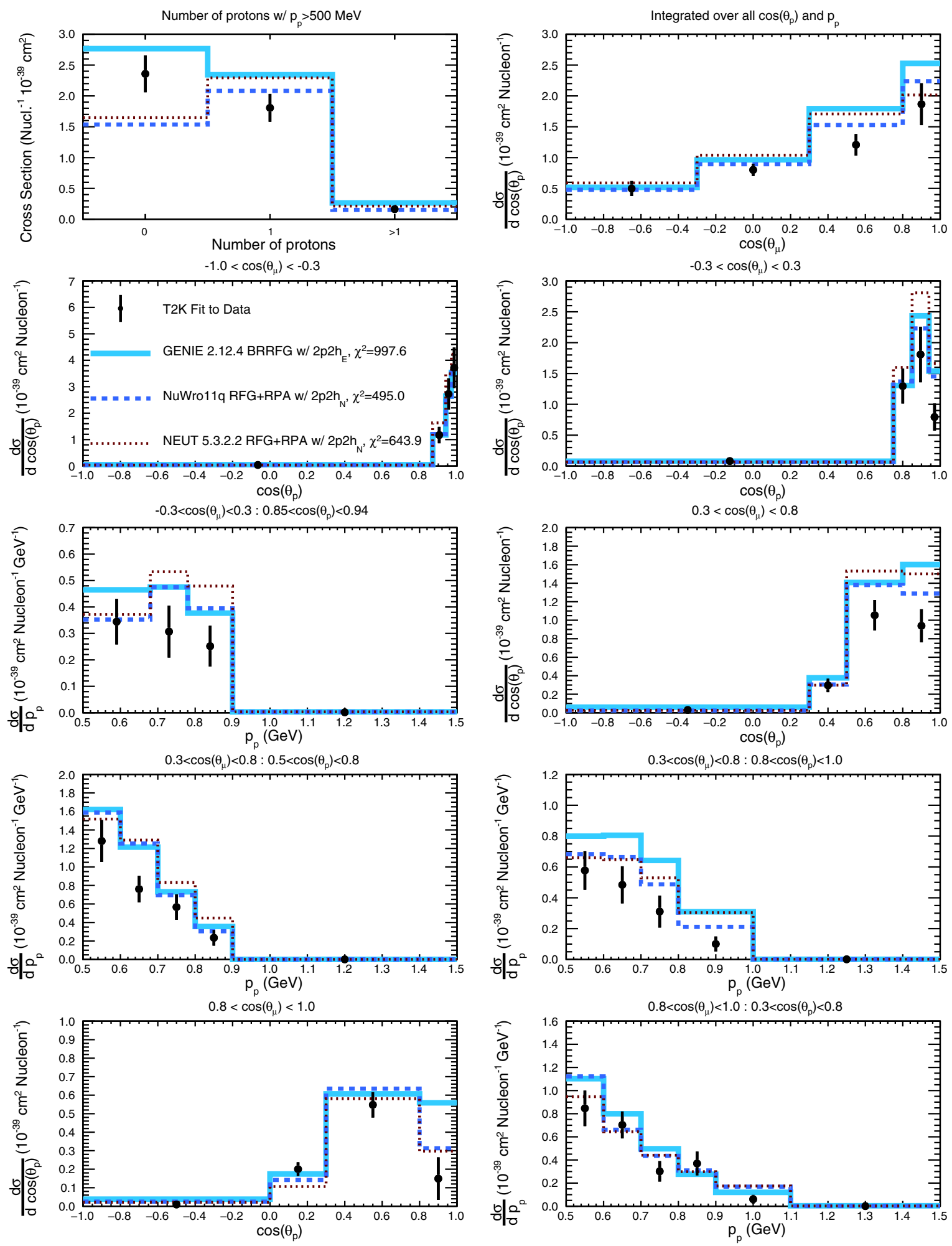

FIG. 25. The extracted differential cross section as a function of the proton multiplicity (top left) and of the proton and muon kinematics in the sample with exactly one proton (with momentum above $500 \mathrm{MeV}$ ) compared to GENIE 2.12.4, NuWro 11q and NEUT 5.3.2.2 predictions which utilize an RFG or RFG + RPA nuclear model. GENIE's RFG model also includes the empirical correction from Bodek and Ritchie (BRRFG) [81]. The NEUT and GENIE predictions shown here are similar to those used as a starting point for the $\mathrm{T} 2 \mathrm{~K}$ and $\mathrm{NO} \nu \mathrm{A}$ experiment's oscillation analyses, respectively. The $2 \mathrm{p} 2 \mathrm{~h}$ subscript indicates whether it is an implementation of the Nieves et al. model of Ref. [78] (N) or the GENIE empirical 2p2h model (E). The same models are also compared to the cross section as a function of proton multiplicity. More details of these models can be found in Sec. IV A. Note that the last bin in each momentum plot is shortened for improved readability. 
The left-hand plots of Fig. 16 show the comparison of STV with NEUT 5.3.2.2 model, demonstrating in more detail how the $2 \mathrm{p} 2 \mathrm{~h}$ contribution is clearly located in the tails of $\delta p_{\mathrm{T}}$ and $\delta \phi_{\mathrm{T}}$, where the agreement with the data is good and the $2 \mathrm{p} 2 \mathrm{~h}$ contribution seems essential. It also highlights the CCQE dominance in the bulk of the $\delta p_{\mathrm{T}}$ distribution, where the model tends to overestimate the data.

The distribution of $\delta p_{\mathrm{T}}$ beyond $400 \mathrm{MeV}$ and the shape of $\delta \alpha_{\mathrm{T}}$ are also sensitive to intra-nucleus momentum exchange such as $2 \mathrm{p} 2 \mathrm{~h}$, as already discussed, as well as FSI. As can be seen in right plots of Fig. 16, in order to bring the NEUT 5.3.2.2 model in to agreement with the data, the proton FSI strength must be increased by reducing the mean free path between reinteractions inside the nucleus by a a factor of two. Although it is challenging to draw firm conclusions from external measurements of electron-nucleus scattering, this appears to be around the maximum plausible variation based on the data [91]. Moreover, in the present semiclassical model of FSI implemented in all the simulations, the FSI mainly affect the probability of observing the outgoing proton (here defined as proton momentum above $450 \mathrm{MeV}$; see Table I) thus changing the integrated cross section. Only small modifications to the shape of the STV distributions are visible. As can be seen in Fig. 16, as the FSI strength increases, both $\delta p_{\mathrm{T}}$ and $\delta \phi_{\mathrm{T}}$ spectra become harder-with depletion and enhancement in regions of small and large imbalances, respectively-as is expected from the intranucleus momentum transfer during FSI. Nevertheless, the enhancement in this particular FSI model is much smaller than that caused by the presence of $2 \mathrm{p} 2 \mathrm{~h}$ in the region of high transverse kinematic imbalance, so it is far too small to invalidate the evidence for a $2 \mathrm{p} 2 \mathrm{~h}$ contribution.

The GENIE predictions in the left plots of Fig. 17 strongly overestimate the data in the collinear regions where the proton momentum aligns with the three-momentum transfer in the transverse plane (see $\vec{p}_{\mathrm{T}}^{\mathrm{p}}$ and $\vec{q}_{\mathrm{T}}$ in Fig. 2), i.e., in regions where $\delta p_{\mathrm{T}} \rightarrow 0, \delta \phi_{\mathrm{T}} \rightarrow 0$, and $\delta \alpha_{\mathrm{T}} \rightarrow 0$ and 180 degrees. Such overprediction originates from the elastic interaction of GENIE's widely used hA FSI model [46]. Moreover, compared to other $2 \mathrm{p} 2 \mathrm{~h}$ models, the empirical MEC model in GENIE features a much stronger enhancement in regions of large imbalances, where the overall predictions clearly overestimate the data.

The GiBUU predictions (the right plots of Fig. 17) provide an integrated cross section in good agreement with the data but it is characterized by one of the hardest $\delta p_{\mathrm{T}}$ and $\delta \phi_{\mathrm{T}}$ distributions of all the simulations, as can be seen in right plot Fig. 15, which is in disagreement with the measured shape of the observables. However, it should be noted that there is theoretical motivation to reduce GiBUU's 2p2h model strength by a factor of two [74] and an exploration of the results presented here within a more recent version of GiBUU with such reduced $2 \mathrm{p} 2 \mathrm{~h}$ has recently shown much better agreement [92].
Figure 17 and the left-hand plots of Fig. 16 also demonstrate that the contribution from RES pion-production within the STV restricted phase space is universally small relative to the $2 \mathrm{p} 2 \mathrm{~h}$ contributions (except perhaps in the final bins of $\delta p_{\mathrm{T}}$ and $\delta \phi_{\mathrm{T}}$ ). For this reason even relatively large changes in the prediction for the RES contribution to the result would not invalidate the conclusions regarding $2 \mathrm{p} 2 \mathrm{~h}$.

Finally, Figs. 18-20 show the results of the crosssection measurement as a function of the proton inferred kinematics $\left[\Delta p_{p}, \Delta \theta_{p},\left|\Delta \mathbf{p}_{p}\right|\right.$, as in Eq. (7)], compared to different models. The most precise measurements come from the region with largest statistics: $0.0<\cos \theta<0.8$, $p_{\mu}>250 \mathrm{MeV}$, which corresponds to the region of intermediate $Q^{2}$. Similarly to what was observed in the STV analysis, this region is best described by the SF model and in the high $\left|\Delta \vec{p}_{p}\right|$ tail a net preference for the presence of $2 \mathrm{p} 2 \mathrm{~h}$ contribution is visible. Such indication is independent of the strength of FSI effects, as shown in Figs. 21-23, where there is also a small preference for larger FSI.

The other regions of muon kinematics are not all consistently well described by any of the models, as can be seen by the high $\chi^{2}$ values. Depending on the kinematic region and on the observable considered, the LFG or SF may better describe the data. It is also interesting to note that both the very forward-going and low momentum muon kinematic bins suggest a multitude of regions of $\Delta p_{p}$ and $\left|\Delta \mathbf{p}_{p}\right|$ which are largely dominated by the $2 \mathrm{p} 2 \mathrm{~h}$ contribution and largely independent of FSI variation, as can be seen in figures Figs. 18-19 and 21-22, respectively. However whether the result favors these large $2 \mathrm{p} 2 \mathrm{~h}$ contributions depends on the kinematic bin: for example both the NEUT and NuWro SF and $2 \mathrm{p} 2 \mathrm{~h}$ predictions largely agree with the result in the $0.8<\cos \theta<1.0, p_{\mu}>750 \mathrm{MeV}$ bin, but are quite different in the $0.8<\cos \theta<1.0,250<p_{\mu}<750 \mathrm{MeV}$ bin. This difference is understood to stem from the substantial RES pion-production contribution to these bins, which differs considerably in NuWro and NEUT. These bins therefore offer a powerful probe of the CCnonQE contribution, but specific conclusions are difficult due to the large uncertainties in both $2 \mathrm{p} 2 \mathrm{~h}$ and nuclear effects in RES interactions, making these results complementary to the multidifferential and STV analyses that mostly have a very small RES contribution.

In Appendix A 3 a comparison to RFG models is reported which, consistently with what is observed in the STV analysis, generally gives worse agreement with the result than either LFG or SF. In particular the results clearly show that both the NEUT 5.3.2.2 RFG + RPA model and the GENIE 2.12.4 BRRFG model, which are similar to the nominal models used in recent T2K [9] and $\mathrm{NO} \nu \mathrm{A}$ [2] oscillation analyses, respectively, do not describe the result well. This conclusion is supported by also considering the poor agreement between the multidifferential results and these models seen in Appendix A 1, 
and even more so considering the aforementioned strong contention between the STV analysis results and the RFG and empirical MEC models.

\section{CONCLUSIONS}

This paper has presented the measurement with ND280 data of muon and proton multidifferential cross section, as well as their kinematic correlations, for charged-current neutrino-nucleus scattering without pion in the final state. The muon-proton correlations in the final state are measured through two new sets of variables never used before as observables in neutrino cross-section measurements: the STV and the proton inferred kinematics. The analysis selection separates events without protons, with 1 proton or with more than 1 proton in the final state above a $500 \mathrm{MeV}$ momentum threshold, thus enabling also the measurement of proton multiplicity. Particular care has been taken to minimize the model-dependence of the measurement in efficiency corrections, background subtraction and cross-section evaluation, thus enabling an unbiased and large set of model comparisons with the results. For the first time in neutrino scattering measurements the concept of data-driven regularization is introduced to achieve a result that is easy to interpret but contains minimal bias. Overall the results offer a powerful new probe of nuclear effects and that their exploration of kinematic imbalances facilitates a method of separating, at least partially, the different contributions of a $\mathrm{CC} 0 \pi$ measurement. Since prediction power of proton kinematics in neutrino-interaction simulations is still poor, it remains challenging to draw firm quantitative conclusions. Nevertheless, an extensive comparison with generator predictions has allowed interesting qualitative conclusions to be drawn. As briefly summarized below, the three analyses suggest similar conclusions.

The RFG model is able to describe only a very limited region of phase space (and only when there is no abovethreshold proton in the final state) and is categorically disfavored when considering the result in $\delta p_{\mathrm{T}}$. The LFG prediction shows slightly better agreement with data than RFG when considering interactions with above-threshold protons, especially considering the distribution of STV, but it still overestimates the soft part of the STV spectrum. A more consistent LFG implementation, such as the one in NEUT 5.4.0, gives improved results. It provides better agreement with both the STV distributions and in the region without above-threshold protons in the final state and with large muon angle, where no other model is able to describe the result. For the events with one or more above-threshold protons in the final state, the best description of the data is given by the SF model. Beyond the nucleon-nucleon correlations already included in SF, a clear requirement for a $2 \mathrm{p} 2 \mathrm{~h}$ contribution is visible in the hard tail of STV distributions $\left(\delta p_{\mathrm{T}}\right.$ and $\left.\delta \phi_{\mathrm{T}}\right)$ and of the $\left|\Delta \vec{p}_{p}\right|$ distribution. The requirement for a large $2 \mathrm{p} 2 \mathrm{~h}$ contribution to the result remains even with dramatic variations of the semiclassical FSI models available in the simulations. On the other hand, GENIE's "empirical MEC" $2 \mathrm{p} 2 \mathrm{~h}$ model appears to substantially overemphasize the hard tail of the STV. The prominent features of the STV predicted by GENIE's hA FSI model (driven by its elastic component) are in very poor agreement with the results.

The results with one proton in the final state, when compared to the SF model, suggest the need for stronger FSI effects, at the limit to what is allowed by external data of proton-nucleus scattering. The measurement of proton multiplicity can in principle disentangle $2 \mathrm{p} 2 \mathrm{~h}$ from FSI effects, with the former increasing the cross section in all bins of proton multiplicity while the latter redistributing events between different bins. Currently the primary limitation is the absence of a model able to properly describe both the events with and without above-threshold protons in the final state.

The measurement of neutrino-nucleus interactions with a pionless final state with protons clearly shows the potential to provide an even more detailed characterization of nuclear effects in neutrino-nucleus scattering in the future. To this aim, larger statistics are needed, alongside more robust predictions of outgoing proton kinematics in $2 \mathrm{p} 2 \mathrm{~h}$ and FSI models.

\section{ACKNOWLEDGMENTS}

We thank the J-PARC staff for superb accelerator performance. We thank the CERN NA61/SHINE Collaboration for providing valuable particle production data. We acknowledge the support of MEXT, Japan; NSERC (Grant No. SAPPJ2014-00031), NRC and CFI, Canada; CEA and CNRS/ IN2P3, France; DFG, Germany; INFN, Italy; National Science Centre (NCN) and Ministry of Science and Higher Education, Poland; RSF, RFBR, and MES, Russia; MINECO and ERDF funds, Spain; SNSF and SERI, Switzerland; STFC, UK; and DOE, USA. We also thank CERN for the UA1/ NOMAD magnet, DESY for the HERA-B magnet mover system, NII for SINET4, the WestGrid and SciNet consortia in Compute Canada, and GridPP in the United Kingdom. In addition, participation of individual researchers and institutions has been further supported by funds from ERC (FP7), H2020 Grant No. RISE-GA644294-JENNIFER, EU; JSPS, Japan; Royal Society, UK; the Alfred P. Sloan Foundation and the DOE Early Career program, USA.

\section{APPENDIX A: FURTHER COMPARISONS TO MODELS}

\section{Multidifferential cross-section measurement}

As discussed in Sec. IVE, this Appendix provides additional comparisons of the multidifferential analysis results to various model predictions. Figures 24 and 25 compare the results to various models that use an RFG/RFG + RPA nuclear model. Figures 26 and 27 

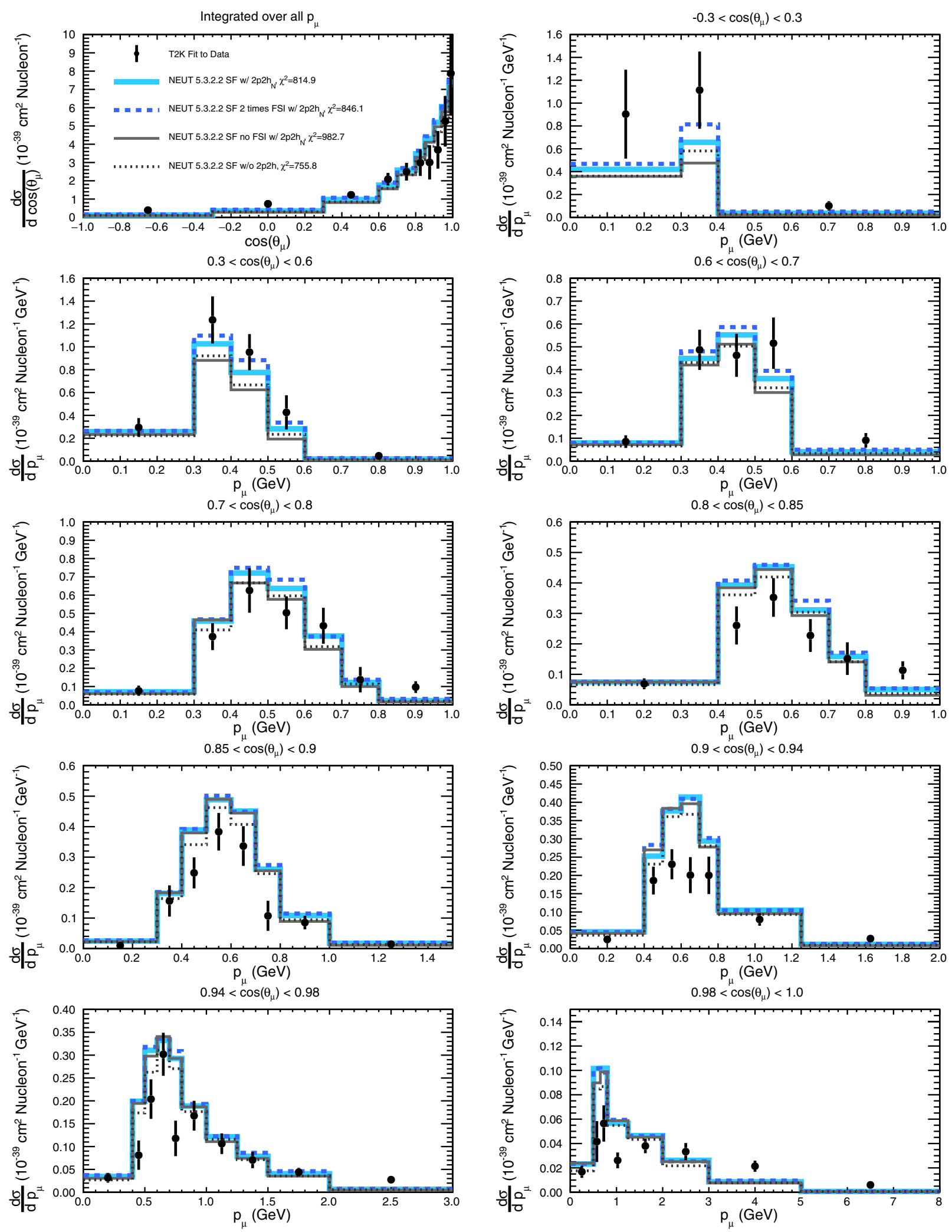

FIG. 26. The extracted differential cross section as a function of the muon kinematics in the sample without any protons (with momenta above $500 \mathrm{MeV}$ ) compared to NEUT 5.3.2.2 using the SF nuclear model with and without a $2 \mathrm{p} 2 \mathrm{~h}$ prediction and with zero or doubled FSI strength (achieved with alterations to the mean free path of FSI). $2 p 2 h_{N}$ indicates the $2 \mathrm{p} 2 \mathrm{~h}$ model is an implementation of the Nieves et al. model of Ref. [78]. More details of these models can be found in Sec. IV A. Note that the last bin in each momentum plot is shortened for improved readability. 

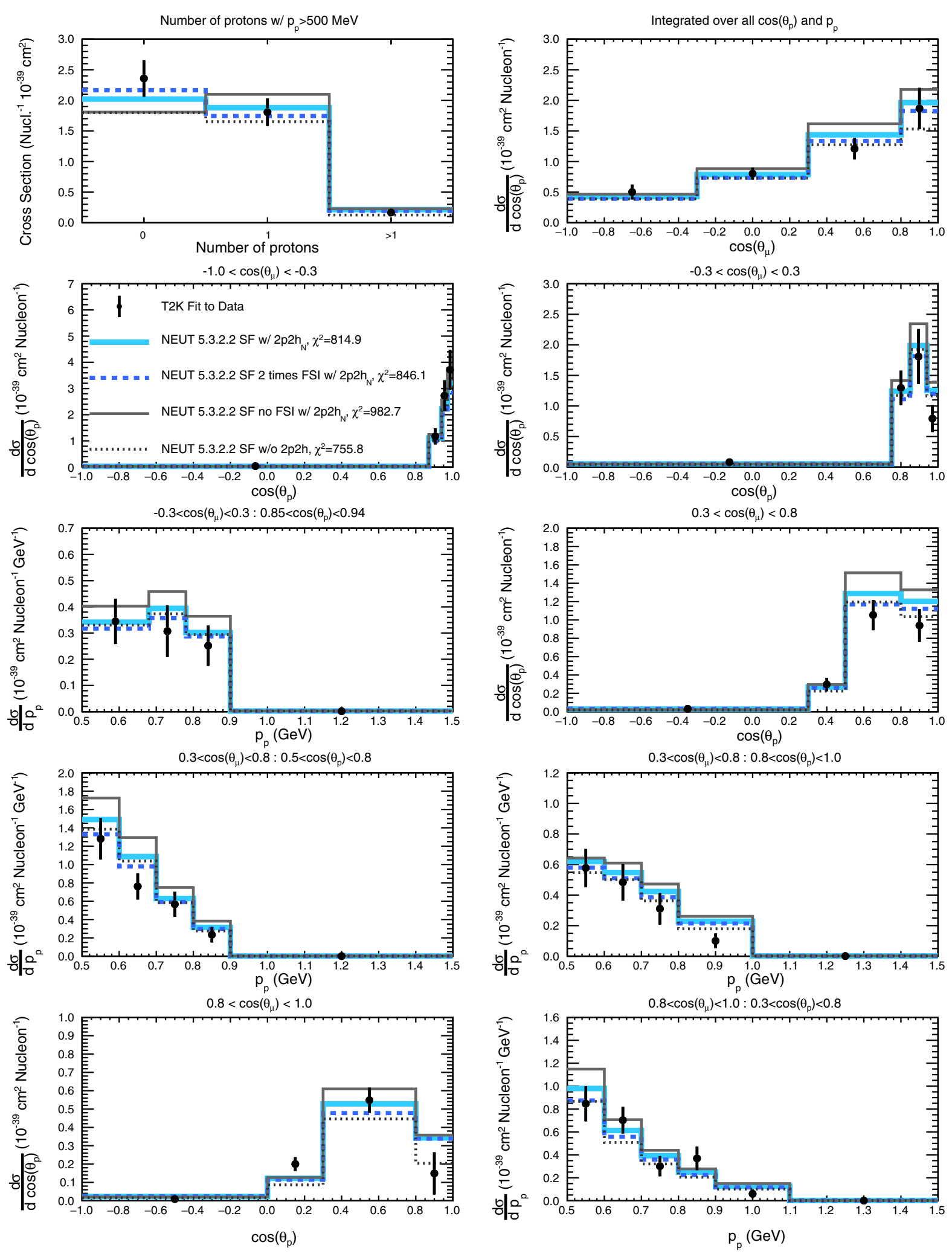

FIG. 27. The extracted differential cross section as a function of the proton multiplicity (top left) and of the proton and muon kinematics in the sample with exactly one proton (with momentum above 500 MeV) compared to NEUT 5.3.2.2 using a Benhar Spectral Function nuclear model with and without a $2 \mathrm{p} 2 \mathrm{~h}$ prediction (based on the Nieves model) and with zero or doubled FSI strength (achieved with alterations to the mean free path of FSI). The same models are also compared to the cross section as a function of proton multiplicity. $2 p 2 h_{N}$ indicates the $2 \mathrm{p} 2 \mathrm{~h}$ model is an implementation of the Nieves et al. model of Ref. [78]. More details of these models can be found in Sec. IV A. Note that the last bin in each momentum plot is shortened for improved readability. 
assess the impact of $2 \mathrm{p} 2 \mathrm{~h}$ and of FSI strength alterations on the comparison of NEUT predictions to the results.

\section{STV measurement}

As discussed in Sec. IV F, this Appendix provides additional comparisons of the single transverse analysis results to assess the impact of RPA on the data-simulation comparisons and also to identify the role of regularization in the cross-section extraction procedure. This is shown in Fig. 28 which first shows NuWro 11q predictions with and without RPA compared to the same regularized results. Alongside this, the figure also shows the predictions of
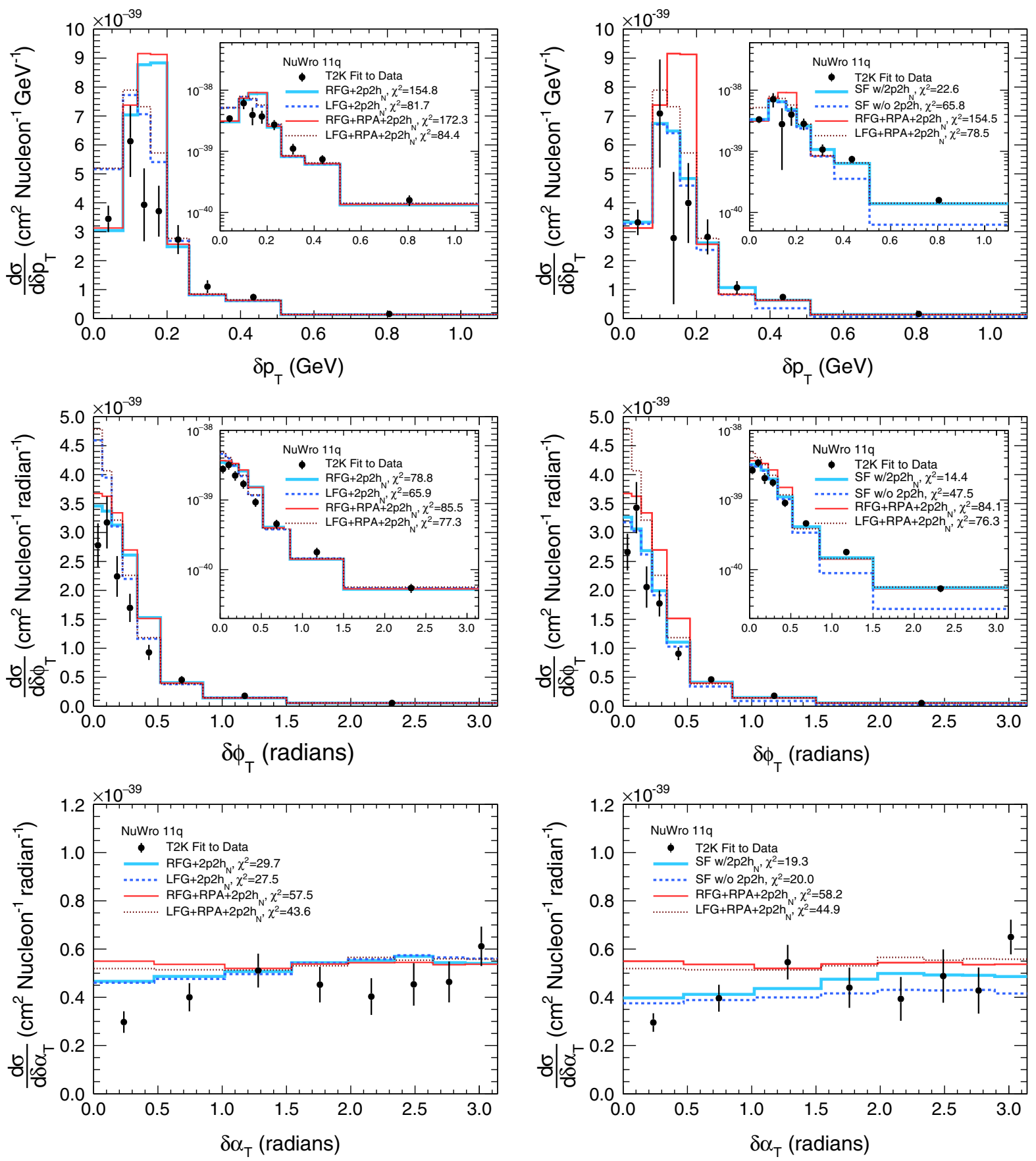

FIG. 28. The plots on the left show the extracted differential cross section as a function of the single transverse variables compared to different initial state models in the NuWro 11q simulation with and without RPA. The plots on the right show the extracted unregularized differential cross section as a function of the single transverse variables compared to different initial state models in the NuWro 11q simulation. $2 p 2 h_{N}$ indicates the Nieves et al. model of Ref. [78]. More details of these models can be found in Sec. IV A. The inlays show the same comparisons on a logarithmic scale. 


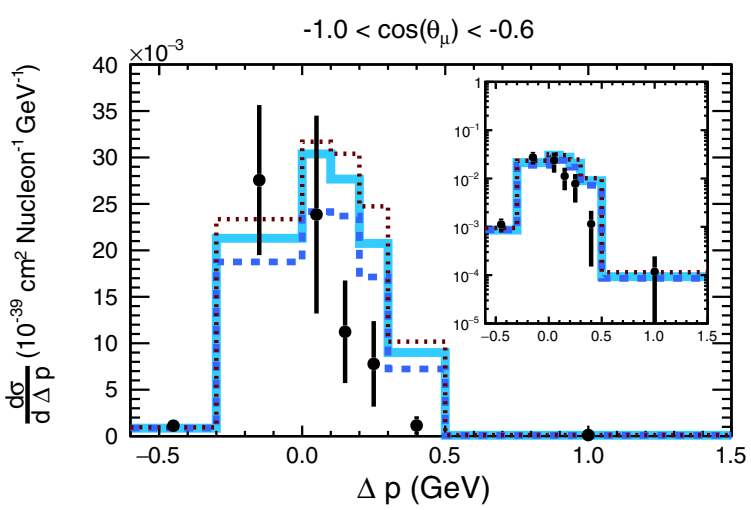

2K Unfolded
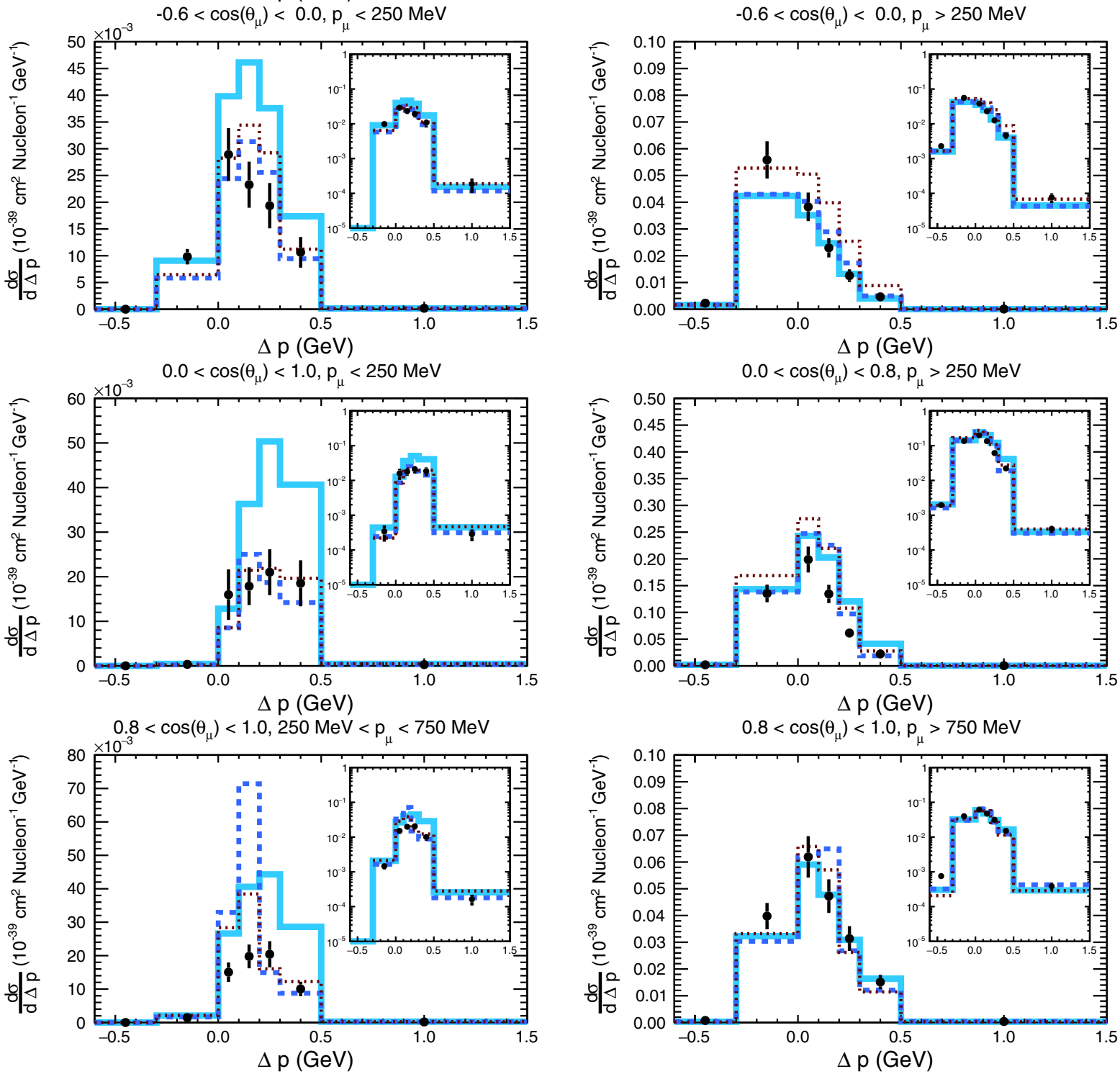

FIG. 29. The extracted differential cross section as a function of the inferred and true proton momentum difference in different muon kinematic bins, within a restricted proton kinematic phase space, compared to GENIE 2.12.4, NuWro 11q and NEUT 5.3.2.2 predictions which utilize an RFG or RFG + RPA nuclear model. GENIE's RFG model also includes the empirical correction from Bodek and Ritchie (BRRFG) [81]. The NEUT and GENIE predictions shown here are similar to those used as a starting point for the T2K and $\mathrm{NO} \nu \mathrm{A}$ experiment's oscillation analyses, respectively. More details of these models can be found in Sec. IV A. The $2 \mathrm{p} 2 \mathrm{~h}$ subscript indicates whether it is an implementation of the Nieves et al. model of Ref. [78] (N) or the GENIE empirical 2p2h model (E). More details of these models can be found in Sec. IV A. Note that the first and last bin in each plot is shortened for improved readability. The inlays show the same comparisons on a logarithmic scale. 


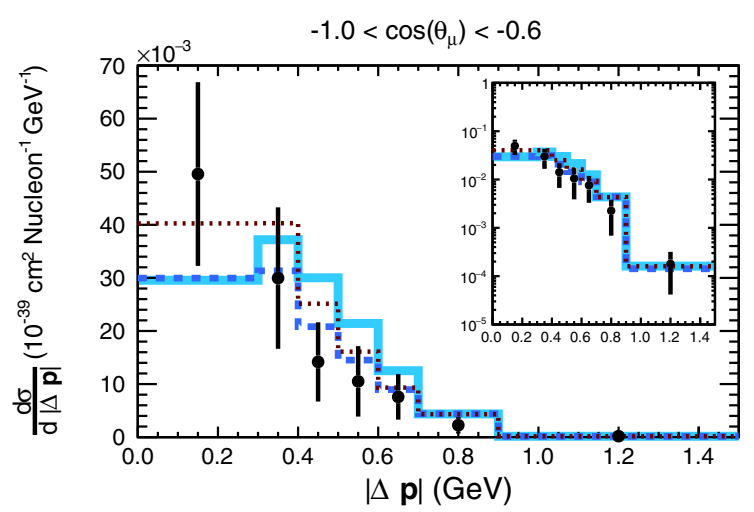

2K Unfolded
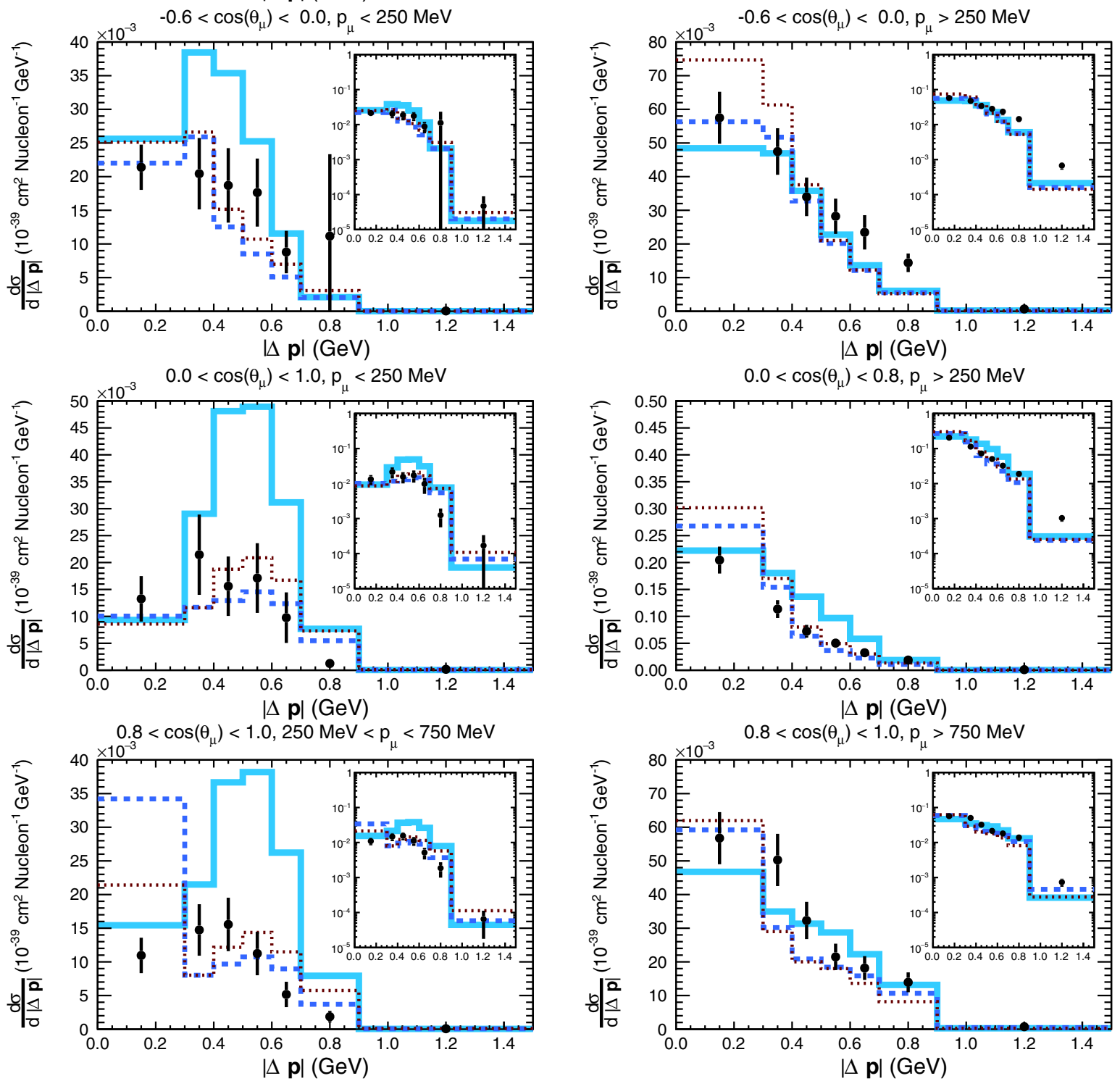

FIG. 30. The extracted differential cross section as a function of the modulus of the inferred and true proton three-momentum difference in different muon kinematic bins, within a restricted proton kinematic phase space, compared to GENIE 2.12.4, NuWro 11q and NEUT 5.3.2.2 predictions which utilize an RFG or RFG + RPA nuclear model. GENIE's RFG model also includes the empirical correction from Bodek and Ritchie (BRRFG) [81]. The NEUT and GENIE predictions shown here are similar to those used as a starting point for the $\mathrm{T} 2 \mathrm{~K}$ and $\mathrm{NO} \nu \mathrm{A}$ experiment's oscillation analyses, respectively. The $2 \mathrm{p} 2 \mathrm{~h}$ subscript indicates whether it is an implementation of the Nieves et al. model of Ref. [78] (N) or the GENIE empirical 2p2h model (E). More details of these models can be found in Sec. IVA. Note that the last bin in each plot is shortened for improved readability. The inlays show the same comparisons on a logarithmic scale. 

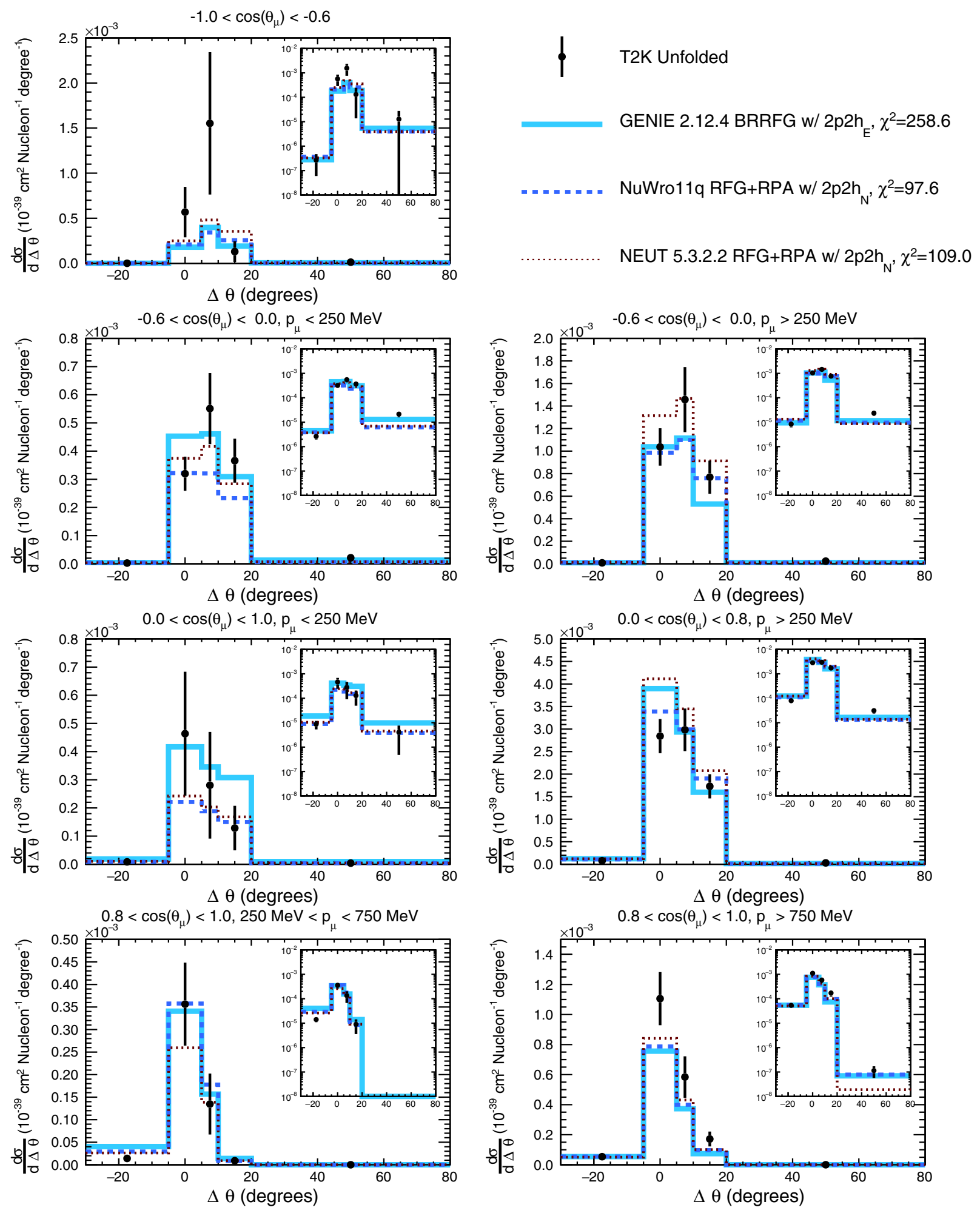

FIG. 31. The extracted differential cross section as a function of the inferred and true proton outgoing-angle difference in different muon kinematic bins, within a restricted proton kinematic phase space, compared to GENIE 2.12.4, NuWro 11q and NEUT 5.3.2.2 predictions which utilize an RFG or RFG + RPA nuclear model. GENIE's RFG model also includes the empirical correction from Bodek and Ritchie (BRRFG) [81]. The NEUT and GENIE predictions shown here are similar to those used as a starting point for the $\mathrm{T} 2 \mathrm{~K}$ and $\mathrm{NO} \nu \mathrm{A}$ experiment's oscillation analyses, respectively. The $2 \mathrm{p} 2 \mathrm{~h}$ subscript indicates whether it is an implementation of the Nieves et al. model of Ref. [78] (N) or the GENIE empirical 2p2h model (E). More details of these models can be found in Sec. IV A. Note that the first and last bin in each plot is shortened for improved readability. The inlays show the same comparisons on a logarithmic scale. 
different nuclear models, already shown in Fig. 15, compared to the unregularized results. In this final figure, it should be noted that, while the nominal result is different than the regularized result presented in Sec. IV F, the physics conclusions remain the same with a similar goodness of fit.

\section{Inferred proton kinematics measurement}

As discussed in Sec. IV G, this Appendix provides additional comparisons of the proton inferred kinematics analysis results to RFG nuclear models. This is shown in Fig. 29-31, which compare the extracted results to RFG predictions from NEUT 5.3.2.2, NuWro $11 \mathrm{q}$ and GENIE 2.12.4.

\section{APPENDIX B: $\chi^{2}$ COMPARISONS TO THE STV RESULTS WITH AND WITHOUT REGULARIZATION}

As discussed in Sec. IV D 1, the regularization of cross-section extraction methods allows results that are easy to interpret at the cost of some bias. Although the regularized STV results presented in Sec. IV F minimize this using a data-driven method, the unregularized results are also produced to guarantee no unfolding bias. To demonstrate that the application of regularization does not alter the physical interpretation of the results, Tables IX-XI show a summary of the $\chi^{2}$ agreement between the various models considered in Sec. IV F and

TABLE IX. The full and shape-only $\chi^{2}$ comparisons to the $\delta p_{T}$ result with nominal and no regularization. The table is ordered by the size of the no-regularization shape-only $\chi^{2}$. More details of these models can be found in Sec. IVA.

\begin{tabular}{|c|c|c|c|c|}
\hline \multirow[b]{2}{*}{ Generator } & \multicolumn{2}{|c|}{ Full } & \multicolumn{2}{|c|}{ Shape Only } \\
\hline & No Reg. & Nom. Reg. & No Reg. & Nom. Reg. \\
\hline NEUT $5.4 .0\left(\mathrm{LFG}_{N}+2 p 2 h_{N}\right)$ & 31.6 & 30.4 & 3.38 & 2.60 \\
\hline NEUT 5.3.2.2 $\left(\mathrm{SF}+2 p 2 h_{N}+2 \times \mathrm{FSI}\right)$ & 15.9 & 14.8 & 11.0 & 10.1 \\
\hline NEUT 5.3.2.2 $\left(\mathrm{SF}+2 p 2 h_{N}\right)$ & 31.9 & 30.3 & 16.6 & 15.5 \\
\hline NuWro 11q $\left(\mathrm{SF}+2 p 2 h_{N}\right)$ & 22.6 & 23.1 & 16.8 & 15.6 \\
\hline NuWro 11q $\left(\mathrm{LFG}+2 p 2 h_{N}\right)$ & 81.5 & 81.7 & 39.0 & 15.6 \\
\hline NuWro $11 \mathrm{q}\left(\mathrm{LFG}+\mathrm{RPA}+2 p 2 h_{N}\right)$ & 78.5 & 84.4 & 39.9 & 36.3 \\
\hline NEUT 5.3.2.2 $\left(\mathrm{SF}+2 p 2 h_{N}+\right.$ No FSI $)$ & 114 & 112 & 42.9 & 41.4 \\
\hline GENIE $2.12 .4\left(\mathrm{RFG}+2 p 2 h_{E}\right)$ & 92.9 & 92.4 & 47.9 & 47.7 \\
\hline NuWro 11q (SF w/o 2p2h) & 65.8 & 68.7 & 55.4 & 54.8 \\
\hline NEUT 5.3.2.2 (SF w/o 2p2h) & 93.3 & 91.5 & 61.2 & 59.6 \\
\hline GiBUU $2016\left(\mathrm{LFG}+2 p 2 h_{G}\right)$ & 77.0 & 78.9 & 66.1 & 59.6 \\
\hline NuWro $11 q\left(\mathrm{RFG}+2 p 2 h_{N}\right)$ & 150 & 155 & 67.2 & 69.0 \\
\hline NuWro 11q $\left(\mathrm{RFG}+\mathrm{RPA}+2 p 2 h_{N}\right)$ & 155 & 172 & 68.6 & 70.4 \\
\hline GENIE 2.12.4 (RFG w/o 2p2h) & 94.6 & 97.8 & 74.1 & 76.2 \\
\hline
\end{tabular}

TABLE X. The full and shape-only $\chi^{2}$ comparisons to the $\delta \phi_{T}$ result with nominal and no regularization. The table is ordered by the size of the no-regularization shape-only $\chi^{2}$. More details of these models can be found in Sec. IVA.

\begin{tabular}{|c|c|c|c|c|}
\hline \multirow[b]{2}{*}{ Generator } & \multicolumn{2}{|c|}{ Full } & \multicolumn{2}{|c|}{ Shape Only } \\
\hline & No Reg. & Nom. Reg. & No Reg. & Nom. Reg. \\
\hline NEUT $5.4 .0\left(\mathrm{LFG}_{N}+2 p 2 h_{N}\right)$ & 39.0 & 36.7 & 7.55 & 6.40 \\
\hline NEUT 5.3.2.2 $\left(\mathrm{SF}+2 p 2 h_{N}+2 \times \mathrm{FSI}\right)$ & 9.95 & 8.70 & 7.71 & 6.57 \\
\hline NEUT 5.3.2.2 $\left(\mathrm{SF}+2 p 2 h_{N}\right)$ & 18.4 & 17.0 & 9.59 & 8.45 \\
\hline NuWro 11q $\left(\mathrm{SF}+2 p 2 h_{N}\right)$ & 14.4 & 13,5 & 10.8 & 9.70 \\
\hline NuWro 11q $\left(\mathrm{LFG}+2 p 2 h_{N}\right)$ & 66.8 & 65.9 & 29.7.0 & 29.0 \\
\hline NEUT 5.3.2.2 $\left(\mathrm{SF}+2 p 2 h_{N}+\right.$ No FSI $)$ & 81.5 & 81.4 & 30.5 & 30.1 \\
\hline NuWro 11q $\left(\mathrm{LFG}+\mathrm{RPA}+2 p 2 h_{N}\right)$ & 76.3 & 77.3 & 32.1 & 31.3 \\
\hline NuWro 11q $\left(\mathrm{RFG}+\mathrm{RPA}+2 p 2 h_{N}\right)$ & 84.7 & 85.5 & 40.1 & 39.4 \\
\hline NuWro 11q (SF w/o 2p2h) & 47.5 & 48.9 & 42.1 & 42.3 \\
\hline NuWro $11 \mathrm{q}\left(\mathrm{RFG}+2 p 2 h_{N}\right)$ & 79.3 & 78.8 & 42.6 & 42.0 \\
\hline NEUT 5.3.2.2 (SF w/o 2p2h) & 60.6 & 61.0 & 43.7 & 43.8 \\
\hline GiBUU $2016\left(\mathrm{LFG}+2 p 2 h_{G}\right)$ & 43.4 & 44.1 & 45.6 & 46.2 \\
\hline GENIE $2.12 .4\left(\mathrm{RFG}+2 p 2 h_{E}\right)$ & 208 & 211 & 114 & 115 \\
\hline GENIE 2.12.4 (RFG w/o 2p2h) & 192 & 193 & 128 & 128 \\
\hline
\end{tabular}


TABLE XI. The full and shape-only $\chi^{2}$ comparisons to the $\delta \alpha_{T}$ result with nominal and no regularization. The table is ordered by the size of the no-regularization shape-only $\chi^{2}$. More details of these models can be found in Sec. IV A.

\begin{tabular}{|c|c|c|c|c|}
\hline \multirow[b]{2}{*}{ Generator } & \multicolumn{2}{|c|}{ Full } & \multicolumn{2}{|c|}{ Shape only } \\
\hline & No reg. & Nom. reg. & No reg. & Nom. reg. \\
\hline NEUT 5.3.2.2 $\left(\mathrm{SF}+2 p 2 h_{N}+2 \times\right.$ FSI $)$ & 17.7 & 15.8 & 16.3 & 14.2 \\
\hline NuWro 11q $\left(\mathrm{SF}+2 p 2 h_{N}\right)$ & 19.3 & 18.0 & 18.6 & 16.6 \\
\hline NEUT 5.3.2.2 $\left(\mathrm{SF}+2 p 2 h_{N}\right)$ & 24.8 & 23.0 & 18.8 & 16.8 \\
\hline NuWro 11q $\left(\mathrm{LFG}+2 p 2 h_{N}\right)$ & 29.6 & 27.5 & 19.0 & 16.9 \\
\hline NuWro 11q $\left(\mathrm{RFG}+2 p 2 h_{N}\right)$ & 31.6 & 29.7 & 20.7 & 18.7 \\
\hline NEUT 5.3.2.2 (SF w/o 2p2h) & 21.0 & 19.5 & 21.7 & 19.6 \\
\hline NEUT $5.4 .0\left(\mathrm{LFG}_{N}+2 p 2 h_{N}\right)$ & 63.0 & 60.7 & 22.8 & 20.8 \\
\hline NuWro 11q (SF w/o 2p2h) & 20.0 & 18.9 & 23.4 & 21.4 \\
\hline NEUT 5.3.2.2 (SF $+2 p 2 h_{N}+$ No FSI $)$ & 49.9 & 48.2 & 28.3 & 26.3 \\
\hline NuWro 11q $\left(\mathrm{LFG}+\mathrm{RPA}+2 p 2 h_{N}\right)$ & 44.9 & 43.6 & 28.6 & 26.3 \\
\hline GiBUU $2016\left(\mathrm{LFG}+2 p 2 h_{G}\right)$ & 41.3 & 40.2 & 35.5 & 33.7 \\
\hline NuWro 11q $\left(\mathrm{RFG}+\mathrm{RPA}+2 p 2 h_{N}\right)$ & 58.2 & 57.5 & 38.1 & 35.8 \\
\hline GENIE $2.12 .4\left(\mathrm{RFG}+2 p 2 h_{E}\right)$ & 88.5 & 90.2 & 40.1 & 39.6 \\
\hline GENIE 2.12.4 (RFG w/o 2p2h) & 38.6 & 72.0 & 62.6 & 64.1 \\
\hline
\end{tabular}

both the regularized and the unregularized results. The shape-only $\chi^{2}$ is also shown for each model as this does not suffer from Peelle's Pertinent Puzzle discussed in Sec. V and so may be the most useful quantitative metric for comparing model agreement. This shape-only $\chi^{2}$ is formed by numerically decomposing the full covariance matrix into a shape and normalization component. Occasionally it was found that it was necessary to add a small component to the diagonal of the shape-only covariance matrix to make the matrix invertible, thereby slightly overestimating the error (this is responsible for the two instances of seeing a shape-only $\chi^{2}$ greater than the full $\chi^{2}$ in Table XI).

Overall the $\chi^{2}$ tables show very little difference between the regularized and unregularized result, demonstrating that the cautious data-driven choice of regularization strength means that only a very small bias is added. The tables echo the conclusions drawn in Sec. V: a 2p2h contribution and a non-RFG nuclear model is shown to be a hallmark of the lower $\chi^{2}$ in the comparisons.
[1] Y. Fukuda et al. (Super-Kamiokande Collaboration), Phys. Rev. Lett. 81, 1562 (1998).

[2] Q. R. Ahmad et al. (SNO Collaboration), Phys. Rev. Lett. 87, 071301 (2001).

[3] Q. R. Ahmad et al. (SNO Collaboration), Phys. Rev. Lett. 89, 011301 (2002).

[4] T. Araki et al. (KamLAND Collaboration), Phys. Rev. Lett. 94, 081801 (2005).

[5] E. Aliu et al. (K2K Collaboration), Phys. Rev. Lett. 94, 081802 (2005).

[6] D. G. Michael et al. (MINOS Collaboration), Phys. Rev. Lett. 97, 191801 (2006).

[7] K. Abe et al. (T2K Collaboration), Phys. Rev. Lett. 107, 041801 (2011).

[8] F. P. An et al. (Daya Bay Collaboration), Phys. Rev. Lett. 108, 171803 (2012).

[9] K. Abe et al. (T2K Collaboration), Phys. Rev. D 96, 092006 (2017).

[10] P. Adamson et al. (NOvA Collaboration), Phys. Rev. Lett. 118, 231801 (2017).
[11] K. Abe et al. (Hyper-Kamiokande Proto-Collaboration), Prog. Theor. Exp. Phys. 2015, 53C02 (2015).

[12] R. Acciarri et al. (DUNE Collaboration), arXiv:1512.06148.

[13] L. Alvarez-Ruso et al., Prog. Part. Nucl. Phys. 100, 1 (2018).

[14] K. Abe et al. (T2K Collaboration), Nucl. Instrum. Methods Phys. Res., Sect. A 659, 106 (2011).

[15] P. Adamson et al. (NOvA Collaboration), Phys. Rev. Lett. 116, 151806 (2016).

[16] K. Abe et al. (Hyper-Kamiokande Working Group), arXiv:1412.4673.

[17] A. M. Ankowski and J. T. Sobczyk, Phys. Rev. C 74, 054316 (2006).

[18] R. Gran et al. (K2K Collaboration), Phys. Rev. D 74, 052002 (2006).

[19] A. A. Aguilar-Arevalo et al. (MiniBooNE Collaboration), Phys. Rev. D 81, 092005 (2010).

[20] S. K. Singh and E. Oset, Nucl. Phys. A542, 587 (1992).

[21] A. Gil, J. Nieves, and E. Oset, Nucl. Phys. A627, 543 (1997). 
[22] J. Nieves, J. E. Amaro, and M. Valverde, Phys. Rev. C 70, 055503 (2004); 72, 019902(E) (2005).

[23] J. Nieves, M. Valverde, and M. J. Vicente Vacas, Phys. Rev. C 73, 025504 (2006).

[24] M. Martini, M. Ericson, G. Chanfray, and J. Marteau, Phys. Rev. C 80, 065501 (2009).

[25] O. Benhar, A. Fabrocini, S. Fantoni, and I. Sick, Nucl. Phys. A579, 493 (1994).

[26] O. Benhar, N. Farina, H. Nakamura, M. Sakuda, and R. Seki, Phys. Rev. D 72, 053005 (2005).

[27] O. Benhar, D. day, and I. Sick, Rev. Mod. Phys. 80, 189 (2008).

[28] A. M. Ankowski, O. Benhar, and N. Farina, Phys. Rev. D 82, 013002 (2010).

[29] J. Delorme and M. Ericson, Phys. Lett. 156B, 263 (1985).

[30] J. Marteau, J. Delorme, and M. Ericson, Nucl. Instrum. Methods Phys. Res., Sect. A 451, 76 (2000).

[31] M. Martini, M. Ericson, G. Chanfray, and J. Marteau, Phys. Rev. C 81, 045502 (2010).

[32] J. Nieves, I. Ruiz Simo, and M. J. Vicente Vacas, Phys. Rev. C 83, 045501 (2011).

[33] J. Nieves, I. Ruiz Simo, and M. J. Vicente Vacas, Phys. Lett. B 707, 72 (2012).

[34] M. Martini, M. Ericson, and G. Chanfray, Phys. Rev. C 84, 055502 (2011).

[35] V. Lyubushkin et al. (NOMAD Collaboration), Eur. Phys. J. C 63, 355 (2009).

[36] A. A. Aguilar-Arevalo et al. (MiniBooNE Collaboration), Phys. Rev. D 88, 032001 (2013).

[37] L. Fields et al. (MINERvA Collaboration), Phys. Rev. Lett. 111, 022501 (2013).

[38] G. A. Fiorentini et al. (MINERvA Collaboration), Phys. Rev. Lett. 111, 022502 (2013).

[39] T. Walton et al. (MINERvA Collaboration), Phys. Rev. D 91, 071301 (2015).

[40] J. Wolcott et al. (MINERvA Collaboration), Phys. Rev. Lett. 116, 081802 (2016).

[41] M. Betancourt et al. (MINERvA Collaboration), Phys. Rev. Lett. 119, 082001 (2017).

[42] K. Abe et al. (T2K Collaboration), Phys. Rev. D 92, 112003 (2015).

[43] K. Abe et al. (T2K Collaboration), Phys. Rev. D 91, 112002 (2015).

[44] K. Abe et al. (T2K Collaboration), Phys. Rev. D 93, 112012 (2016).

[45] R. Acciarri et al. (ArgoNeuT Collaboration), Phys. Rev. D 90, 012008 (2014).

[46] X. G. Lu, L. Pickering, S. Dolan, G. Barr, D. Coplowe, Y. Uchida, D. Wark, M. O. Wascko, A. Weber, and T. Yuan, Phys. Rev. C 94, 015503 (2016).

[47] K. Abe et al. (T2K Collaboration), Phys. Rev. D 87, 012001 (2013).

[48] K. Abe et al., Nucl. Instrum. Methods Phys. Res., Sect. A 694, 211 (2012).

[49] S. Assylbekov et al., Nucl. Instrum. Methods Phys. Res., Sect. A 686, 48 (2012).

[50] D. Allan et al., J. Instrum. 8, P10019 (2013).

[51] S. Aoki et al., Nucl. Instrum. Methods Phys. Res., Sect. A 698, 135 (2013).
[52] N. Abgrall et al. (T2K ND280 TPC Collaboration), Nucl. Instrum. Methods Phys. Res., Sect. A 637, 25 (2011).

[53] P. A. Amaudruz et al. (T2K ND280 FGD Collaboration), Nucl. Instrum. Methods Phys. Res., Sect. A 696, 1 (2012).

[54] X. G. Lu et al. (MINERvA Collaboration), arXiv:1805 .05486 [J. High Energy Phys. (to be published)].

[55] L. Pickering, J. Phys. Soc. Jpn. Conf. Proc. 12, 010032 (2016).

[56] G. D’Agostini, Nucl. Instrum. Methods Phys. Res., Sect A 362, 487 (1995).

[57] G. D'Agostini, arXiv:1010.0632.

[58] S. Dolan, Ph.D. thesis, T2K-THESIS-084, 2017.

[59] A. Ferrari, P. R. Sala, A. Fasso, and J. Ranft, CERN \& INFN, Milan, SLAC, Siegen U., Report Nos. CERN-2005010, SLAC-R-773, INFN-TC-05-11, (2005).

[60] T. Bohlen, F. Cerutti, M. Chin, A. Fasso, A. Ferrari, P. Ortega, A. Mairani, P. Sala, G. Smirnov, and V. Vlachoudis, Nucl. Data Sheets 120, 211 (2014).

[61] R. Brun, F. Carminati, and S. Giani, CERN, Report no. CERN-W5013 (1994).

[62] C. Zeitnitz and T. A. Gabriel, Proc. of International Conference on Calorimetry in High Energy Physics (World Scientific, 1993), p. 394.

[63] N. Abgrall et al. (NA61/SHINE Collaboration), Phys. Rev. C 84, 034604 (2011).

[64] N. Abgrall et al. (NA61/SHINE Collaboration), Phys. Rev. C 85, 035210 (2012).

[65] N. Abgrall et al. (NA61/SHINE Collaboration), Eur. Phys. J. C 76, 84 (2016).

[66] T. Eichten et al., Nucl. Phys. B44 (1972).

[67] J. V. Allaby et al., Technical Report No. 70-12, CERN, 1970).

[68] I. Chemakin et al. (E910 Collaboration), Phys. Rev. C 77, 015209 (2008).

[69] Y. Hayato, Nucl. Phys. B, Proc. Suppl. 112, 171 (2002).

[70] Y. Hayato, Acta Phys. Pol. B 40, 2477 (2009).

[71] S. Agostinelli et al. (GEANT4 Collaboration), Nucl. Instrum. Methods Phys. Res., Sect. A 506, 250 (2003).

[72] C. Andreopoulos, A. Bell, D. Bhattacharya, F. Cavanna, J. Dobson et al., Nucl. Instrum. Methods Phys. Res., Sect. A 614, 87 (2010).

[73] T. Golan, C. Juszczak, and J. T. Sobczyk, Phys. Rev. C 86, 015505 (2012).

[74] K. Gallmeister, U. Mosel, and J. Weil, Phys. Rev. C 94, 035502 (2016).

[75] C. H. Llewellyn Smith, Phys. Rep. 3, 261 (1972).

[76] O. Benhar, A. Fabrocini, S. Fantoni, and I. Sick, Nucl. Phys. A579, 193 (1994).

[77] D. Rein and L. M. Sehgal, Ann. Phys. (N.Y.) 133, 79 (1981).

[78] J. Nieves, I. R. Simo, and M. V. Vacas, Phys. Lett. B 707, 72 (2012).

[79] M. Gluck, E. Reya, and A. Vogt, Eur. Phys. J. C 5, 461 (1998).

[80] A. Bodek and U. K. Yang, AIP Conf. Proc. 670, 110 (2003).

[81] A. Bodek and J. L. Ritchie, Phys. Rev. D 24, 1400 (1981).

[82] O. Buss, T. Gaitanos, K. Gallmeister, H. van Hees, M. Kaskulov, O. Lalakulich, A. B. Larionov, T. Leitner, J. Weil, and U. Mosel, Phys. Rep. 512, 1 (2012). 
[83] T. Leitner, L. Alvarez-Ruso, and U. Mosel, Phys. Rev. C 73, 065502 (2006).

[84] E. Hernndez, J. Nieves, and M. J. Vicente Vacas, Phys. Rev. D 87, 113009 (2013).

[85] P. Stowell et al., J. Instrum. 12, P01016 (2017).

[86] A. Higuera et al. (MINERvA Collaboration), Phys. Rev. Lett. 113, 261802 (2014).

[87] M. Kuusela and P. B. Stark, arXiv:1512.00905.

[88] P. C. Hansen, SIAM Rev. 34, 561 (1992).
[89] P. Adamson et al. (NOvA Collaboration), Phys. Rev. Lett. 118, 151802 (2017).

[90] R. W. Peelle, Peelle's Pertinent Puzzle (Oak Ridge National Laboratory, Oakridge, 1987).

[91] D. Dutta, K. Hafidi, and M. Strikman, Prog. Part. Nucl. Phys. 69, 1 (2013).

[92] S. Dolan, U. Mosel, K. Gallmeister, L. Pickering, and S. Bolognesi, arXiv:1804.09488. 